\title{
19. RADIOLARIANS FROM SITES 434, 435, AND 436, NORTHWEST PACIFIC, LEG 56, DEEP SEA DRILLING PROJECT
}

\author{
Toyosaburo Sakai, Department of Geology, Utsunomiya University, Utsunomiya, Japan
}

\section{INTRODUCTION}

During Leg 56 of the Deep Sea Drilling Project, six holes were drilled at three sites near the Japan Trench (Table 1). Sites 434 and 435 are located on the landward wall of the trench and Site 436 on the crest of an outer rise. At all of the sites, Neogene radiolarians were consistently recovered, and the lowermost two cores of Hole 436 contained Cretaceous radiolarians as well.

Of the rich and well-preserved Neogene radiolarians recovered during this cruise, this chapter discusses only those of biostratigraphic importance. It consists of a tabulation of occurrence-abundance, a radiolarian event list, and a range chart. In addition, it includes information suggesting certain phylogenetic relationships and a brief discussion of the correlation between the zones used in this report and those established in Japanese land sequences. Because of minor recovery and the poor state of preservation of Cretaceous radiolarians, only a few Cretaceous species are discussed.

\section{METHOD OF STUDY}

Except for a chert from Cores 41 and 42 of Site 436, all samples were prepared as follows. Sediments were disaggregated by boiling in a solution of sodium hexametaphosphate and hydrogen peroxide for about 30 minutes. Next they were wet-sieved through a $63-\mu$ mesh screen and the remaining coarse fraction dried. This process was repeated until clay materials were removed from radiolarian skeletons. Then a dried sample was strewn on a glass slide $(76 \mathrm{~mm} \times 26 \mathrm{~mm})$ which had been thinly painted with a solution of gum tragacanth nearly the same width as that of a cover glass $(40 \mathrm{~mm} \times$ $22 \mathrm{~mm}$ ) and the gum moistened with aqueous vapor to fix the radiolarians on the slide. When the gum was dry, the radiolarians were removed by turning the slide over and patting it gently. Next a drop of xylene and 0.4 to $0.5 \mathrm{ml}$ of Caedax were put on the slide. The slide was

TABLE 1

\begin{tabular}{|c|c|c|c|}
\hline Iole & $\begin{array}{l}\text { Latitude } \\
\text { (N) }\end{array}$ & $\begin{array}{l}\text { Longitude } \\
\text { (E) }\end{array}$ & $\begin{array}{l}\text { Water } \\
\text { Depth } \\
\text { (m) }\end{array}$ \\
\hline & $39^{\circ} 44.76^{\prime}$ & $144^{\circ} 06.12^{\prime}$ & 5986 \\
\hline $34 \mathrm{~A}$ & $39^{\circ} 44.76^{\prime}$ & $144^{\circ} 06.12^{\prime}$ & 5986 \\
\hline $34 \mathrm{~B}$ & $39^{\circ} 44.87^{\prime}$ & $144^{\circ} 06.08^{\prime}$ & 5986 \\
\hline 35 & $39^{\circ} 44.09^{\prime}$ & $143^{\circ} 47.53^{\prime}$ & 3401 \\
\hline $35 \mathrm{~A}$ & $39^{\circ} 44.10^{\prime}$ & $143^{\circ} 47.59^{\prime}$ & 3401 \\
\hline 36 & $39^{\circ} 55.96^{\prime}$ & $145^{\circ} 33.47^{\prime}$ & 5240 \\
\hline
\end{tabular}

heated for about 30 minutes at $60^{\circ} \mathrm{C}$ to set the Caedax and then sealed with a cover glass. Chert samples were etched in a 5 per cent hydrofluoric acid solution for about five hours and then washed in water with a soft brush to detach radiolarian specimens from the chert. These were boiled in a solution of sodium hexametaphosphate for about 30 minutes, then sieved and mounted on a slide in the same manner as for other samples. One to 10 slides were prepared from each sample to determine the relative abundance of taxa present in a given assemblage.

This relative abundance is ordinarily obtained by investigating 500 specimens, which I have categorized as follows: A, abundant (over 80 specimens); C, common (21-80 specimens); F, few (6-20 specimens); R, rare (fewer than 6 specimens); and + , for one or two specimens present in an assemblage of more than 2000 radiolarian specimens. Additionally, the following symbols are used: X, for the presence of taxa when the total number of individuals is less than 500 in a given sample; - for the absence of taxa where expected; when the dash is used the assemblage consists of more than 2000 individuals; $\bigcirc$ for the presence of reworked specimens from older deposits; and a blank indicates absence of taxa.

The total abundance of specimens ( $>63 \mu$ in each sample) and the state of preservation of radiolarians in each sample are indicated as follows: A, abundant (more than 2000 specimens in one slide); C, common (500-2000); F, few (100-500); R, rare (less than 100); G, good (more than half the specimens are unbroken, and there is no corrosion); $\mathrm{M}$, moderate (more than half the specimens are fragmented or there is some evidence of corrosion); and P, poor (most of the specimens are fragmented or strongly corroded).

The position of those specimens which are needed for further taxonomic scrutiny was recorded on a strewn slide by the use of an England Finder (Riedel and Foreman, 1961).

The slides used for the present investigation are in the collections of the Institute of Geology and Paleontology, Tohoku University, Sendai, Japan.

\section{BIOSTRATIGRAPHIC FRAMEWORK}

The stratigraphic ranges of radiolarians recovered during this leg correspond well with those reported from DSDP Site 310 by Foreman (1975) for the late Miocene to Pleistocene interval. Therefore I am using the zones designated by her in this report. From the middle to late Miocene interval, I use the zones reported by Riedel and Sanfilippo $(1970,1971,1978)$. 
The definitions of these zones are as follow:

Botryostrobus aquilonaris Zone, Hays, 1970

The base is defined by the occurrence of Botryostrobus quilonaris ( = Eucyrtidium tumidulum of Hays, 1970; = Artostrobium miralestense of Kling, 1973; = Artostrobium tumidulum of Foreman, 1975) following the extinction of Axoprunum angelinum (= Stylatractus universus of Hays, 1970). This zone covers the last 0.4 m.y. (Hays and Shackleton, 1976).

\section{Axoprunum angelinum Zone, Hays, 1970}

This zone is defined by the presence of Axoprunum angelinum subsequent to the extinction of Eucyrtidium matuyamai. This zone spans a time interval between 0.9 and 0.4 m.y. (modified from Hays, 1970).

Eucyrtidium matuyamai Zone, Hays, 1970, emend. Foreman, 1973

This zone is defined by the complete evolutionary range of Eucyrtidium matuyamai, which spans from 1.8 m.y. to 0.9 m.y. (modified from Hays, 1970). The base of this zone corresponds to the middle of the Olduvai paleomagnetic event-that is, nearly the Pliocene/ Pleistocene.

Lamprocyrtis heteroporos Zone, Hays, 1970, emend. Foreman, 1975

The base is defined by the occurrence of Lamprocyrtis heteroporos ( = Lamprocyclas heteroporos in Hays, 1970) subsequent to the extinction of Stichocorys peregrina (2.8 m.y., Hays, 1970) and the top by the first morphotypic evolutionary appearance of Eucyrtidium matuyamai.

\section{Sphaeropyle langii Zone, Foreman, 1975}

The base is defined by the first morphotypic appearance of Sphaeropyle langii and the top by the base of the overlying Lamprocyrtis heteroporos Zone.

Stichocorys peregrina Zone, Riedel and Sanfilippo, 1970, emend. Foreman, 1975

The base is defined by the earliest evolutionary appearance of Stichocorys peregrina and the top by the base of the Sphaeropyle langii Zone.

\section{Ommatartus penultimus Zone, Riedel and Sanfilippo, 1970}

The base is defined by the earliest evolutionary appearance of Ommatartus penultimus and the top by the base of the Stichocorys peregrina Zone.

Ommatartus antepenultimus Zone, Riedel and Sanfilippo, 1970, emend. Riedel and Sanfilippo, 1978

The base is defined by the earliest evolutionary appearance of Ommatartus hughesi and the top by the base of the Ommatartus penultimus Zone.
Cannartus petterssoni Zone, Riedel and Sanfilippo, 1970, emend. Riedel and Sanfilippo, 1978

The base is defined by the earliest morphotypic appearance of Cannartus petterssoni and the top by the base of the Ommatartus antepenultimus Zone.

Dorcadospyris alata Zone, Riedel and Sanfilippo, 1970, emend. Riedel and Sanfilippo, 1971

The base is defined by the earliest evolutionary appearance of Dorcadospyris alata and the top by the base of the Cannartus petterssoni Zone.

\section{RECOVERY OF RADIOLARIANS}

\section{Site 434}

Sediments recovered from Site 434 contain few to rare, moderate to poorly preserved radiolarians, except for Samples 434-1, CC and 434-2-1, 15-17 cm, which contain abundant and well-preserved and common and moderately preserved radiolarians, respectively (see Table 2). Reworked species from a middle Miocene or lower Miocene interval were dominant in samples from the lower part of Hole 434B.

\section{Hole 434}

Core 1 contains Lamprocyrtis haysi and Lychnocanoma sp., whose ranges are restricted to an interval from the Botryostrobus aquilonaris to the Axoprunum angelinum Zone. This core lacks Spongodiscus sp., whose uppermost occurrence is in the middle part of the B. aquilonaris Zone. Moreover, the Core 1 core catcher, which contains the most abundant and well-preserved radiolarians at this site, lacks both Stylacontarium acquilonium and $A$. angelinum, although Sections 1 to 3 contain both of them. Therefore in Core 1 both $S$. acquilonium and $A$. angelinum are judged to be reworked and the entire core is assigned to the $B$. aquilonaris Zone. Core 2 is assigned to the Eucyrtidium matuyamai Zone by the occurrence of E. matuyamai. Between Cores 1 and 2 there is a hiatus which encompasses the upper part of the $E$. matuyamai Zone, the $A$. angelinum Zone, and the lower part of the $B$. aquilonaris Zone.

Core 7 contains the uppermost occurrence of Stichocorys peregrina, indicating that the upper boundary of the Sphaeropyle langii Zone should occur between Cores 6 and 7 ( $45-54 \mathrm{~m})$; an interval from Cores 3 to 6 is assigned to the Lamprocyrtis heteroporos Zone. The bottom of this hole is still within the Sphaeropyle langii Zone.

Diatom analyses showed biostratigraphic breaks between Cores 12 and 13,14 and 15,17 and 18, and 28 and 29 (Harper, this volume). Radiolarian analyses revealed no distinct faunal repetitions for these intervals. However, Cores 13,18, and 19 contain more numerous specimens in a better state of preservation than do the cores immediately above them. The occurrence of radiolarian specimens seems to support the scheme suggested from the diatom data. 


\section{Hole 434A}

Only two cores were obtained from this hole. The upper one, Core 1, contains many radiolarian specimens, and intervals from Sections 1-1 to 1-3 and 1-5 to 1, CC are assigned to the Botryostrobus aquilonaris and Lamprocyrtis heteroporos zones, respectively. This core contains a hiatus between Sections 3 and 5 in the same stratigraphic position as that in Hole 434. The missing interval ranges from early to late Pleistocene. In Core 2 radiolarians are rare, and no age assignment was obtained.

\section{Hole 434B}

Radiolarian recovery from this hole was rare, especially in the upper and lower parts (Cores 2 through 9 and 24 through 37 ).

Stichocorys peregrina is the predominant species throughout these cores. The lowermost occurrence of Sphaeropyle langii is in Core 35 and of Stylacontarium acquilonium in Core 37, but both $S$. langii and $S$. acquilonium are absent from Cores 17 through 34 . Moreover, Ommatartus avitus $(15, \mathrm{CC}), O$. penultimus $(15$, $\mathrm{CC}$ and 26-1), and $O$. hughesi (26-1 and 28, CC) in those intervals above Core 35 . Therefore Cores 2 to 16 and 17 to 34 are assigned to the Sphaeropyle langii and Stichocorys peregrina zones, respectively. Cores 35 to 37 are considered to correspond to the Sphaeropyle langii Zone, although some doubt still remains concerning the possibility that $S$. langii and $S$. acquilonium may be mixed downward.

\section{Site $\mathbf{4 3 5}$}

Sediments containing a few to common, wellpreserved radiolarians were recovered from this site (see Table 3). The uppermost occurrence of Axoprunum angelinum is in Section 435-4-1. Eucyrtidium matuyamai is present in Sections 435-6-4 through 435-8, CC, and Stichocorys peregrina occurs below Core 435-13. In Core 435A-11, the lowermost core from this site, Lamprocyrtis heteroporos and Stylacontarium acquilonium are present, although Sphaeropyle langii is absent. Both $L$. heteroporos and Stylacontarium acquilonium first appear later than Sphaeropyle langii. Therefore Cores 435-1 and 435-2 are assigned to the Botryostrobus aquilonaris Zone; Core 435-4 through Section 435-6-2 to the $A$. angelinum Zone; Section 435-6-4 through the core catcher of Core 8 to the E. matuyamai Zone; Cores 435-9 through 435-12 to the L. heteroporos Zone; and Cores 435-13 through 435-16 as well as 435A-2 through $435 \mathrm{~A}-11$ to the $S$. langii Zone.

\section{Site 436}

Common to abundant, well-preserved radiolarians were recovered from Core 1 through Section 1 of Core 39 (see Tables 4 and 5 and Figure 1). Radiolarians are absent from Core 39, Section 3 through the upper part of Core 41 . In the lower part of Cores 41 and 42 they are few to rare and are very poorly preserved.

In an interval from Core 1 through the upper part of Core 4 (from 0 to approximately $35 \mathrm{~m}$ ), there is an as- semblage correlative to the Botryostrobus aquilonaris Zone (0-0.4 m.y.). The lower part of Core 4 through the middle part of Core 7 (from approximately 35 to $65 \mathrm{~m}$ ) is assigned to the Axoprunum angelinum Zone (0.4-0.9 m.y.), and the lower part of Core 7 through the middle part of Core 11 (from approximately 65 to $100 \mathrm{~m}$ ) to the Eucyrtidium matuyamai Zone (0.9-1.8 m.y.). The lower part of Core 11 through Core 17 (from approximately 100 to $160 \mathrm{~m}$ ) and Cores 18 through 28 (from approximately 160 to $260 \mathrm{~m}$ ) are assigned to the Lamprocyrtis heteroporos and Sphaeropyle langii zones, respectively. Cores 29 and 30 (from approximately 260 to $280 \mathrm{~m}$ ), Core 31 (from approximately 280 to $290 \mathrm{~m}$ ), and Core 32 through the upper part of Core 33 (from approximately 290 to $305 \mathrm{~m}$ ) correspond to the Stichocorys peregrina, Ommatartus penultimus, and $O$. antepenultimus zones, respectively. The Cannartus petterssoni Zone occurs in the interval from the middle part of Core 33 through the upper part of Core 34 (from approximately 305 to $316 \mathrm{~m}$ ), and the lower part of Core 34 through Section 1 of Core 39 (from 316 to $360 \mathrm{~m}$ ) is assigned to the Dorcadospyris alata Zone. Section 2 of Core 39 contains a few fragments of orosphaerid radiolarians, but there is no age indicator. In the lower part of Core 41 there are abundant but very poorly preserved Cretaceous radiolarians in a few fragments of chert and pale olive clay surrounding chert. This assemblage, which includes only Cretaceous forms (Table 5) and lacks Cenozoic forms, suggests that the age of the lower part of Cores 41 and 42 is late Albian or Cenomanian (Dictiomitra somphedia Zone, Foreman, 1975).

\section{EVOLUTIONAL LINEAGES}

The suggested evolutionary transitions of taxa reported here are important for future biostratigraphic application. Some of them are newly observed, whereas others have been confirmed by workers in other areas. They are as follows:

1) A lineage leading from Cannartus mammifer through $C$. laticonus, Ommatartus antepenultimus, $O$. penultimus, and $O$. avitus to $O$. tetrathalamus. This lineage is a part of the $C$. prismaticus-O. tetrathalamus lineage which was described previously (Riedel, 1959; Riedel and Sanfilippo, 1970). In the materials cored during this leg, the transition from $C$. laticonus to $O$. antepenultimus was not detected. The transition from the ancestral $C$. violina to $C$. mammifer may be found in horizons still lower than the lowest Neogene horizon examined here.

2) A lineage leading from $C$. sp. A through $C$. sp. B and $C$. petterssoni to $O$. hughesi. This lineage may begin with C. mammifer.

3) A lineage leading from $C$. sp. C through $O . \mathrm{sp} . \mathrm{C}$ to $O$. didymus. This lineage is apparently a side branch from the $C$. mammifer-O. tetrathalamus lineage mentioned above. The ancestor of this lineage is $C$. laticonus.

4) A lineage leading from $C$. sp. D to $O$. sp. D. Neither ancestors nor descendants of this lineage are known so far. 
TABLE 2

Radiolarians from Site 434

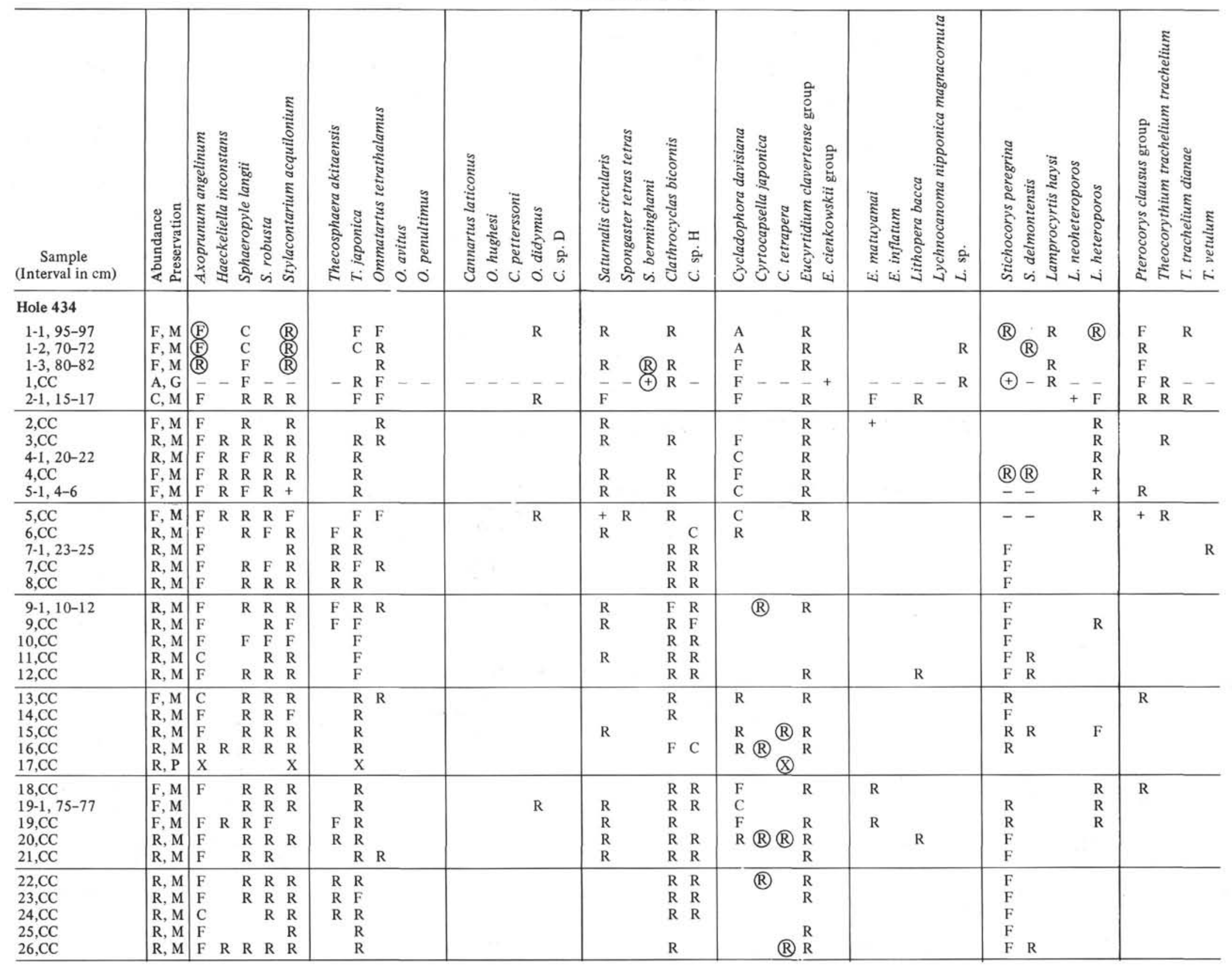




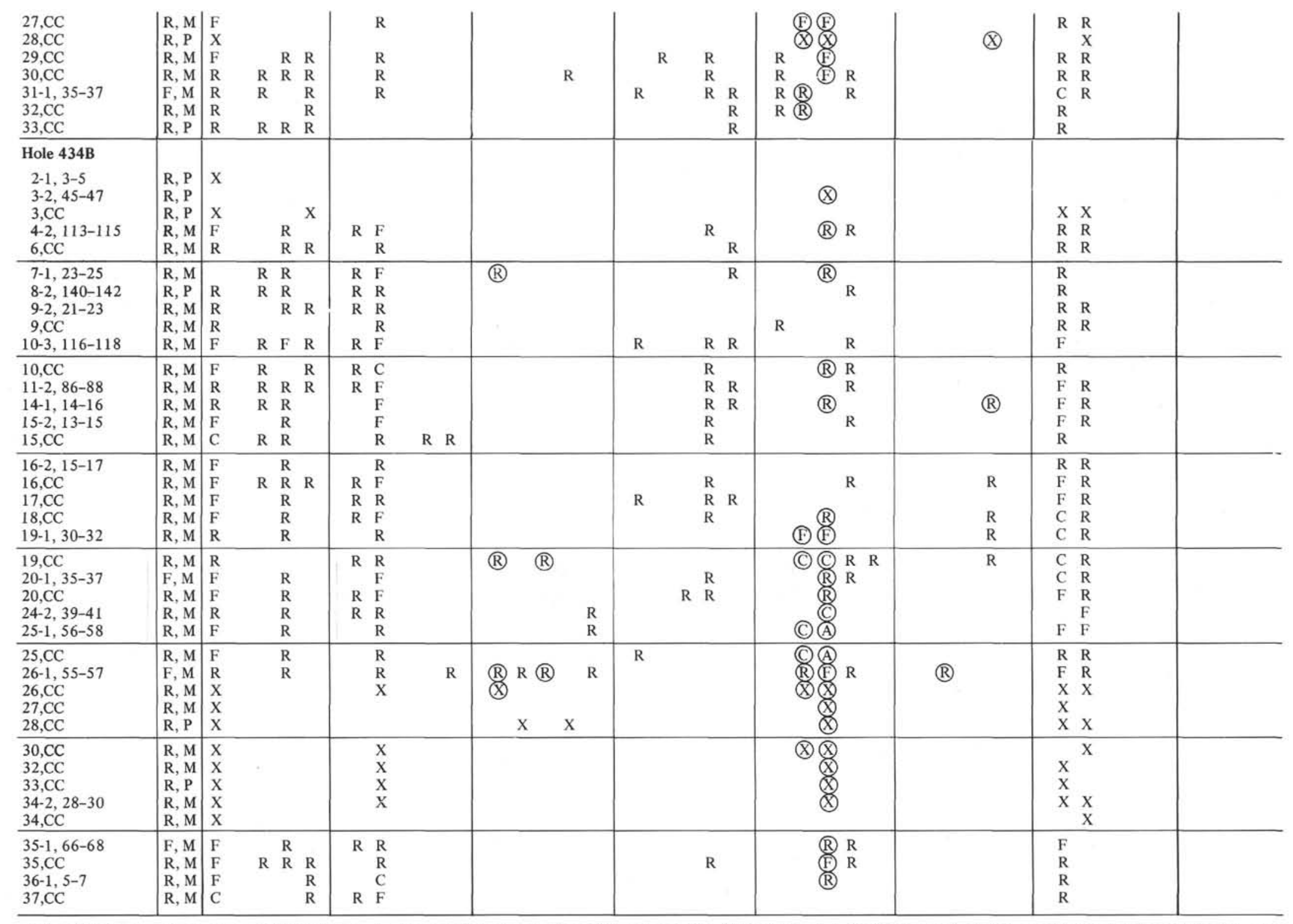

Note: Relative abundance among 500 specimens: A, abundant (over 80 specimens); C, common ( $21-80$ specimens); F, few (6-20 specimens); $R$, rare (fewer than 6 specimens); +1 or 2 specimens in an assemblage of more than 2000 . X, presence of taxa when total number of individuals is less than $500 ;-$, absence of taxa where expected and when assemblage consists of more than 2000 individuals; 1 , presence of reworked specimens from older deposits; a blank, absence of taxa.

Abundance of specimens $(>63 \mu)$ in each sample and preservation: A, abundant (more than 2000); C, common (500-2000); F, few (100-500); R, rare (less than 100), G, good (more than half the specimens unbroken, no corrosion); M, moderate (more than half the specimens are fragmented or there is some corrosion); P, poor (most specimens are fragmented or strongly corroded). 
TABLE 3

Radiolarians from Site $\mathbf{4 3 5}$

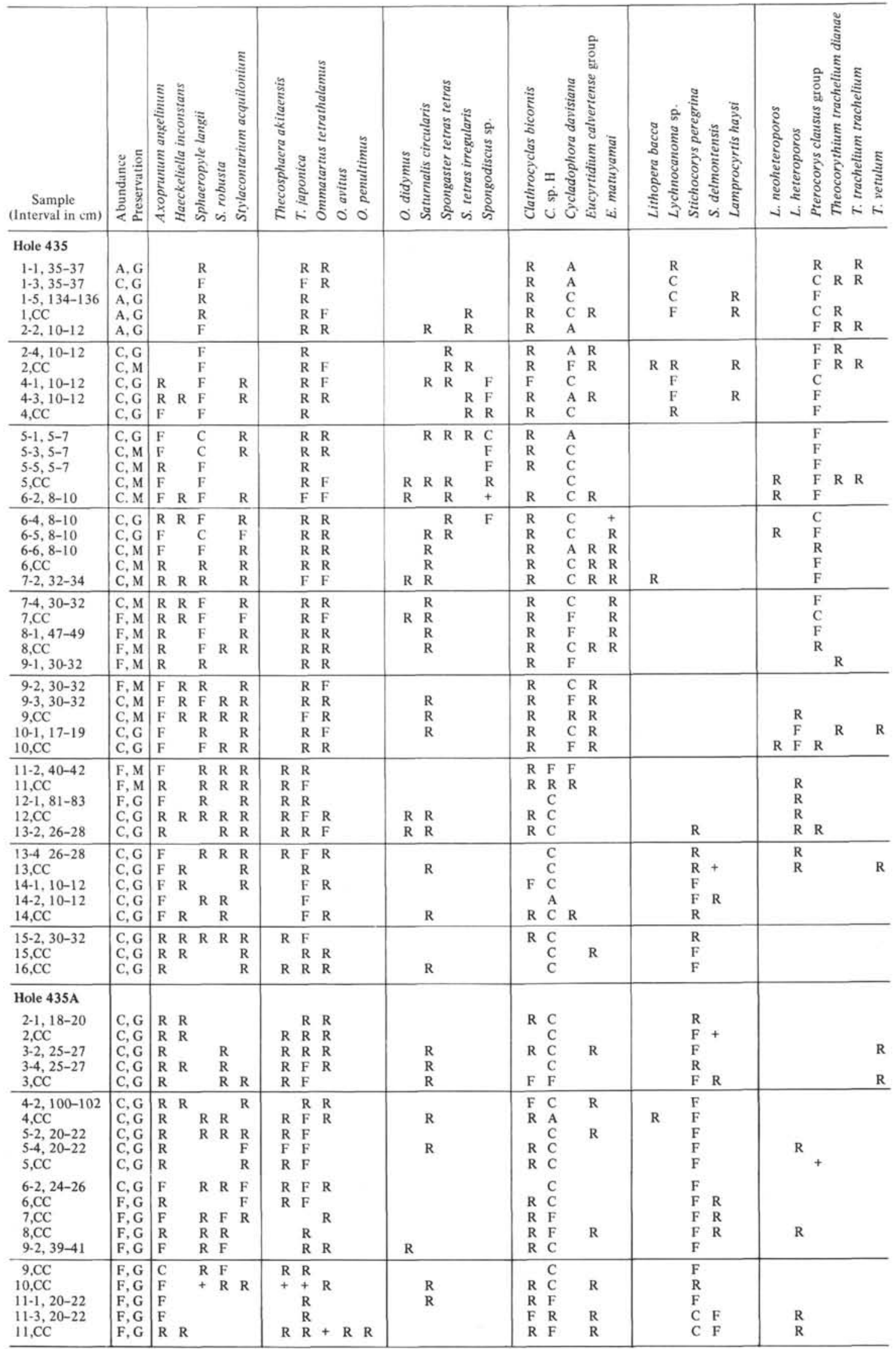

Note: See Note to Table 2 for explanation of symbols. 
5) A lineage beginning with a form which is here included in the Eucyrtidium cienkowskii group to $E$. asanoi $\mathrm{n}$. $\mathrm{sp}$. This series apparently became extinct with the later species.

6) A lineage beginning with $E$. calvertense s. str. to $E$. matuyamai. This relation was reported previously (Hays, 1970; Kling, 1973; Foreman, 1975).

7) A lineage leading from Lithopera renzae through $L$. neotera to $L$. bacca. This lineage is well defined in cores from Site 436. Stichocorys diploconus, which was previously considered to be the ancestor of this lineage (Sanfilippo and Riedel, 1970), was not found in the materials studied here.

8) A lineage leading from $S$. delmontensis to $S$. peregrina. This lineage was reported previously (Riedel and Sanfilippo, 1970).

The transitions from Sphaeropyle robusta to $S$. langii, from $E$. calvertense group to $E$. inflatum, from Lamprocyrtis heteroporos to $L$. neoheteroporos, and from $L$. neoheteroporos to $L$. haysi, which were previously distinguished in other areas, were not confirmed in the present study because of their rare and/or sporadic occurrence. The lineage leading from Spongaster berminghami through $S$. pentas to $S$. tetras was not confirmed either because of the absence of $S$. pentas. Although both Dorcadospyris dentata and D. alata occur with probable transitional forms in the lowermost sample containing Cenozoic radiolarians, an evolutionary transition from the former to the latter is considered to have taken place somewhat lower than the lowest sample cored here.

\section{RADIOLARIAN EVENTS AND POTENTIAL DATUM LEVELS}

Table 6 summarizes the sequence of events observed at Sites 434, 435, and 436, following the sequence established at Site 436, and includes only those events which appear to have a potential stratigraphic significance. In addition to the previously reported datum levels, I note those events that may be of stratigraphical significance, though possibly only local (see also Figure 2).

1) The bottom of Eucyrtidium asanoi. This species evolved from its ancestor in the basal part of the Dorcadopyris alata Zone. The base of this zone is taken to mark the early/middle Miocene boundary. In Japan this species occurs in the lower part of the middle Miocene, whereas no $D$. dentata or $D$. alata have been found in Japanese lower and middle Miocene sediments (unpublished data). Therefore the initial appearance of $E$. asanoi is likely to become useful for the establishment of the early/middle Miocene boundary in Japan and its neighboring areas.

2) The top of Eucyrtidium sp. B. This species disappears in the middle part of the $D$. alata Zone-that is, just below the level of the transition from Lithopera renzae to $L$. neotera.

3) The transition from Cannartus sp. A to Cannartus sp. B. This level occurs at nearly the same horizon as the top of Eucyrtidium sp. B.
4) The bottom of Lychnocanoma nipponica magnacornuta n. subsp. This subspecies first appears in the upper part of the $D$. alata Zone in the materials examined here and in the lower part of the $L$. nipponica Zone ( = Lychnocanium nipponicum in Nakaseko and Sugano, 1973) in Japan (unpublished data).

5) The transition from Cannartus sp. C to Ommatartus sp. C. This level occurs in the uppermost part of the $D$. alata Zone-that is, just below the morphotypic bottom of $C$. petterssoni.

6) The transition from Cannartus sp. B to C. petterssoni. This level is observed in the lower part of the C. petterssoni Zone and below the transition horizon from Lithopera renzae to $L$. bacca.

7) The top of Eucyrtidium yatsuoense. This species disappears in the middle part of the $C$. petterssoni Zone-that is, somewhat above the transition level of Lithopera bacca from L. renzae.

8) The transition from $O$. sp. C to $O$. didymus. This transition occurs slightly below the transition horizon of $C$. petterssoni from $O$. hughesi.

9) The top of Lychnocanoma nipponica magnacornuta. The uppermost occurrence of this subspecies is found in the $O$. antepenultimus Zone.

10) The bottom of Thecosphaera japonica. This level is found at nearly the same horizon as the base of the Stichocorys peregrina Zone. The latter occurs commonly from upper Neogene sequences exposed along the coast of the Sea of Japan and is used as a marker species of the T. japonica Zone (Nakaseko and Sugano, 1973).

11) The top of Cannartus sp. E. The uppermost occurrence of this species is found just above the base of the $S$. peregrina Zone. This relation agrees with that reported from the central North Pacific by Foreman (1975). In her article Cannartus sp. E was reported by the name of Ommatartus sp. cf. C. bassani.

12) The transition from Cannartus sp. D to Ommatartus sp. D. This transition is observed at the horizon at which Sphaeropyle langii occurs initially.

13) The top of L. nipponica nipponica. This horizon is in the interval between the bottoms of $S$. langii and Lamprocyrtis heteroporos.

14) The top of Ommatartus sp. D. This level occurs just above the bottom of $L$. heteroporos in the materials examined here, whereas the top of this species (represented as Ommatartus sp.) was reported to lie below the bottom of L. heteroporos at DSDP Site 310 in the central North Pacific (Foreman, 1975). Because the interval between the top and bottom of both species is very small in Holes 310 and 436, the difference in their stratigraphic ranges is negligible.

15) The top of Thecosphaera akitaensis. This level occurs in the $L$. heteroporos Zone and may be somewhat lower than the top of Clathrocyclas sp. $\mathrm{H}$, as noted below.

16) The top of Clathrocyclas sp. H. This species disappears within the $L$. heteroporos Zone.

17) The bottom of Spongodiscus sp. This species occurs initially nearly the same level at which $E$. matuyamai becomes extinct. This relation agrees well with that reported from DSDP Leg 19 (Ling, 1973). 
TABLE 4

Radiolarians from Site 436 (Neogene)

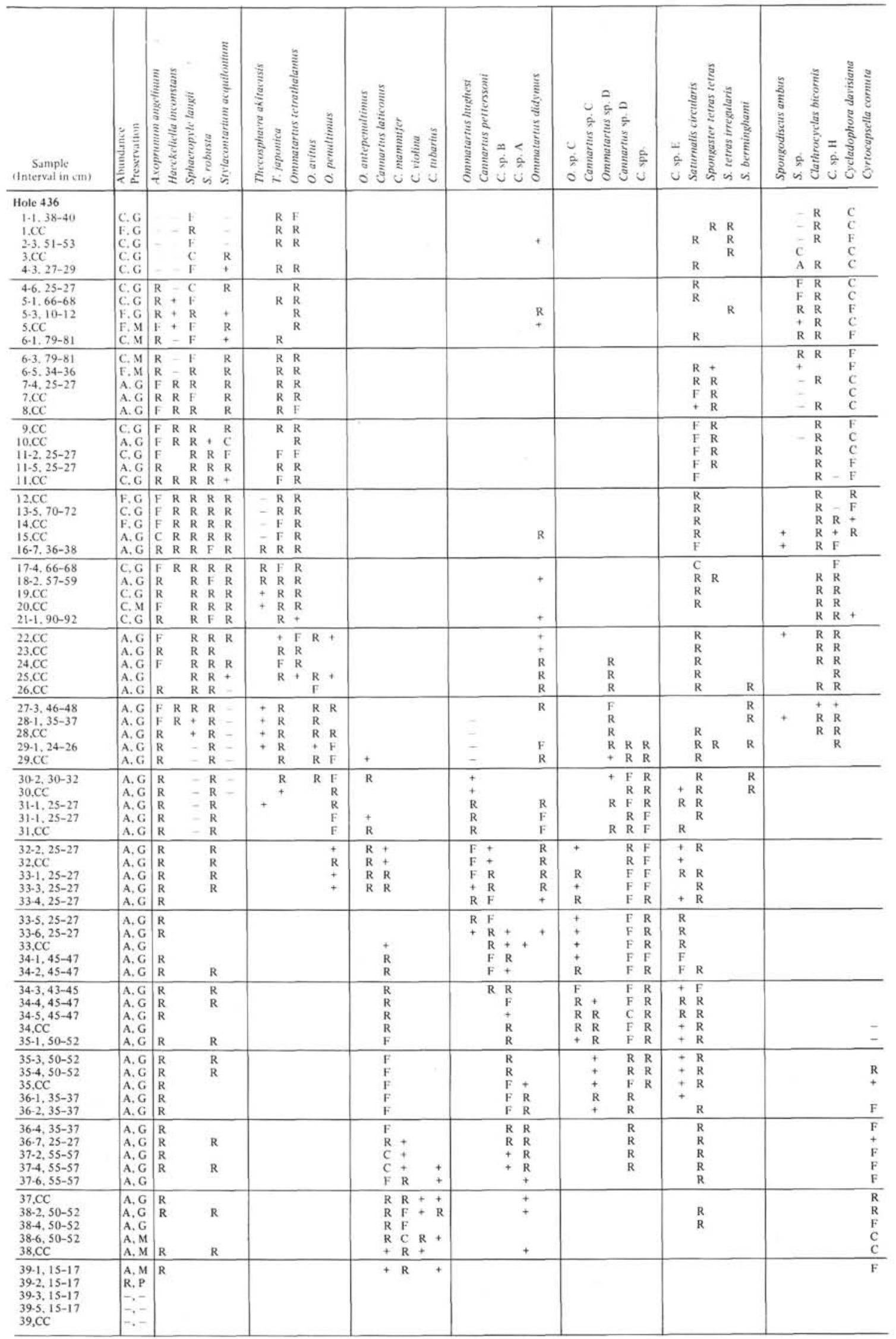


TABLE 4 - Continued

\begin{tabular}{|c|c|c|c|c|c|c|c|c|c|c|c|c|c|c|c|c|c|}
\hline 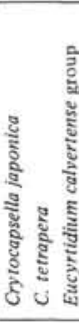 & 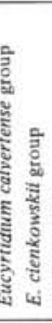 & & 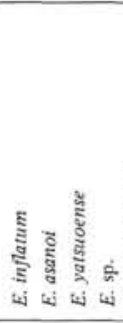 & 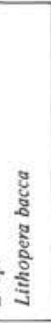 & 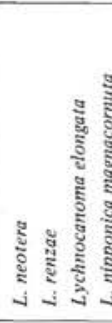 & 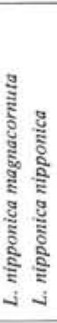 & 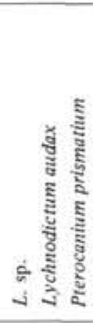 & & & 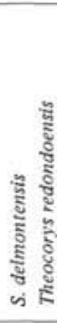 & 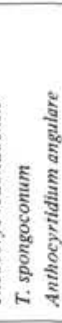 & & 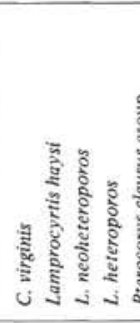 & 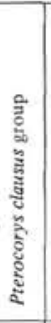 & 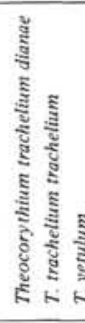 & 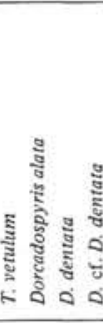 & 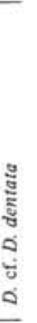 \\
\hline & $\begin{array}{l}\text { R } \\
\text { R }\end{array}$ & & & $\begin{array}{l}\mathrm{R} \\
\mathrm{R} \\
+\end{array}$ & & & $\begin{array}{l}\mathrm{R} \\
\mathrm{F} \\
\mathrm{F} \\
\mathrm{R} \\
\mathrm{F} \\
\end{array}$ & & & & & & $\begin{array}{l}\mathrm{R} \\
\mathrm{R} \\
\mathrm{R}\end{array}$ & $\begin{array}{l}\mathrm{F} \\
\mathrm{R} \\
\mathrm{F} \\
\mathrm{R} \\
\mathrm{F} \\
\end{array}$ & $\begin{array}{r}\mathrm{R} \\
+\quad \mathrm{R} \\
\mathrm{R}+ \\
\mathrm{R} \\
\end{array}$ & & \\
\hline & $\begin{array}{l}R \\
R \\
R \\
R\end{array}$ & & & $\mathrm{R}$ & & & $\begin{array}{l}\mathrm{R} \\
\mathrm{F} \\
\mathrm{R} \\
\mathrm{R} \\
\mathrm{R}\end{array}$ & & & & & & $\begin{array}{lll}R & R & \\
R & & \\
R & & \\
R & R & \\
& & \\
\end{array}$ & $\begin{array}{l}\mathrm{C} \\
\mathrm{C} \\
\mathrm{F} \\
\mathrm{F} \\
\mathrm{C} \\
\end{array}$ & + & & \\
\hline & $\begin{array}{ll}R & R \\
R & R \\
R\end{array}$ & & & & & & $\begin{array}{l}y \\
\bar{y}\end{array}$ & & & & $\begin{array}{l}\mathrm{R} \\
\mathrm{R}\end{array}$ & & $\begin{array}{rl} & \\
& \\
& \mathrm{R} \\
+ & + \\
\mathrm{R} & \mathrm{R} \\
\end{array}$ & $\begin{array}{l}\mathrm{C} \\
\mathrm{R} \\
\mathrm{F} \\
\mathrm{A} \\
\mathrm{C}\end{array}$ & $\begin{array}{c}\mathrm{R} \\
\mathrm{R} \\
\mathrm{T} \\
\mathrm{R}\end{array}$ & & \\
\hline $\begin{array}{l}\mathrm{R} \\
+ \\
\mathrm{R} \\
\mathrm{R}\end{array}$ & 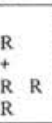 & & & R & & & $\begin{array}{l}R \\
R\end{array}$ & & & & & & $\begin{array}{r}\mathrm{R} \\
+\mathrm{F} \\
\mathrm{R} \\
+\mathrm{R} \\
\mathrm{R}\end{array}$ & $\begin{array}{l} \\
R \\
R \\
R\end{array}$ & $\begin{array}{ll} & R \\
R & R \\
& R \\
& R \\
& R \\
\end{array}$ & $\begin{array}{l}\mathbf{R} \\
\mathrm{R} \\
\mathrm{R} \\
\mathrm{R} \\
\mathrm{R} \\
\mathrm{R}\end{array}$ & \\
\hline & $\begin{array}{lll}R & \\
R & R \\
R & \end{array}$ & & & $\begin{array}{l}R \\
+ \\
R\end{array}$ & & & $\begin{array}{l}\mathrm{R} \\
+\end{array}$ & & & & & & $\begin{array}{l}R \\
R \\
R \\
F \\
R\end{array}$ & & $\begin{array}{l}R \\
R \\
R \\
R \\
R \\
F\end{array}$ & $\begin{array}{l}R \\
R \\
R \\
R \\
R \\
F\end{array}$ & \\
\hline & $\begin{array}{l}\mathrm{R} \\
\mathrm{R} \\
\mathrm{R} \\
\mathrm{R} \\
\mathrm{R} \\
\mathrm{R}\end{array}$ & & & $\begin{array}{l}\mathrm{R} \\
\mathrm{R} \\
+ \\
\end{array}$ & & & R & & $\begin{array}{l}\mathrm{F} \\
\mathrm{F} \\
\mathrm{C} \\
\mathrm{A}\end{array}$ & $\mathrm{R}$ & & & $\begin{array}{l}\mathrm{R} \\
\mathrm{R} \\
\mathrm{R} \\
\mathrm{R} \\
\mathrm{F}\end{array}$ & & & $\begin{array}{l}R \\
R \\
\text { F } \\
F \\
\text { F } \\
R\end{array}$ & \\
\hline & $\begin{array}{l}\mathrm{R} \\
\mathrm{R}\end{array}$ & & & $\begin{array}{l}\mathrm{R} \\
\mathrm{R}\end{array}$ & & & $\begin{array}{l}+ \\
+ \\
+\end{array}$ & & $\begin{array}{l}\mathrm{A} \\
\mathrm{A} \\
\mathrm{A} \\
\mathrm{A} \\
\mathrm{A}\end{array}$ & $\begin{array}{l}\mathrm{R} \\
\mathrm{R} \\
\mathrm{R} \\
\mathrm{R} \\
\mathrm{R}\end{array}+$ & & & $\begin{array}{l}\mathrm{F} \\
\mathrm{R} \\
\mathrm{R}\end{array}$ & & & $\begin{array}{l}\mathrm{F} \\
\mathrm{R} \\
\mathrm{R} \\
+ \\
+\end{array}$ & \\
\hline & $\begin{array}{ll}R & R \\
R & R \\
F & R \\
R & R \\
R & \\
\end{array}$ & & & $\begin{array}{l}\mathrm{R} \\
\mathrm{R} \\
\mathrm{R} \\
\mathrm{R} \\
\mathrm{R} \\
\end{array}$ & & $\begin{array}{l}\mathrm{R} \\
\mathrm{R} \\
\mathrm{R} \\
\mathrm{R}\end{array}$ & + & & $\begin{array}{l}\mathrm{C} \\
\mathrm{A} \\
\mathrm{A} \\
\mathrm{C} \\
\mathrm{C} \\
\mathrm{C}\end{array}$ & $\begin{array}{l}\mathrm{R} \\
\mathrm{F} \\
\mathrm{F} \\
\mathrm{F} \\
\mathrm{F}\end{array}$ & & & & & & $\begin{array}{l}\mathrm{R} \\
+ \\
+ \\
+\end{array}$ & \\
\hline & $\begin{array}{lll}+ & \\
+ & \\
& + \\
R & R \\
R & R \\
F & R \\
\end{array}$ & & & $\begin{array}{l}\mathrm{R} \\
\mathrm{F} \\
\mathrm{F} \\
\mathrm{R} \\
\mathrm{R} \\
\mathrm{R}\end{array}$ & & $\begin{array}{l}-R \\
=R \\
=R \\
=+ \\
=\end{array}$ & $\begin{array}{l}\mathrm{R} \\
+ \\
\mathrm{R}\end{array}$ & & $\begin{array}{l}\mathrm{C} \\
\mathrm{C} \\
\mathrm{F} \\
\mathrm{R} \\
\mathrm{R} \\
\end{array}$ & $\begin{array}{ll}\mathrm{F} & + \\
\mathrm{C} & \\
\mathrm{C} & \\
\mathrm{C} & \mathrm{R} \\
\mathrm{F} & \mathrm{R}\end{array}$ & & & & & & $\begin{array}{l}+ \\
+ \\
\mathrm{R} \\
\mathrm{R}\end{array}$ & \\
\hline $\begin{array}{r}R \\
R \\
R \\
-R \\
-\quad R \\
-\quad\end{array}$ & $\begin{array}{ll}R & R \\
R & R \\
R & R \\
R & R \\
R & R \\
R & F \\
\end{array}$ & & & $\begin{array}{l}R \\
R \\
R\end{array}$ & - & $\begin{array}{ll}\mathrm{R} & \mathrm{R} \\
+ & \mathrm{R} \\
\mathrm{R} & + \\
\mathrm{R} & \mathrm{R} \\
\mathrm{F} & \\
\end{array}$ & $\begin{array}{l}\mathrm{R} \\
+ \\
+ \\
\mathrm{R} \\
\mathrm{R} \\
\end{array}$ & & $\begin{array}{l}\mathrm{R} \\
\mathrm{R} \\
\mathrm{R} \\
\mathrm{R} \\
\mathrm{R} \\
\end{array}$ & $\begin{array}{ll}\mathrm{F} & + \\
\mathrm{F} & + \\
\mathrm{F} & \\
\mathrm{F} & \mathrm{R} \\
\mathrm{F} & \mathrm{R}\end{array}$ & & & & & & $\begin{array}{l}R \\
+ \\
+\end{array}$ & \\
\hline $\begin{array}{l}++R \\
C=R \\
+C=R \\
C=R \\
R=R \\
\end{array}$ & $\begin{array}{ll}\mathrm{R} & \mathrm{R} \\
\mathrm{R} & \mathrm{R} \\
\mathrm{R} & \mathrm{R} \\
\mathrm{R} & \mathrm{R} \\
\mathrm{R} & \mathrm{R} \\
\end{array}$ & & $\begin{array}{l}\mathrm{R} \\
\mathrm{R} \\
\mathrm{R} \\
+ \\
\end{array}$ & $\begin{array}{l}\mathrm{R} \\
\mathrm{F} \\
+ \\
\mathrm{R} \\
\end{array}$ & $\begin{array}{l}- \\
\bar{F} \\
\text { R } \\
\mathrm{F}\end{array}$ & $\begin{array}{ll}R & R \\
R & R \\
R & R \\
R & R \\
R & R \\
\end{array}$ & $\begin{array}{l}\mathrm{R} \\
\mathrm{R} \\
\mathrm{R} \\
\mathrm{F}\end{array}$ & & $\begin{array}{l}\mathrm{R} \\
\mathrm{R} \\
\mathrm{R}\end{array}$ & $\begin{array}{l}\mathrm{F}+ \\
\mathrm{F} \\
\mathrm{F} \\
\mathrm{F} \\
\mathrm{F}\end{array}$ & & & & & & & \\
\hline $\begin{array}{l}F+R \\
C+R \\
F \\
F-R \\
F-R \\
F-B\end{array}$ & $\begin{array}{lll}R & R \\
F & R \\
R & R \\
R & R \\
R & R \\
R & F \\
\end{array}$ & & $\begin{array}{r}R \\
R \\
R \\
R \\
-R \\
-R \\
\end{array}$ & $\begin{array}{l}R \\
R \\
R \\
R \\
F \\
F\end{array}$ & $\begin{array}{l}\mathrm{R} \\
\mathrm{R} \\
\mathrm{R} \\
\mathrm{F} \\
\mathrm{C}- \\
\end{array}$ & $\begin{array}{ll}R & F \\
R & R \\
R & R \\
& F \\
R & F \\
\end{array}$ & $\begin{array}{l}\mathrm{R} \\
\mathrm{R} \\
\mathrm{F} \\
\mathrm{R} \\
\mathrm{R}\end{array}$ & & $\begin{array}{l}\mathrm{R} \\
\mathrm{R} \\
\mathrm{R} \\
\mathrm{R} \\
\mathrm{R}\end{array}$ & $\begin{array}{l}\mathrm{F} \\
\mathrm{C} \\
\mathrm{C} \\
\mathrm{C} \\
\mathrm{F}+\end{array}$ & & & & & & + & \\
\hline $\begin{array}{ll}\mathrm{C} & - \\
\mathrm{C} & - \\
\mathrm{C} & + \\
\mathrm{F} & \mathrm{C} \\
\mathrm{F} & + \\
\mathrm{F} & \mathrm{F}\end{array}$ & $\begin{array}{r}\mathrm{R} \\
\mathrm{R} \\
+\quad \mathrm{R} \\
+\quad \mathrm{R} \\
\mathrm{F} \\
\end{array}$ & & $\begin{array}{l}-R \\
-R \\
R+R \\
++R \\
+\end{array}$ & $\begin{array}{l}\text { R } \\
R \\
+ \\
+\end{array}$ & $\begin{array}{lll}F & \bar{r} \\
F & R \\
F & R \\
F & R \\
R & R \\
\end{array}$ & $\begin{array}{ll}\mathrm{R} & \mathrm{F} \\
\mathrm{R} & \mathrm{F} \\
& \mathrm{R} \\
& \mathrm{R} \\
& \mathrm{R} \\
\end{array}$ & $\begin{array}{l}\mathrm{R} \\
\mathrm{R} \\
\mathrm{R} \\
\mathrm{R} \\
\mathrm{R} \\
\end{array}$ & $\begin{array}{l}\text { - } \\
-\end{array}$ & & $\begin{array}{l}\mathrm{F} \\
\mathrm{F}+ \\
\mathrm{F}+ \\
\mathrm{F} \\
\mathrm{R}+\end{array}$ & & & & & & + & \\
\hline 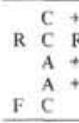 & $\begin{array}{ll}+ & \mathrm{F} \\
\mathrm{R} & \mathrm{F} \\
+ & \mathrm{F} \\
+ & \mathrm{F} \\
\end{array}$ & & $\begin{array}{lllll}\mathrm{R} & \mathrm{F} & \mathrm{R} & \\
\mathrm{R} & \mathrm{R} & & \\
\mathrm{R} & & & \\
\mathrm{R} & & & & + \\
& & \mathrm{F} & \mathrm{R} \\
& \mathrm{R} & \mathrm{R} & \mathrm{F} \\
\end{array}$ & & $\begin{array}{|lll|}\mathrm{R} & \mathrm{C} & \\
\mathrm{R} & \mathrm{F} & \\
\mathrm{R} & \mathrm{F} & \\
& \mathrm{R} & \mathrm{F} \\
& \mathrm{R} & \mathrm{F} \\
\end{array}$ & $\begin{array}{l}\mathrm{F} \\
+\end{array}$ & $\begin{array}{l}\mathrm{R} \\
\mathrm{R} \\
\mathrm{R} \\
\mathrm{R} \\
\mathrm{R}\end{array}$ & $\begin{array}{l}-\bar{c} \\
\bar{F} \\
\mathrm{R}\end{array}$ & & $\begin{array}{l}R \\
R \\
R \\
R \\
R \\
F\end{array}$ & & & $\begin{array}{l}\mathrm{R} \\
\mathrm{R}\end{array}$ & & & $\dot{R}$ & \\
\hline 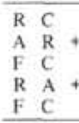 & 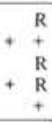 & & $\begin{array}{lll}R & R & F \\
R & F & F \\
& F & R \\
& R & R \\
& R & F \\
& R & F \\
\end{array}$ & 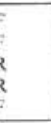 & $\begin{array}{ll}R & F \\
& C \\
R & R \\
R & F \\
R & F \\
\end{array}$ & 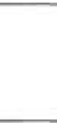 & $\begin{array}{l}\mathrm{R} \\
\mathrm{F} \\
\mathrm{F} \\
\mathrm{F} \\
\mathrm{R}\end{array}$ & $\begin{array}{l}\mathrm{R} \\
\mathrm{R} \\
\mathrm{R}\end{array}$ & & $\begin{array}{l}\mathrm{C} \\
\mathrm{F} \\
\mathrm{F} \\
\mathrm{R} \\
\mathrm{R}\end{array}$ & $\begin{array}{l}\mathrm{R} \\
\mathrm{R} \\
\end{array}$ & $\begin{array}{l}\mathrm{R} \\
\mathrm{R} \\
\mathrm{F} \\
+ \\
\end{array}$ & $\begin{array}{l}R \\
F \\
R \\
R \\
R\end{array}$ & & & $\begin{array}{l}\mathrm{R} \\
\mathrm{R} \\
\mathrm{R} \\
\mathrm{R} \\
\end{array}$ & $\begin{array}{l}\mathrm{R} \\
\mathrm{F} \\
\mathrm{F} \\
\mathrm{F} \\
\mathrm{R} \\
\end{array}$ \\
\hline C C & & & $F$ & 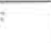 & R F & & $\bar{R}$ & & & $\mathrm{R}$ & + & $\mathrm{R}$ & $R$ & & & $R+C$ & \\
\hline
\end{tabular}


TABLE 5

Radiolarians from Site 436 (Cretaceous)

\begin{tabular}{|c|c|c|c|c|}
\hline Core-Section & \multicolumn{3}{|c|}{$41-1$} & $42-1$ \\
\hline Sample Interval $(\mathrm{cm})$ & $31-32$ & $34-35$ & $47-48$ & $16-17$ \\
\hline Lithology & Clay & Clay & Chert & Chert \\
\hline Artostrobium cf. uruna & $\mathrm{X}$ & $\mathrm{x}$ & & \\
\hline Cryptamphorella conara & $\mathrm{x}$ & $\mathrm{x}$ & $\mathrm{x}$ & \\
\hline Dictyomitra pseudomacrocephala & $\mathrm{X}$ & $\mathrm{x}$ & & \\
\hline D. sp. cf. D. tekschaensis & & $\mathrm{x}$ & & \\
\hline D. spp. & $\mathrm{x}$ & $\mathrm{x}$ & $\mathrm{x}$ & $\mathrm{x}$ \\
\hline Hemicryptocapsa prepolyhedra & $\mathrm{x}$ & & & \\
\hline Holocryptocanium barbui & $\mathrm{x}$ & & & \\
\hline Lithocampe elegantissima & & $\mathrm{x}$ & & \\
\hline Lithomelissa (?) petila & $\mathrm{x}$ & & & \\
\hline Protunuma (?) sp. & $\mathrm{x}$ & & & \\
\hline Spongosaturnalis hueyi group & $\mathrm{x}$ & $\mathrm{x}$ & & \\
\hline$S$. spp. & $\mathrm{x}$ & $\mathrm{X}$ & $\mathrm{x}$ & \\
\hline
\end{tabular}

Note: $\mathrm{X}=$ presence of taxa when the total number of individuals is less than 500 in a given sample.

18) The bottom of Lychnocanoma sp. The lowermost occurrence of this species is in the Axoprunum angelinum Zone.

19) The top of Spongodiscus sp. Although the top level of this species and that of Stylacontarium acquilonium occur at approximately the same horizon in the materials reported here, the former was found to be younger than the latter in sediments cored by DSDP Leg 19 in the North Pacific and the Bering Sea (Ling, 1973). Because my sampling intervals are much wider than those of Ling, the top of Spongodiscus sp. could also be higher than the top of $S$. acquilonium.

\section{CORRELATION WITH PREVIOUSLY ESTABLISHED ZONES IN JAPAN}

Four radiolarian zones have been established for Neogene formations in Japan, based on the concurrence and dominance of characteristic species (Nakaseko and Sugano, 1973). They are, in upward sequence, the $\mathrm{Me}$ littosphaera magnaporulosa, Cyrtocapsella tetrapera, Lychnocanium nipponicum - = Lychnocanoma nipponica (s. 1.) in this report-and Thecosphaera japonica zones.

The base of the $T$. japonica Zone is defined by the first occurrence of $T$. japonica, which occurs at the base of the Stichocorys peregrina Zone in the materials of this leg. Because the lower three zones of Nakaseko and Sugano are in fact defined as being assemblage zones, the lower and upper limits of the C. tetrapera Zone could not be distinguished clearly by means of a single taxon. But this zone is approximately correlated with the lower and middle parts of the Dorcadospyris alata Zone, based on the following facts: (1) Lithopera ren$z a e$, which was described as restricted to the $C$. tetrapera Zone (Nakaseko and Sugano, 1973), was found in the lower and middle parts of the D. alata Zone. (2) The top of Eucyrtidium inflatum, which had been reported to lie above the uppermost common occurrence of C. tetrapera, which in turn defines the top of the $C$. tetrapera Zone (Oda and Sakai, 1977), was found in the same level as the top of C. tetrapera.
The relationship between the zonation used in this report and that previously established in Japan is shown in Figure 2.

\section{TAXONOMY (CENOZOIC FORMS)}

All the type specimens are deposited in the Institute of Geology and Paleontology, Tohoku University, Sendai, Japan. The initials preceding catalogue numbers (IGPS) are those of the Institute of Geology and Paleontology, Sendai.

Subclass RADIOLARIA Müller, 1858

Superorder POLYCYSTINA Ehrenberg, 1838, emend. Riedel, 1967

Order SPUMELLARIA Ehrenberg, 1875

Family ACTINOMMIDAE Haeckel, 1862, emend. Riedel, 1967

Subfamily ACTINOMMINAE Haeckel, 1862, emend. Petrushevskaya and Kozlova, 1972

Genus AXOPRUNUM Haeckel, 1887

Axoprunum angelinum (Campbell and Clark)

(Plate 2, Figures 1a, 1b)

Stylosphaera angelina Campbell and Clark, 1944, p. 12, pl. 1, figs. 14-20.

Axoprunum angelinum (Campbell and Clark) Kling, 1973, p. 634, pl. 1 , figs. 13-16; pl. 6, figs. 14-18.

Genus HAECKELIELLA Hollande and Enjumet, 1960

Haeckeliella inconstans Dumitricǎ

(Plate 2, Figures 7, 8a, 8b)

Haeckeliella inconstans Dumitrică, 1973, p. 833, pl. 7, figs. 1, 2; pl. 18 , figs. $7-22$.

Remarks: The forms, which were obtained from Pliocene sediments cored during the present cruise, agree well with the type species. However, specimens from the Pleistocene sediments tend to have sharply bladed spines as viewed in cross section, and the tip of spines becomes less distinctly ramified.

Genus SPHAEROPYLE Dreyer, 1889

Sphaeropyle langii Dreyer

(Plate 2, Figures 3a, 3b, 3c)

Sphaeropyle langii Dreyer, 1889, p. 13, pl. 4, fig. 54; Foreman, 1975, p. 618 , pl. 9 , figs. 30,31 .

\section{Sphaeropyle robusta $\mathrm{Kling}$}

(Plate 2, Figures 4a, 4b, 4c)

Sphaeropyle robusta Kling, 1973, p. 634, pl. 1, figs. 11, 12; pl. 6, figs. 9-13, figs. 1-5; Foreman, 1975, p. 618, pl. 9, figs. 24-26.

\section{Genus STYLACONTARIUM Popofsky, 1912 \\ Stylacontarium acquilonium (Hays) \\ (Plate 2, Figures 2a, 2b)}

Druppatractus acquilonius Hays, 1970, p. 214, pl. 1, figs. 4, 5.

Stylacontarium acquilonium (Hays), Kling, 1973, p. 634, pl. 1, figs. 17-20; pl. 14, figs. 1-4.

\section{Genus THECOSPHAERA Haeckel, 1881 \\ Thecosphaera akitaensis Nakaseko \\ (Plate 2, Figures 6a, 6b)}

Thecosphaera akitaensis Nakaseko, 1972, p. 63, pl. 1, figs. 4a, 4b.

\section{Thecosphaera japonica Nakaseko}

(Plate 2, Figures 5a, 5b)

Thecosphaera japonica Nakaseko, 1972, p. 61, pl. 1, figs. 3a, 3b.

Remarks: I refer Pliocene specimens to this species' with confidence, but the younger forms, with short, slender spines, resemble Echinoma delicatulum (Dogiel) (Petrushevskaya, 1967, p. 18, fig. 11). I was unable satisfactorily to separate the early forms from the late. The latter seem to be conspecific with $E$. delicatulum, but I have treated them under the single name of Thecosphaera japonica in order to establish stratigraphic correlation with Japanese land sequences. 


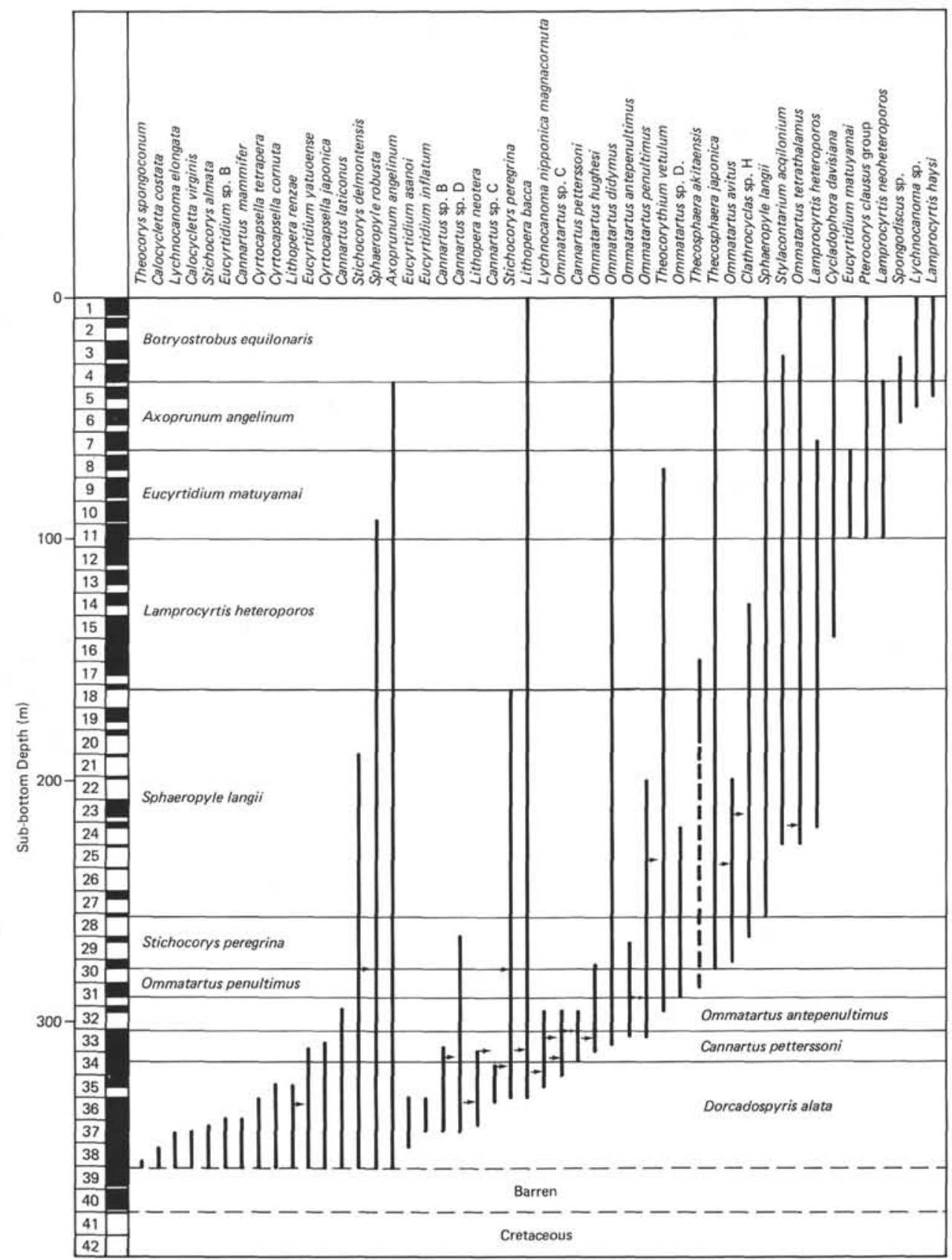

Figure 1. Radiolarian ranges and zones at Site 436. The broken line indicates the possible range of a species which was absent in the middle part of its range. A pair of arrows indicates a horizon of the evolutionary transition.

Subfamily ARTISCINAE Haeckel, 1881, emend. Riedel, 1967

I report 17 forms from Site 436 materials as belonging to this subfamily and assign 15 of them to four phylogenetic lineages (see Radiolarian Events). Most of these species are reported under tentative generic and specific names because they have not been compared closely with those forms reported by previous workers.

Genus CANNARTUS Haeckel, 1881, emend. Riedel, 1971

Cannartus laticonus Riedel

(Plate 3, Figures 7a, 7b, 8a, 8b)

Cannartus laticonus Riedel, 1959, p. 291, pl. 1, fig. 5 .

Remarks: This species is distinguished from its evolutionary descendant Ommatartus antepenultimus in not having pronounced caps but instead a parallel-sided clear zone of which width is less than 20 per cent of the cortical shell length (Westberg and Riedel, 1978).

\section{Cannartus mammifer (Haeckel) \\ (Plate 3, Figures 11a, 11b)}

Cannartidium mammiferum Haeckel, 1887, p. 375, pl. 39, fig. 16. Cannartus mammifer (Haeckel), Riedel, 1959, p. 291, pl. 1, fig. 4.

\section{Cannartus petterssoni Riedel and Sanfilippo}

(Plate 4, Figures 4a, 4b, 6a, 6b, 7a, 7b)

Cannartus (?) petterssoni Riedel and Sanfilippo, 1970, p. 520, pl. 14, fig. 3 . 
TABLE 6

Radiolarian Events at Sites 434, 435, and 436

\begin{tabular}{|c|c|c|c|c|c|c|c|c|}
\hline & \multirow[b]{2}{*}{ Radiolarian Events } & & \multicolumn{6}{|c|}{$\begin{array}{c}\text { Core Sections } \\
\text { (Depth in Meters) }\end{array}$} \\
\hline & & & \multicolumn{2}{|c|}{ Site 434} & \multicolumn{2}{|l|}{ Site 435} & \multicolumn{2}{|l|}{ Site 436} \\
\hline $\mathrm{T}$ & Spongodiscus sp. & $\mathrm{m}$ & & & $\begin{array}{l}2, \mathrm{CC} \\
4-1 \\
(16-27)\end{array}$ & G & $\begin{array}{l}2-3 \\
3, \mathrm{CC} \\
(12-25)\end{array}$ & G \\
\hline $\mathrm{T}$ & $\begin{array}{l}\text { Stylacontarium } \\
\text { acquilonium }\end{array}$ & e & $\begin{array}{l}1, C C \\
2-1 \\
(6-7)\end{array}$ & & $\begin{array}{l}2, \mathrm{CC} \\
4-1 \\
(16-27)\end{array}$ & G & $\begin{array}{l}2-3 \\
3, \mathrm{CC} \\
(12-25)\end{array}$ & M \\
\hline $\mathrm{T}$ & $\begin{array}{l}\text { Axoprunum } \\
\text { angelinum }\end{array}$ & e & $\begin{array}{l}1, \mathrm{CC} \\
2-1 \\
(6-7)\end{array}$ & & $\begin{array}{l}2, C C \\
4-1 \\
(16-27)\end{array}$ & G & $\begin{array}{l}4-3 \\
4-6 \\
(30-35)\end{array}$ & G \\
\hline B & Lychnocanoma sp. & m & $\begin{array}{l}1, \mathrm{CC} \\
2-1 \\
(6-7)\end{array}$ & & $\begin{array}{l}4, C C \\
5-1 \\
(32-37)\end{array}$ & G & $\begin{array}{l}6-1 \\
6-3 \\
(47-50)\end{array}$ & M \\
\hline B & Spongodiscus sp. & $\mathrm{m}$ & & & $\begin{array}{l}6-4 \\
6-5 \\
(51-52)\end{array}$ & G & $\begin{array}{l}6-5 \\
7-4 \\
(52-60)\end{array}$ & M \\
\hline $\mathrm{T}$ & $\begin{array}{l}\text { Eucyrtidium } \\
\text { matuyamai }\end{array}$ & e & $\begin{array}{l}1, C C \\
2-1 \\
(6-7)\end{array}$ & & $\begin{array}{l}6-2 \\
6-4 \\
(48-51)\end{array}$ & G & $\begin{array}{l}7-4 \\
7, \mathrm{CC} \\
(60-64)\end{array}$ & G \\
\hline $\mathrm{T}$ & $\begin{array}{l}\text { Theocorythium } \\
\text { vetulum }\end{array}$ & $\mathrm{m}$ & & & $\begin{array}{l}8, \mathrm{CC} \\
9-1 \\
(68-75)\end{array}$ & $\mathrm{P}$ & $\begin{array}{l}7, \mathrm{CC} \\
8, \mathrm{CC} \\
(64-72)\end{array}$ & G \\
\hline $\mathrm{T}$ & $\begin{array}{l}\text { Sphaeropyle } \\
\text { robusta }\end{array}$ & m & $\begin{array}{l}1, \mathrm{CC} \\
2-1 \\
(6-7)\end{array}$ & & $\begin{array}{l}8-1 \\
8, C C \\
(65-68)\end{array}$ & $P$ & $\begin{array}{l}9, \mathrm{CC} \\
10, \mathrm{CC} \\
(83-93)\end{array}$ & G \\
\hline $\mathrm{T}$ & $\begin{array}{l}\text { Pterocanium } \\
\text { prismatium }\end{array}$ & e & & & & & $\begin{array}{l}10, \mathrm{CC} \\
11-2 \\
(93-95)\end{array}$ & $\mathbf{P}$ \\
\hline B & $\begin{array}{l}\text { Eucyrtidium } \\
\text { matuyamai }\end{array}$ & m-e & $\begin{array}{l}2, \mathrm{CC} \\
3, \mathrm{CC} \\
(8-16)\end{array}$ & M & $\begin{array}{l}8 . \mathrm{CC} \\
9-1 \\
(68-75)\end{array}$ & G & $\begin{array}{l}11-5 \\
11, \mathrm{CC} \\
(100-103)\end{array}$ & G \\
\hline $\mathrm{B}^{\mathrm{a}}$ & $\begin{array}{l}\text { Pterocorys } \\
\text { clausus group }\end{array}$ & m & $\begin{array}{l}2-1 \\
2, \mathrm{CC} \\
(7-8)\end{array}$ & M & $\begin{array}{l}8, C C \\
9-1 \\
(68-75)\end{array}$ & G & $\begin{array}{l}11-5 \\
11, \mathrm{CC} \\
(100-103)\end{array}$ & M \\
\hline $\mathrm{T}$ & $\begin{array}{l}\text { Clathrocyclas } \\
\text { sp. H }\end{array}$ & m & $\begin{array}{l}5, C C \\
6, C C \\
(37-45)\end{array}$ & G & $\begin{array}{l}10, \mathrm{CC} \\
11-2 \\
(86-95)\end{array}$ & G & $\begin{array}{l}13-5 \\
14, \mathrm{CC} \\
(119-127)\end{array}$ & G \\
\hline $\mathrm{T}$ & $\begin{array}{l}\text { Thecosphaera } \\
\text { akitaensis }\end{array}$ & $\mathrm{m}$ & $\begin{array}{l}5, C C \\
6, C C \\
(37-45)\end{array}$ & G & $\begin{array}{l}10, \mathrm{CC} \\
11-2 \\
(86-95)\end{array}$ & M & $\begin{array}{l}15, \mathrm{CC} \\
16-7 \\
(141-150)\end{array}$ & M \\
\hline $\mathrm{B}^{\mathrm{a}}$ & $\begin{array}{l}\text { Cycladophora } \\
\text { davisiana }\end{array}$ & $\mathrm{m}$ & $\begin{array}{l}6, C C \\
7-1 \\
(45-54)\end{array}$ & M & $\begin{array}{l}11, \mathrm{CC} \\
12-1 \\
(98-104)\end{array}$ & G & $\begin{array}{l}15, \mathrm{CC} \\
16-7 \\
(141-150)\end{array}$ & M \\
\hline $\mathrm{T}$ & $\begin{array}{l}\text { Stichocorys } \\
\text { peregrina }\end{array}$ & e & $\begin{array}{l}6, C C \\
7-1 \\
(45-54)\end{array}$ & M & $\begin{array}{l}12, \mathrm{CC} \\
13-2 \\
(105-114)\end{array}$ & M & $\begin{array}{l}17-4 \\
18-2 \\
(156-162)\end{array}$ & G \\
\hline $\mathrm{T}$ & $\begin{array}{l}\text { Stichocorys } \\
\text { delmontensis }\end{array}$ & $\mathrm{m}$ & $\begin{array}{l}10, \mathrm{CC} \\
11, \mathrm{CC} \\
(83-92)\end{array}$ & $P$ & $\begin{array}{l}13-4 \\
13, \mathrm{CC} \\
(117-119)\end{array}$ & $\mathrm{P}$ & $\begin{array}{l}20, \mathrm{CC} \\
21-1 \\
(181-189)\end{array}$ & G \\
\hline $\mathrm{T}$ & $\begin{array}{l}\text { Ommatartus } \\
\text { sp. D }\end{array}$ & $\mathrm{m}$ & & & & & $\begin{array}{l}23, \mathrm{CC} \\
24, \mathrm{CC} \\
(215-220)\end{array}$ & M \\
\hline B & $\begin{array}{l}\text { Lamprocyrtis } \\
\text { heteroporos }\end{array}$ & m & & & $\begin{array}{l}\text { below } \\
435 \mathrm{~A}, 11, \mathrm{CC} \\
(241)\end{array}$ & & $\begin{array}{l}24, \mathrm{CC} \\
25, \mathrm{CC} \\
(220-227)\end{array}$ & M \\
\hline & $\begin{array}{l}\text { mmatartus avitus } \\
\text { O. tetrathalamus }\end{array}$ & & & & $\begin{array}{l}435 \mathrm{~A}, 10, \mathrm{CC} \\
435 \mathrm{~A}, 11, \mathrm{CC} \\
(227-241)\end{array}$ & P & $\begin{array}{l}24, \mathrm{CC} \\
25, \mathrm{CC} \\
(220-227)\end{array}$ & $\mathrm{P}$ \\
\hline B & $\begin{array}{l}\text { Stylacontarium } \\
\text { aquilonium }\end{array}$ & $\mathrm{m}$ & & & $\begin{array}{l}A, 10, C C \\
A, 11-1 \\
(227-235)\end{array}$ & $\mathrm{P}$ & $\begin{array}{l}25, \mathrm{CC} \\
26, \mathrm{CC} \\
(227-237)\end{array}$ & $\mathrm{M}$ \\
\hline $\mathrm{T}$ & $\begin{array}{l}\text { Lychnocanoma } \\
\text { nipponica } \\
\text { nipponica }\end{array}$ & $\mathrm{m}$ & & & & & $\begin{array}{l}26, \mathrm{CC} \\
27, \mathrm{CC} \\
(237-249)\end{array}$ & M \\
\hline & $\begin{array}{l}\text { mmatartus penultimus } \\
\text { O. avitus }\end{array}$ & & & & & & $\begin{array}{l}26, C C \\
29-1 \\
(237-265)\end{array}$ & M \\
\hline B & $\begin{array}{l}\text { Sphaeropyle } \\
\text { langii }\end{array}$ & $\mathrm{m}$ & & & & & $\begin{array}{l}28, \mathrm{CC} \\
29-1 \\
(256-265)\end{array}$ & M \\
\hline & $\begin{array}{l}\text { annartus sp. D } \\
\text { Ommatartus sp. D }\end{array}$ & & & & & & $\begin{array}{l}28, \mathrm{CC} \\
29-1 \\
(256-265)\end{array}$ & $\mathrm{M}$ \\
\hline$T$ & $\begin{array}{l}\text { Ommatartus } \\
\text { hughesi }\end{array}$ & $\mathrm{m}$ & & & & & $\begin{array}{l}29, \mathrm{CC} \\
30-2 \\
(267-276)\end{array}$ & $\mathrm{M}$ \\
\hline
\end{tabular}

TABLE 6 - Continued

\begin{tabular}{|c|c|c|c|c|c|c|}
\hline & & & & $\begin{array}{l}\text { Core Secti } \\
\text { Depth in Me }\end{array}$ & & \\
\hline & Radiolarian Events & & Site 434 & Site 435 & Site 436 & \\
\hline 1 & $\begin{array}{l}\text { Cannartus } \\
\text { sp. E }\end{array}$ & $\mathrm{m}$ & & & $\begin{array}{l}30-2 \\
30, \mathrm{CC} \\
(276-278)\end{array}$ & M \\
\hline & $\begin{array}{l}\text { a Thecosphaera } \\
\text { japonica }\end{array}$ & $\mathrm{m}$ & & & $\begin{array}{l}30, C C \\
31-1 \\
(278-284)\end{array}$ & M \\
\hline & $\begin{array}{l}\text { tichocorys } \\
\text { elmontensis } \\
\text { S. peregrina }\end{array}$ & & & & $\begin{array}{l}30, \mathrm{CC} \\
31-1 \\
(278-284)\end{array}$ & G \\
\hline & $\begin{array}{l}\text { Immatartus } \\
\text { ntepenultimus } \\
\text { O. penultimus }\end{array}$ & & & & $\begin{array}{l}31, C C \\
32-2 \\
(290-295)\end{array}$ & G \\
\hline 1 & $\begin{array}{l}\text { Lychnocanoma } \\
\text { nipponica } \\
\text { magnacornuta }\end{array}$ & $\mathrm{m}$ & & & $\begin{array}{l}31, C C \\
32-2 \\
(290-295)\end{array}$ & M \\
\hline & $\begin{array}{l}\text { annartus petterssoni } \\
\text { Ommatartus hughesi }\end{array}$ & & & & $\begin{array}{l}33-1 \\
33-3 \\
(306-307)\end{array}$ & G \\
\hline & $\begin{array}{l}\text { mmatartus sp. C } \\
\text { O. didymus }\end{array}$ & & & & $\begin{array}{l}33-3 \\
33-4 \\
(306-307)\end{array}$ & P \\
\hline 1 & $\begin{array}{l}\text { Cyrtocapsella } \\
\text { japonica }\end{array}$ & $\mathrm{m}$ & & & $\begin{array}{l}33-4 \\
33-5 \\
(307-309)\end{array}$ & M \\
\hline 1 & $\begin{array}{l}\text { Eucyrtidium } \\
\text { yatuoense }\end{array}$ & $\mathrm{m}$ & & & $\begin{array}{l}33-5 \\
33-6 \\
(309-310)\end{array}$ & M \\
\hline 1 & $\begin{array}{l}\text { Lithopera } \\
\text { neotera }\end{array}$ & $\mathrm{m}$ & & & $\begin{array}{l}33-6 \\
33, \mathrm{CC} \\
(310-312)\end{array}$ & G \\
\hline & $\begin{array}{l}\text { ithopera neotera } \\
\text { L. bacca }\end{array}$ & & & & $\begin{array}{l}33, \mathrm{CC} \\
34-1 \\
(312)\end{array}$ & G \\
\hline & $\begin{array}{l}\text { annartus sp. B } \\
\text { C. petterssoni }\end{array}$ & & & & $\begin{array}{l}34-2 \\
34-3 \\
(314-315)\end{array}$ & M \\
\hline $\mathrm{b}$ & $\begin{array}{l}\text { Cannartus } \\
\text { petterssoni }\end{array}$ & $\mathrm{m}$ & & & $\begin{array}{l}34-3 \\
34-4 \\
(315-317)\end{array}$ & G \\
\hline & $\begin{array}{l}\text { annartus sp. C } \\
\text { Ommatartus sp. C }\end{array}$ & & & & $\begin{array}{l}34-5 \\
34, C C \\
(318-320)\end{array}$ & $\mathbf{P}$ \\
\hline 1 & $\begin{array}{l}\text { Cyrtocapsella } \\
\text { cormuta }\end{array}$ & $\mathrm{m}$ & & & $\begin{array}{l}35-3 \\
35-4 \\
(325-326)\end{array}$ & P \\
\hline 1 & $\begin{array}{l}\text { Lithopera } \\
\text { renzae }\end{array}$ & $\mathrm{m}$ & & & $\begin{array}{l}35-3 \\
35-4 \\
(325-326)\end{array}$ & G \\
\hline $\mathrm{t}$ & $\begin{array}{l}\text { Lychnocanoma } \\
\text { nipponica } \\
\text { magnacornuta }\end{array}$ & m & & & $\begin{array}{l}35-4 \\
35, \mathrm{CC} \\
(326-327)\end{array}$ & $\mathrm{M}$ \\
\hline & $\begin{array}{l}\text { b Cyrtocapsella } \\
\text { tetrapera }\end{array}$ & $\mathrm{m}$ & & & $\begin{array}{l}35, \mathrm{CC} \\
36-1 \\
(327-331)\end{array}$ & G \\
\hline 1 & $\begin{array}{l}\text { Eucyrtidium } \\
\text { inflatum }\end{array}$ & $\mathrm{m}$ & & & $\begin{array}{l}35, \mathrm{CC} \\
36-1 \\
(327-331)\end{array}$ & M \\
\hline 1 & $\begin{array}{l}\text { Eucyrtidium } \\
\text { asanot }\end{array}$ & $\mathrm{m}$ & & & $\begin{array}{l}35, \mathrm{CC} \\
36-1 \\
(327-331)\end{array}$ & $P$ \\
\hline $\mathrm{E}$ & $\begin{array}{l}\text { Lithopera } \\
\text { bacca }\end{array}$ & $\mathrm{m}$ & & & $\begin{array}{l}36-1 \\
36-2 \\
(331-333)\end{array}$ & M \\
\hline & $\begin{array}{l}\text { ithopera renzae } \\
\text { L. neotera }\end{array}$ & & & & $\begin{array}{l}36-2 \\
36-4 \\
(333-336)\end{array}$ & G \\
\hline & $\begin{array}{l}\text { annartus sp. A } \\
\text { Cannartus sp. B }\end{array}$ & & & & $\begin{array}{l}36-4 \\
36-7 \\
(336-340)\end{array}$ & M \\
\hline 1 & $\begin{array}{l}\text { Eucyrtidium } \\
\text { sp. B }\end{array}$ & $\mathrm{m}$ & & & $\begin{array}{l}36-4 \\
36-7 \\
(336-340)\end{array}$ & $\mathbf{P}$ \\
\hline $\mathrm{b}$ & $\begin{array}{l}\text { Lychnocanoma } \\
\text { nipponica } \\
\text { nipponica }\end{array}$ & $\mathrm{m}$ & & & $\begin{array}{l}36-7 \\
37-2 \\
(340-343)\end{array}$ & M \\
\hline
\end{tabular}


TABLE 6 - Continued

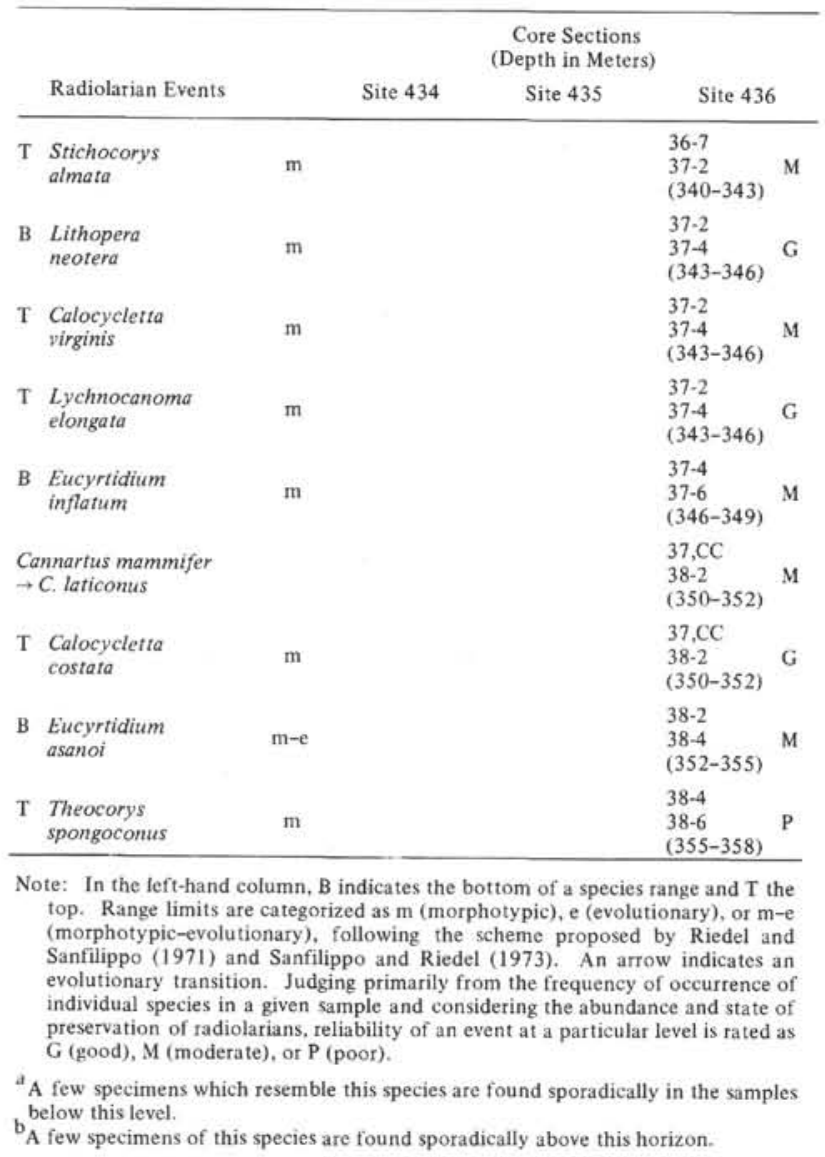

\section{Cannartus tubarius (Haeckel)}

(Plate 5, Figures 11a, 11b)

Pipettaria tubaria Haeckel, 1887, p. 339, pl. 39, fig. 15. Cannartus tubarius (Haeckel), Riedel and Sanfilippo, 1970, p. 520, pl. 15, fig. 2 .

Remarks: All specimens listed under this name have a rather thinwalled cortical shell with an indistinct equatorial constriction and one spherical inner and one lenticularly compressed outer medullary shell.

\section{Cannartus violina Haeckel}

(Plate 3, Figures 10a, 10b)

Cannartus violina Haeckel, 1887, p. 358, pl. 39, fig. 10; Riedel, 1959, p. 290 , pl. 1, fig. 3 .

Remarks: All specimens I identified with this species have a spherical or slightly ellipsoidal outer medullary shell.

\section{Cannartus sp. A}

(Plate 3, Figures 12a, 12b)

Remarks: Forms included here differ from Cannartus mammifer in having protuberances which cover the entire surface of the cortical shell rather than being limited to the broadest part. The younger forms tend to have indistinct protuberances at the equatorial part of the cortical shell. This cortical shell is small and cylindrical and is nearly the same shape as that of Cannartus $\mathrm{sp}$. B.

Cannartus sp. A has a broad, caplike clear zone between the cortical shell and spongy column. The ratio of zone height to cortical shell length ranges from 0.20 to 0.24 . This value falls within that of Ommatartus antepenultimus, but $O$. antepenultimus has a distinctly different cortical shell and its initial appearance is definitely earlier. Other artiscins which co-occur with Cannartus sp. A in the present materials, except for Cannartus sp. B, do not have such a broad clear zone.

\begin{tabular}{|c|c|}
\hline $\begin{array}{l}\text { This Chapter } \\
\text { (zone) }\end{array}$ & $\begin{array}{c}\text { Nakaseko and Sugano } \\
(1973)\end{array}$ \\
\hline Botryostrobus aquilonaris & \multirow{6}{*}{ Thecosphaera japonica } \\
\hline Axoprunum angelinum & \\
\hline Eucyrtidium matuyamai & \\
\hline Lamprocyrtis heteroporos & \\
\hline Sphaeropyle langii & \\
\hline Stichocorys peregrina & \\
\hline Ommatartus penultimus & \multirow{3}{*}{ Lychnocanium nipponicum } \\
\hline Ommatartus antepenultimus & \\
\hline Cannartus petterssoni & \\
\hline Dorcadospyris alata & Cyrtocapsella tetrapera \\
\hline
\end{tabular}

Figure 2. Comparison between the zonation used in this chapter and that previously established in Japanese land sequences.

The lower range of this species partly overlaps with $C$. mammifer and the upper range partly overlaps with Cannartus sp. B. Cannartus sp. A seems to be closely related to $C$. mammifer, but no transitional form was found in the samples examined here.

\section{Cannartus sp. B}

(Plate 4, Figures 9a, 9b)

Remarks: The cortical shell of this species is nearly cylindrical and sometimes bulges at the equator. In equatorial view, the two rows of protuberances on both ends of the cortical shell make a straight line. Spongy columns are subcylindrical and are as broad as the cortical shell. They are connected with the outer ring of protuberances at their bases. The first occurrence of this species is within the upper range of Cannartus sp. A and the last occurrence within the lower range of Cannartus petterssoni.

Cannartus sp. B closely resembles Cannartus sp. A and C. petterssoni but differs in having double rings formed by linear lined protuberances. Of these two rings the distal one becomes narrower in younger forms, and because of this the younger forms are difficult to distinguish from $C$. petterssoni. Cannartus $\mathrm{sp}$. B is judged to be the ancestor of $C$. petterssoni and a descendant of Cannartus sp. A.

\section{Cannartus sp. C}

(Plate 4, Figures 10a, 10b, 11a, 11b)

Remarks: The cortical shell of this species is distinctly constricted and is strongly plicated at the equator. Spongy columns are cylindrical and as broad as the cortical shell. Individual protuberances show a sign of irregular elongation and bear needle-shaped short spines which are arranged perpendicularly to the shell surface. The outer medullar 
shell is rather large, ellipsoidal or nearly cubic, and similar to that of Ommatartus didymus and Ommatartus sp. C. aff. O. didymus in shape and size.

The early form has rather conical spongy columns and a less plicated cortical shell and is difficult to distinguish from Cannartus laticonus. Therefore I have assigned the lowermost occurrence of this species somewhat arbitrarily. Further study is needed in order to determine distinguishing morphological characteristics.

Cannartus sp. C. differs from C. petterssoni and Cannartus sp. B in having a constricted cortical shell and from Ommatartus sp. C in having spongy columns which are not separated by any distinct dividing structure. It also differs from other artiscins in having broad spongy columns.

Cannartus sp. D

(Plate 5, Figures 7a, 7b, 8a, 8b, 9a, 9b, 10a, 10b)

Cannartus laticonus Riedel, Sanfilippo et al., 1973, p. 216, pl. 1, figs. 4-6.

Ommatartus antepenultimus Riedel and Sanfilippo, Sanfilippo et al., 1973, pl. 216, pl. 1, figs. 13-15.

Remarks: The forms recorded under this name occur commonly and consistently in the materials cored from Site 436 . They are comparable with both $C$. laticonus and $O$. antepenultimus reported from Mediterranian Neogene strata by Sanfilippo and Riedel (Sanfilippo et al., 1973). The early and later forms from this site are conspecific with $C$. laticonus and $O$. antepenultimus, respectively. However, I could not separate them stratigraphically because of their morphological continuity and the presence of transitional forms over a long stratigraphic interval. Thus they are grouped together under one species group.

This species is characterized by a robust cortical shell with a constriction at the equator and differs from the typical $C$. laticonus in having a large and thick-walled cortical shell. The earliest form observed in this material (Plate 5, Figures 10a, 10b) resembles Cannartiscus amphiconiscus Haeckel (Haeckel, 1887, p. 372, pl. 39, fig. 19) reported from the North Pacific, Station 253, but the latter has only one medullary shell.

Cannartus sp. E

(Plate 3, Figures 9a, 9b)

Ommatartus sp. cf. Cannartus bassani (Carnevale), Foreman, 1975, p. 619 , pl. 8 , figs. 9-12.

Remarks: The form recorded under this name is conspecific with that reported by Foreman (1975). This species seems to develop from C. laticonus or Cannartus sp. B, but its ancestor has not been determined because Cannartus sp. E occurred rarely at the lower part of its range.

\section{Cannartus spp.}

(Plate 5, Figures 2a, 2b, 5a, 5b, 6a, 6b)

Remarks: Under this name I recorded forms with an ellipsoidal cortical shell almost spherical in shape, lacking distinct constriction at the equator, and with spongy columns. Small pitlike furrows or slight depressions, which correspond to the plicated structure of species that do show constriction, are present at the equator. Each of the furrows or depressions joins to the cortical shell with a beam which connects the medullary shell. There may be several species within this group, but I have been unable to identify them satisfactorily. In the materials from Site 436 a rather similar form without spongy columns (Plate 5 , Figures $3 \mathrm{a}, 3 \mathrm{~b}$ ) occurs above the range of these forms.

Genus OMMATARTUS Haeckel, 1881, emend. Riedel, 1971

\section{Ommatartus antepenultimus Riedel and Sanfilippo}

(Plate 3, Figures 6a, 6b)

Ommatartus antepenultimus Riedel and Sanfilippo, 1970, p. 521, pl. 14 , fig. 14.

Remarks: In this chapter I have followed Westberg and Riedel (1978). Thus the ratio of polar cap height to cortical shell length is larger than 0.20 and less than 0.25 .
Ommatartus avitus (Riedel)

(Plate 3, Figures 2a, 2b)

Panartus avitus Riedel, 1953 , p. 808 , pl. 84, fig. 7.

Ommatartus avitus (Riedel), Riedel and Sanfilippo, 1971, p. 1588, pl. 4 , fig. 6 .

\section{Ommatartus didymus (Ehrenberg)}

(Plate 4, Figures 2a, 2b, 2c, 5ạ, 5b)

Haliomma didymus Ehrenberg, 1844, p. 83.

Ommatartus didymus (Ehrenberg), Sanfilippo et al., 1973, p. 216, pl. 2, figs. 1, 2 .

Remarks: The specimens that I assigned to this species have one or more polar caps, a slightly constricted cortical shell with a rough surface, and two medullary shells. The outer medullary shell is a large, nearly lenticular cube. In some specimens the paired polar caps extend to the equatorial part of the shell and form an additional cortical shell. The surface of the initial cortical shell is covered with numerous radial spines which connect with either the polar caps or the additional cortical shell. In the early form the height of the polar cap tends to be low, and the spine bases are broad and tend to fuse. Forms with protuberances, which occurred earlier than and together with the present species, I treat as a separate species under the name Ommatartus sp. C.

Some specimens reported as Ommatartus didymus and an allied form from near Tabiano in northern Italy (Sanfilippo et al., 1973, pl. 2 , figs. 2,3 ) resemble the early form from the present materials, but the form from the Torrente Rossi in Sicily (Sanfilippo et al., 1973, pl. 2 , fig. 1) was not found.

\section{Ommatartus hughesi (Campbell and Clark)}

(Plate 4, Figures 3a, 3b)

Ommatocampe hughesi Campbell and Clark, 1944, p. 23, pl. 3, fig. 12.

Ommatartus hughesi (Campbell and Clark), Riedel and Sanfilippo, 1970 , p. 521

Ommatartus penultimus (Riedel)

(Plate 3, Figures 3a, 3b, 4a, 4b, 5a, 5b)

Panarium penultimum Riedel, 1957, p. 76, pl. 1, fig. 1. Ommatartus penultimus (Riedel), Riedel and Sanfilippo, 1970, p. 521.

\section{Ommatartus tetrathalamus (Haeckel)}

(Plate 3, Figures 1a, 1b)

Panartus tetrathalamus Haeckel, 1887 , p. 378 , pl. 40 , fig. 3 .

Ommatartus tetrathalamus (Haeckel), Riedel and Sanfilippo, 1971, p. 1588 , pl. 1C, figs. 5-7.

\section{Ommatartus sp. C}

(Plate 4, Figures 8a, 8b)

Remarks: Under this name I included forms which are intermediate between Cannartus sp. $\mathrm{C}$ and Ommatartus didymus. The cortical shell is rather large and is constricted at the equator. The protuberances are well developed over the entire surface of the cortical shell and form narrow ridges. The spongy columns are as broad as the cortical shell and are divided into parallel, distinctly clear zones or caps.

This species differs from Cannartus petterssoni and $O$. hughesi in having a constricted cortical shell, from Cannartus sp. C in having polar caps or spongy columns which are divided into parallel zones, and from $O$. didymus in having protuberances on the cortical shell. Also, this species differs from other species of the Artinscinae in having broad, spongy columns.

\section{Ommatartus sp. D}

(Plate 5, Figures 1a, 1b, 4a, 4b)

Ommatartus sp., Foreman, 1975, p. 618 , pl. 8, figs. 17, 18.

Remarks: The forms recorded under this name have a large cortical shell strongly constricted at the equator, an outer medullary shell 
lenticularly compressed, and delicate polar caps with a narrow space. Although most of the specimens were without caps, the extreme spininess at the polar region of the cortical shell indicated that the caps had broken off.

Subfamily SATURNALINAE Deflandre, 1953

Genus SATURNALIS Haeckel, 1881, emend. Nigrini, 1967

Saturnalis circularis Haeckel

(Plate 6, Figure 14)

Saturnaris circularis Haeckel, 1887, p. 131; Nigrini, 1967, p. 25, pl. 1, fig. 9.

Family SPONGODISCIDAE Haeckel, 1862, emend. Riedel, 1967

Genus SPONGASTER Ehrenberg, 1860

Spongaster berminghami (Campbell and Clark)

(Plate 6, Figure 3)

Spongasteriscus berminghami Campbell and Clark, 1944, p. 30, pl. 5, figs. 1, 2.

Spongaster berminghami (Campbell and Clark), Sanfilippo and Riedel, 1973 , p. 524

\section{Spongaster tetras irregularis Nigrini}

(Plate 6, Figure 2)

Spongaster tetras irregularis Nigrini, 1967, p. 43, pl. 5, fig. 2.

\section{Spongaster tetras tetras Ehrenberg}

(Plate 6, Figure 1)

Spongaster tetras Ehrenberg, 1860, p. 833.

Spongaster tetras tetras Ehrenberg, Nigrini, 1967, p. 41, pl. 5, figs. $1 \mathrm{a}, 1 \mathrm{~b}$

\section{Genus SPONGODISCUS Ehrenberg, 1844}

Spongodiscus ambus Sanfilippo and Riedel (Plate 6, Figure 4)

Spongodiscus ambus Sanfilippo and Riedel, 1974, p. 1024, pl. 1, figs. $12-14$.

\section{Spongodiscus sp.}

(Plate 6, Figure 5)

Spongodiscus sp., Ling, 1973, p. 778, pl. 1, figs, 9, 10.

Remarks: Specimens observed during the present leg are conspecific with those reported from the Bering Sea, from high latitudes of the North Pacific (Ling, 1973), and from the Sea of Japan (Ling, 1975).

\section{Order NASSELLARIA Ehrenberg, 1875}

Suborder CYRTIDA Haeckel, 1862, emend. Petrushevskaya, 1971 Family THEOPERIDAE Haeckel, emend. Riedel, 1967

\section{Genus CLATHROCYCLAS Haeckel, 1881}

Clathrocyclas bicornis Hays

(Plate 6, Figures 9a, 9b, 10a, 10b, 11a, 11b)

Clathrocyclas bicornis Hays, 1965, p. 179, pl. 3, fig. 3.

\section{Clathrocyclas sp. $\mathbf{H}$}

(Plate 6, Figures 12a, 12b, 13a, 13b)

Description: Shell is campanulate, rather thin walled, and covered by a spongy mantle.

Cephalis is small and hemispherical and has small subcircular pores which are irregularly arranged and widely spaced. There are two short pyramidal horns. The one which continues to the apical spine is approximately at the apex and the other, which continues the vertical spine, protrudes obliquely.

Thorax is spiny and subdivided into two parts. The upper part is hemispherical to subconical and is twice as broad as the cephalis. It expands markedly in a distal direction and grades into the lower part with a distinct shoulder. The lower part is campanulate to subcylindrical, and nearly four times the width of the cephalis. There are from eight to nine transverse rows of circular to subcircular pores, arranged in a hexagonal pattern, on the thorax. There are from five to six small pores on the upper part of one half of a given circumference and seven to nine large pores on its lower part.

Abdomen expands distally. The pores are identical in shape and arrangement with those of the lower part of the thorax. Three transverse rows of pores are usually present. The base of the abdomen is not marked by any particular structure and terminates in spines so shaped as to indicate they would eventually form a complete ring. There is a distinct internal lumbar stricture with circular cross section between the thorax and abdomen. In some specimens, the lumbar region is draped distally by a thin plate of hyaline.

The spongy mantle extends from the basal part of the cephalis to the upper part of the abdomen. It attaches to the cephalis and abdomen directly and to the thorax by means of spines.

Remarks: This species closely resembles Clathrocyclas bicornis but differs from it in having small horns and a rather thin wall covered with a spongy mantle. It also differs from Theocalyptera bicornis (Popofsky) (Riedel, 1958, p. 240, pl. 4, fig. 4) in having small horns and a thorax which is divided into two parts. Clathrocyclas sp. $\mathrm{H}$ is similar to T. bicornis spongothorax Chen (Chen, 1975, p. 467, pl. 12, figs. 1-3) in having a spongy mantle but differs from it in having tiny horns and fewer pores on the equatorial half of the thorax. This species shows the same feature as that of the form originally illustrated as Anthocorys akitaensis by Nakaseko et al. (1965), Nakaseko (1969), and subsequently by Ling (1975), but those forms which have been described under the same name by Ling $(1971,1973)$ and Nakaseko and Sugano (1973) are considered to be a separate species.

Genus CYCLADOPHORA Ehrenberg, 1847b

Cycladophora davisiana Ehrenberg

(Plate 6, Figures 6, 7a, 7b, 8a, 8b)

Cycladophora (?) davisiana Ehrenberg, 1861, p. 297.

Cycladophora davisiana Ehrenberg, Petrushevskaya, 1967, p. 122, figs. 69,70 .

Genus CYRTOCAPSELLA Haeckel, 1887, emend. Sanfilippo and Riedel, 1970

Cyrtocapsella cornuta (Haeckel)

(Plate 8, Figures 8a, 8b)

Cyrtocapsa (Cyrtocapsella) cornuta Haeckel, 1887, p. 1513, pl. 78, fig. 9.

Cyrtocapsella cornuta (Haeckel), Sanfilippo and Riedel, 1970, p. 453, pl. 1, figs. 19, 20.

\section{Cyrtocapsella japonica (Nakaseko)}

(Plate 8, Figures 7a, 7b)

Eucyringium japonicum Nakaseko, 1963, p. 193, pl. 4, figs. 1-3.

Cyrtocapsella japonica (Nakaseko), Sanfilippo and Riedel, 1970, p. 452 , pl. 1, figs. 13-15.

\section{Cyrtocapsella tetrapera (Haeckel)}

(Plate 8, Figures 5, 6)

Cyrtocapsa (Cyrtocapsella) tetrapera Haeckel, 1887, p. 1512, pl. 78, fig. 5 .

Cyrtocapsella tetrapera (Haeckel), Sanfilippo and Riedel, 1970, p. 435 , pl. 1 , figs. $16-18$

\section{Genus EUCYRTIDIUM Ehrenberg, 1847a}

Eucyrtidium asanoi Sakai, new species

(Plate 7, Figures 12a, 12b, 13a, 13b, 13c, 14a, 14b)

Eucyrtidium sp., Ling, 1973, p. 781, pl. 2, fig. 9.

Description: Shell is spindle-shaped and thin walled. It is covered by longitudinal ridges which are made of thin hyaline plates and consists of five or more segments that are distinguished by internal lumbar strictures. There is an external constriction between the second (thoracic) and third (abdominal) segments that divides the shell into the cephalothoracic and postthoracic part. 
The cephalis is subspherical to hemispherical; has small, circular, widely spaced pores; and bears a tiny apical horn. Collar stricture is indistinct. Thorax is conically inflated, slightly rough surfaced, and bears hexagonally arranged circular pores. There is a very thin, porous plate (wing) on the thorax which continues to the internal spine at the proximal portion. Abdomen is truncate-conical in outline. Two or more post-abdominal segments are subcylindrical without externally expressed strictures. Thin-plated ridges are arranged longitudinally on the postthoracic part. Postthoracic pores are circular and are arranged in longitudinal rows. There are two or three pores between each pair of parallel ridges. The shell is widest around the fourth segment, with some variation occurring somewhere between the third and fifth segments.

Dimensions: Measurements are based on 12 specimens from Samples 436-36-4 $(35-37 \mathrm{~cm}), 436-36-7(25-27 \mathrm{~cm})$, and $436-37, \mathrm{CC}$ (13-15 cm). Width of cephalis is 15 to $20 \mu$; thorax, 45 to $55 \mu$; abdomen, 65 to $80 \mu$; fourth segment, 75 to $90 \mu$; and fifth segment, 75 to $80 \mu$. Maximum width of shell is 75 to $90 \mu$. Length of cephalis to the distal end of thorax is 40 to $45 \mu$; to end of abdomen, 80 to $95 \mu$; to end of the fourth segment, 110 to $125 \mu$; and to end of the fifth segment (on 7 specimens), 140 to $155 \mu$. Diameter of pores is 1 to $2 \mu$ on cephalis and 4 to $6 \mu$ on remaining segments.

Remarks: This species is distinguished from others of the genus by its longitudinal ridges separating rows of pores into sets.

This species is named in honor of Professor Kiyoshi Asano, who established a foundation for research in smaller foraminifera in the Far East and constantly encouraged my own research on radiolarians.

Holotype: Plate 7, Figures 13a, 13b, 13c (IGPS coll. cat. no. 73817) from Sample 436-36-4, 35-37 cm.

Eucyrtidium calvertense Martin group

(Plate 7, Figures 2a, 2b, 4a, 4b, 5a, 5b, 6a, 6b)

Eucyrtidium calvertense Martin, 1904, p. 450, pl. 130, fig. 5 .

Remarks: In this group I include forms with a thick-walled, spindle-shaped shell which is broadest at the fourth or fifth segment. A small thorax is marked off from postthoracic segments by a pronounced change in shell outline.

E. matuyamai is distinguished from this group by its larger size and deeper furrows.

There may be several species in this group, but I was unable to differentiate them satisfactorily.

\section{Eucyrtidium cienkowskii Haeckel group}

(Plate 7, Figures 8a, 8b, 9, 10)

Remarks: This species group is characterized by a small cephalothorax segment which is marked off from the remainder of the shell by a pronounced change in shell outline. The third segment is subconical and the remaining segments are subcylindrical. Pores of the third and subsequent segments tend to align longitudinally, and there are 14 to 20 pores on one half of a given circumference.

There may be more than one species in this group, but $\mathrm{I}$ have been unable to differentiate them satisfactorily.

\section{Eucyrtidium matuyamai Hays}

(Plate 7, Figures 1a, 1b)

Eucyrtidium matuyamai Hays, 1970, p. 213, pl. 1, figs. 7-9.

Eucyrtidium inflatum Kling

(Plate 7, Figures 11a, 11b)

Eucyrtidium inflatum Kling, 1973, p. 636, pl. 11, fig. 7, pl. 15, figs. 7-10.

\section{Eucyrtidium yatsuoense Nakaseko}

(Plate 7, Figures 3a, 3b)

Eucyrtidium yatsuoense Nakaseko, 1955, p. 110, pl. 10, figs. 1a, 1b.

Remarks: This species resembles very closely Lithocampe (Lithocampium) diploconus Haeckel (Haeckel, 1887, p. 1505, pl. 77, fig. 3).

\section{Eucyrtidium sp. B}

$$
\text { (Plate 7, Figures 7a, 7b) }
$$

Description: The form reported under this name has a spindleshaped shell which consists of four thick-walled segments. A tiny apical horn is present. An external stricture between the thorax and abdomen is well defined. Cephalis is subspherical and has small circular pores. Collar stricture is distinct. Thorax is inflated conically and bears circular pores arranged in a hexagonal pattern. From six to seven pores are arranged in each longitudinal row, with about 10 on one half of a given circumference. Abdomen is inflated-conical to truncate-fusiform, and the fourth segment is inverted truncate-conical in shape. Internal lumbar stricture is well defined, but there is no externally expressed stricture between the abdomen and fourth segment. These postthoracic segments have circular pores which are arranged in longitudinal rows and set in shallow furrows. There are from 10 to 12 abdominal pores in each longitudinal row and from 12 to 14 on one half of a given circumference.

Remarks: This species differs from Eucyrtidium inflatum in having only four segments, a rather large thorax, and a long abdomen with more pores per longitudinal row. It differs from $E$. yatsuoense in having a long abdomen and a distinct constriction between the thorax and abdomen. This species strongly resembles Phormocyrtis turgida (Krasheninnikov) (Foreman, 1973, p. 438, pl. 7, fig. 10, pl. 12, fig. 6 but differs from it in having larger pores on each longitudinal row.

Genus LITHOPERA Ehrenberg, 1847a

Lithopera bacca Ehrenberg

(Plate 8, Figure 9)

Lithopera bacca Ehrenberg, 1872a, p. 314; Nigrini, 1967, p. 54, pl. 6, fig. 2.

Lithopera neotera Sanfilippo and Riedel

(Plate 8, Figures 10, 11)

Lithopera neotera Sanfilippo and Riedel, 1970, p. 454, pl. 1, figs. $24-26,28$.

\section{Lithopera renzae Sanfilippo and Riedel}

(Plate 8, Figure 12)

Lithopera renzae Sanfilippo and Riedel, 1970, p. 454, pl. 1, figs. $21-23,27$.

Genus LYCHNOCANOMA Haeckel, 1887, emend. Foreman, 1973 Lychnocanoma elongata (Vinassa)

(Plate 9, Figure 4)

Tetrahedrina elongata Vinassa, 1900, p. 243, pl. 2, fig. 31.

Lychnocanoma elongata (Vinassa), Sanfilippo and Riedel, in Sanfilippo et al., 1973, p. 221, pl. 5, figs. 19, 20.

\section{Lychnocanoma nipponica magnacornuta Sakai, new subspecies}

(Plate 9, Figures 3a, 3b)

Lychnocanium nipponicum Nakaseko, Nakaseko and Sugano, 1973, pl. 3 , figs. 1a, 1b.

Lychnocanium sp., Ling, 1973, p. 781, pl. 2, figs. 10, 11.

Description: Shell of this subspecies is similar to that of

Lychnocanoma nipponica nipponica except that its apical horn is markedly large -40 to 70 per cent of the cephalis in width and 2.5 to 4.0 times the cephalis in length.

Dimensions: Measurements are based on 20 specimens from Samples $436-33-4,25$ to $27 \mathrm{~cm}$; 436-33-CC, 6 to $8 \mathrm{~cm} ; 436-34-2,45$ to $47 \mathrm{~cm}$; and 436-35-1, 50 to $52 \mathrm{~cm}$. Length of horn is 60 to $100 \mu$; cephalis, 20 to $25 \mu$; thorax, 85 to $95 \mu$; and foot, 130 to $180 \mu$. Width of horn is 15 to $25 \mu$; cephalis 35 to $45 \mu$; thorax 110 to $130 \mu$; and foot 20 to $25 \mu$. Diameter of pores in thorax is 8 to $15 \mu$.

Remarks: Although the forms reported by Ling (1973) from the subarctic region of the North Pacific, (DSDP Site 173) have a rather small thorax, they are considered a morphological variation of this subspecies.

Holotype: Plate 9, Figures 3a, 3b (IGPS coll. cat. no. 73818) from Sample 436-34-2, 45-47 cm.

\section{Lychnocanoma nipponica nipponica (Nakaseko)}

(Plate 9, Figures 2a, 2b)

Lychnocanium nipponicum Nakaseko, 1963, p. 168, text fig. 2, pl. 1, figs. $1 \mathrm{a}, 1 \mathrm{~b}$.

Remarks: Following Foreman (1973), the generic name Lychnocanoma is used for this species. 
Lychnocanoma sp.

(Plate 9, Figures 1a, 1b)

Description: Shell consists of two complete segments, three feet, and a fragile, generally incomplete third segment. Cephalis is hemispherical and has a few small, pitlike pores. Cephalis is adorned by a slender, conical horn whose base is ridged. Collar stricture is externally well defined. Thorax is hemispherical and bears circular pores arranged in a hexagonal pattern marked by distinct, high interpore ridges. Shell surface is rough with numerous tiny spines rising from each node of the frame. Three stout, slightly outward-flexed, threebladed feet extend downward from the distal end of the thorax. The third segment has a thin, fragile, irregularly pored wall which joins the three feet proximally. When the wall is broken away or not well developed, there are tiny thorns on the inner margin of the proximal feet and/or fragments of the third segment attached to the thorax.

Remarks: This species very closely resembles Lychnocanoma nipponica nipponica, except that the former has a small thorax and a third segment which directly joins the feet.

Genus LYCHNODICTUM Haeckel, 1881

\section{Lychnodictum audax Riedel}

(Plate 8, Figures 16a, 16b)

Lychnodictum audax Riedel, 1953, p. 810, pl. 85, fig. 9.

Remarks: Stratigraphically early forms from the present leg tend to have an elongate thorax and less distinctly latticed feet. Most of the specimens have a less thorny horn than that of the type specimen. In some specimens the thorns are reduced to small tubercles.

Genus PTEROCANIUM Ehrenberg, 1847a

Pterocanium prismatium Riedel

(Plate 8, Figures 15a, 15b)

Pterocanium prismatium Riedel, 1957, p. 87 , pl. 3, figs. 4, 5.

Genus STICHOCORYS Haeckel, 1881

\section{Stichocorys almata (Haeckel)}

(Plate 8, Figures 4a, 4b)

Cyrtophormis almata Haeckel, 1887, p. 1460, pl. 78, fig. 17.

Stichocorys almata (Haeckel), Riedel and Sanfilippo, 1971, p. 1595, pl. 2E, figs. 13-15.

\section{Stichocorys delmontensis (Campbell and Clark)}

(Plate 8, Figure 3)

Eucyrtidium delmontense Campbell and Clark, 1944, p. 56, pl. 7, figs. 19, 20.

Stichocorys delmontensis (Campbell and Clark), Sanfilippo and Riedel, 1970 , p. 451 , pl. 1 , fig. 9

\section{Stichocorys peregrina (Riedel) \\ (Plate 8, Figures 1, 2)}

Eucyrtidium elongatum peregrinum Riedel, 1953, p. 812 , pl. 85 , fig. 2.

Stichocorys peregrina (Riedel), Sanfilippo and Riedel, 1970, p. 451, pl. 1, fig. 10.

Genus THEOCORYS Haeckel, 1881

Theocorys redondoensis (Campbell and Clark)

(Plate 8, Figure 13)

Theocyrtis redondoensis Campbell and Clark, 1944, p. 49, p. 7, fig. 4 .

Theocorys redondoensis (Campbell and Clark), Kling, 1973, p. 638, pl. 11, figs. 26-28.

\section{Theocorys spongoconum Kling}

(Plate 8, Figure 14)

Theocorys spongoconum Kling, 1971, p. 1087, pl. 5, fig. 6 .

Family PTEROCORYIDAE Haeckel, 1881, emend. Riedel, 1967

Genus ANTHOCYRTIDIUM Haeckel, 1881

\section{Anthocyrtidium angulare Nigrini}

(Plate 9, Figure 7)

Anthocyrtidium angulare Nigrini, 1971, pl. 34.1, figs. 3a, 3b.

Genus CALOCYCLETTA Haeckel, 1887

Calocycletta costata (Riedel)

(Plate 10, Figures 6a, 6b)

Calocyclas costata Riedel, 1959, p. 296, pl. 2, fig. 9.

Calocycletta costata (Riedel), Riedel and Sanfilippo, 1970, p. 535, pl. 14, fig. 12.

\section{Calocycletta virginis Haeckel}

(Plate 10, Figures 5a, 5b)

Calocyclas (Calocycletta) virginis (Haeckel, 1887, p. 1381, pl. 74, fig. 4 .

Calocycletta virginis Haeckel, Moore, 1972, p. 147, pl. 1, fig. 7.

Genus LAMPROCYRTIS Kling, 1973

\section{Lamprocyrtis haysi Kling}

(Plate 9, Figures 8a, 8b)

Lamprocyrtis haysi Kling, 1973, p. 639, pl. 5, figs. 15, 16; pl. 15, figs. 1-3.

\section{Lamprocyrtis heteroporos (Hays)}

(Plate 9, Figures 10, 11a, 11b)

Lamprocyclas heteroporos Hays, 1965, p. 179, pl. 3, fig. 1.

Lamprocyrtis heteroporos (Hays), Kling, 1973, p. 639, pl. 5, figs. 19, 20.

\section{Lamprocyrtis neoheteroporos Kling}

(Plate 9, Figures 9a, 9b)

Lamprocyrtis neoheteroporos Kling, 1973, p. 639, pl. 5, figs. 17, 18; pl. 15 , figs. 4,5 .

Genus PTEROCORYS Haeckel, 1881, emend. Petrushevskaya, 1971

\section{Pterocorys clausus (Popofsky) group}

(Plate 9, Figures 5, 6a, 6b)

Lithornithium clausum Popofsky, 1913, p. 393, fig. 116.

Pterocorys clausus (Popofsky) group, Petrushevskaya and Kozlova, 1972 , p. 545 , pl. 36 , figs. $16-18$.

\section{Genus THEOCORYTHIUM Haeckel, 1887}

Theocorythium trachelium dianae (Haeckel)

(Plate 10, Figures 1a, 1b)

Theocorys dianae Haeckel, 1887, p. 1416, pl. 69, fig. 11.

Theocorythium trachelium dianae (Haeckel), Nigrini, 1967, p. 77, pl. 8, figs. 1a, 1b; pl. 9, figs. 1a, 1b.

\section{Theocorythium trachelium trachelium (Ehrenberg)}

(Plate 10, Figures 2a, 2b)

Eucyrtidium trachelius Ehrenberg, 1872a, p. 312.

Theocorythium trachelium trachelium (Ehrenberg), Nigrini, 1967, p. 79 , pl. 8 , fig. 2 ; pl. 9 , fig. 2 .

\section{Theocorythium vetulum Nigrini \\ (Plate 10, Figures 3a, 3b)}

Theocorythium vetulum Nigrini, 1971, p. 447, pl. 34.1 , figs. $6 \mathrm{a}, 6 \mathrm{~b}$.

Remarks: Stratigraphically early forms of this species tend to have a rather thick wall, short thorax, and paired cephalic lobes more laterally placed than those of stratigraphically later forms. The early forms closely resemble Lamprocyclas maritalis polypora Nigrini (Nigrini, 1967, p. 76, pl. 7, fig. 6).

Suborder SPYRIDA Ehrenberg, 1847a, emend. Petrushevskaya, 1971 Family TRIOSPYRIDAE Haeckel, 1881, emend. Petrushevskaya, 1971 
Genus DORCADOSPYRIS Haeckel, 1881

Dorcadospyris alata (Riedel)

(Plate 10, Figure 7)

Brachiospyris alata Riedel, 1959, p. 293, pl. 1, figs. 11, 12.

Dorcadospyris alata (Riedel), Riedel and Sanfilippo, 1970, p. 523, pl. 14 , fig. 5

\section{Dorcadospyris dentata Haeckel}

(Plate 10, Figure 8)

Dorcadospyris dentata Haeckel, 1887, p. 1040, pl. 85, fig. 6; Riedel, 1957 , p. 79 , pl. 1, fig. 4 .

\section{Dorcadospyris cf. Dorcadospyris dentata Haeckel} (Plate 10, Figures 9, 10)

Remarks: The form reported here is similar to Dorcadospyris dentata except that it lacks an apical horn. In some specimens the feet diverge at an angle of nearly $180^{\circ}$. This form is probably transitional from $D$. dentata to $D$. alata.

\section{ACKNOWLEDGMENTS}

I appreciate the constructive reviewing efforts of professors T. Saito of Yamagata University and K. Nakaseko of Osaka University. Thanks are due Miss U. Hino for her assistance in the final preparation of the manuscript.

\section{REFERENCES}

Campbell, A. S., and Clark, B. L., 1944. Miocene radiolarian faunas from Southern California. Geol. Soc. Am., Spec. Papers, 51, 76.

Chen, P. H., 1975. Antarctic Radiolalia. In Hayes, D. E., Frakes, L. A., et al., Init. Repts. DSDP, 28: Washington (U.S. Govt. Printing Office), 437-513.

Deflandre, G., 1953. Radiolaires fossiles. In Grasse, P. P., (Ed.), Traite de zoologie (Vol. 1, Pt. 2): Paris (Masson), 389-436.

Dreyer, F., 1889. Morphologische Radiolarienstudien, Die Pylombildungen in vergleichend-anatomischer und entwicklungsgeschichtlicher Beziehung bei Radiolarien und bei Protisten überhaupt, nebst System und Beschreibung neuer und der bis jetzt bekannten polymaticshen Spumellarien. Jena. Z Naturwiss., 23 (new ser. 16), 1-138.

Dumitricà, P., 1973. Cretaceous and Quaternary Radiolaria in deep sea sediments from the Northwest Atlantic Ocean and Mediterranean Sea. In Ryan, W. B. F., Hsü, K. J., et al., Init. Repts. DSDP, 13: Washington (U.S. Govt. Printing Office), 829-901.

Ehrenberg, C. G., 1838. Über die Bildung der Kreidefelsen und des Kreidemergels durch unsichtbare Organismen. $\mathrm{Kgl}$. Akad. Wiss. Berlin, Abhandl., 1838, 59-147.

1844. Über 2 neue Lager von Gebirgsmassen aus Infusorien als Meeres-Absatz in Nord-Amerika und eine Vergleichung derselben mit den organischen Kreide-Gebilden in Europa und Africa. Kgl. Preuss. Akad. Wiss. Berlin, Monatsber., 1844, 57-97.

1847a. Über eine halibiolithische, von Herrn R. Schomburgk entdeckte, vorherrschend aus mikroskopischen Polycystinen gebildete, Gebirgsmasse von Barbados. Kgl. Preuss. Akad. Wiss. Berlin, Monatsber., 1846, 382385.

1847b. Über die mikroskopischen kieselschaligen Polycystinen als machtige Gebirgsmasse von Barbados und über das Verhaltniss der aus mehr als 300 Neuen Arten bestehenden ganz eigenthumlichen Formengruppe jener Felsmasse zu den jetzt lebenden Thieren und zur Kreidebildung. Eine neue Anregung zur Erforschung des Erdlebens. Kgl. Preuss. Akad. Wiss, Berlin, Monatsber., 1847, 40-60. 1860. Über den Tiefgrund des stillen Oceans Zweischen Californien und den Sandwich-Inseln aus bis $15600^{\prime}$, Tiefe nach Lieut. Brookes. Kgl. Preuss. Akad. Wiss. Berlin, Monatsber., 1860, 819-833.

1861. Über die Tiefgrund-Verhaltnisse des Oceans am Eingange der Davisstrasse und bei Island. Kgl. Preuss. Akad.Wiss. Berlin, Monatsber., 1861, 275-315. 1872a. Mikrogeologischen Studien als Zusammenfassung der Beobachtungen des kleinsten Lebens der Meeres Tiefgrunde aller Zonen und-dessen geologischen Einfluss. Kgl. Preuss. Akad. Wiss. Berlin, Monatsber., $1872,265-322$.

1872a. Mikrogiologischen Studien über das Kleiste Leben der Meeres-Tiefgrunde aller Zonen und dessen geologischen Einfuss. Kgl. Akad. Wiss. Berlin, Abhandl., 1872, 131-399.

1875. Fortsetzung der mikrogiologischen Studien als Gesammt-Üebersicht der mikroskopischen Paläontologie gleichartig analysirter Gebirgsarten der Erde, mit specieller Rucksicht auf den Polycystinen-Mergel von Barbados. Kgl. Akad. Wiss. Berlin, Abhandl., 1875, 1-225.

Foreman, H. P., 1973. Radiolaria from DSDP Leg 20. In Heezen, B. C., MacGregor, I. D., et al., Init. Repts. DSDP, 20: Washington (U.S. Govt. Printing Office), 249-305.

1975. Radiolaria from the North Pacific, Deep Sea Drilling Project, Leg 32. In Larson, R. L., Moberly, R., et al., Init. Repts. DSDP, 32: Washington (U.S. Govt. Printing Office), 579-701.

Haeckel, E., 1862. Die Radiolarien: Berlin (Reimer). 1881. Entwurf eines Radiolarien-Systems auf Grund von Studien der Challenger-Radiolarien. Jena. Z. Med. Naturwiss., 15 (new ser. 8), 418-472.

1887. Report on the Radiolaria collected by H.M.S. Challenger during the years 1873-76. Rept. Voyage Challenger, Zool., 18, 1803.

Hays, J. D., 1965. Radiolaria and late Tertiary and Quaternary history of Antarctic seas. In Llano, G. A. (Ed.), Biology of Antarctic Seas II. Antarctic Research Ser. 5: Washington (Am. Geophys. Union), 124-184. 1970. Stratigraphy and evolutionary trends of Radiolaria in North Pacific deep-sea sediments. In Hays, J. D. (Ed.), Geological investigations of the North Pacific: Geol. Soc. Am., Mem., No. 126, 185-218.

Hays, J. D., and Shackleton, 1976. Globally synchronous extinction of the radiolarian Stylatractus universus. Geology, 4, 649-652.

Hollande, A., and Enjumet, M., 1960. Cytologie, evolution et systematique des Sphaerosides (Radiolaires). Archiv. Mus. Nat. Hist. Natur., 2, Ser. 7, 1-64.

Kling, S. A., 1971. Radiolaria: Leg 6 of the Deep Sea Drilling Project. In Fischer, A. G., Heezen, B. C., et al., Init. Repts. DSDP, 6: Washington (U.S. Govt. Printing Office), 1069-1117.

1973. Radiolaria from the eastern North Pacific, Deep Sea Drilling Project, Leg 18. In Kulm, L. D., von Huene, R., et al., Init. Repts. DSDP, 18: Washington (U.S. Govt. Printing Office), 617-671.

Ling, H. Y., 1971. Silicoflagellates and Ebridians from the Shinzan Diatomaceous Mudstone Member of the Onnagawa Formation (Miocene), Northeast Japan, In Farinacci, A. (Ed.), Plankt. Conf., 2nd, Roma 1970, Proc.: Rome (Tecnoscienza), 2, 689-703.

1973. Radiolaria: Leg 19 of the Deep Sea Drilling Project. In Creager, J. S., Scholl, D. W., et al., Init. Repts. DSDP, 19: Washington (U.S. Govt. Printing Office), 777-797.

1975. Radiolaria: Leg 31 of the Deep Sea Drilling Project. In Karig, D. E., Ingle, J. C., Jr. et al., Init. Repts. 
DSDP, 31: Washington (U.S. Govt. Printing Office), 703-761.

Martin, G. C., 1904. Radiolaria. Miocene: Baltimore (Maryland Geol. Survey), pp. 447-459.

Moore, T. C., Jr., 1972. Mid-Tertiary evolution of the radiolarian genus Calocycletta. Micropaleontology, 18, 144-152.

Müller, J., 1858. Uber die Thalassicollen, Polycystinen und Acanthometren des Mittelmeeres. Kgl. Akad. Wiss. Berlin, Abhandl., 1858, 1-62.

Nakaseko, K., 1955. Miocene radiolarian fossil assemblage from the southern Toyama Prefecture in Japan. Osaka Univ., South and North Coll., Sci Rept., 4, 65-140. 1963. Neogene Cyrtoidea (Radiolaria) from the Isozaki Formation in Ibaraki Prefecture, Japan. Osaka Univ., Coll. General Education, Sci. Rept., 12, 165-198. 1969. Neogene Radiolaria in Japan. First Internat. Conf. Planktonic Microfossils, Proc., 2, 468-474.

1972. On some species of the genus Thecosphaera from the Neogene formations, Japan. Osaka Univ., Coll. General Education, Sci. Rept., 20, 59-70.

Nakaseko, K., Iwamoto, H., and Takahashi, K., 1965. Radiolarian stratigraphy in the oil and gas bearing Tertiary and Upper Cretaceous formations, Japan. Economic Commission for Asia and Far East, 3rd Petrol. Symp., Tokyo, Japan, pp. 1-14.

Nakaseko, K., and Sugano, K., 1973. Neogene radiolarian zonation in Japan. Geol. Soc. Japan, Mem., No. 8, 23-34.

Nigrini, C. A., 1967. Radiolaria in pelagic sediments from the Indian and Atlantic Oceans. Univ. California, Scripps Inst. Ocean., Bull., 11, 1-125.

1971. Radiolarian zones in the Quaternary of the equatorial Pacific Ocean. In Funnell, B. M., and Riedel, W. R. (Eds.), The Micropaleontology of the Ocean: Cambridge (Cambridge University Press), pp. 443-461.

Oda, M., and Sakai, T., 1977. Microbiostratigraphy of the lower to middle part of the Hatatate Formation, Sendai, Japan. Prof. Kazuo Huzioka Memorial Volume: Akita (Fuzioka Kazuo Kyoju Taikan Kinen Kai), pp. 441-465.

Petrushevskaya, M. G., 1967. Radiolarians of Orders Spumellaria and Nassellaria of Antarctic region (from materials of Soviet Antarctic Expedition). In Biological Reports of the Soviet Antarctic Expedition (1955-1958) (Vol. 3): Leningrad (Nauka), 5-186.

1971. On the natural system of Polycystine Radiolaria (Class Sarcodina). In Farinacci, A. (Ed.), Plankt. Conf., 2nd, Roma 1970, Proc.: Rome (Tecnoscienza), 981-992.

Petrushevskaya, M. G., and Kozlova, G. E., 1972. Radiolaria: Leg 14, Deep Sea Drilling Project. In Hayes, D. E., Pimm, A. C., et al., Init. Repts. DSDP, 14: Washington (U.S. Govt. Printing Office), 495-648.
Popofsky, A., 1912. Die Sphaerellarien des Warmwassergebietes der Deutschen Sudopolar-Expedition, 1901-1903. Deutsche Sudopolar-Exped., 13 (Zool. 5), No. 2, 73-159. 1913. Die Nassellarien des Warmwasser-gebietes. Deutsche Sudopolar-Exped. 14 (Zool. 6), No. 2, 217-416.

Riedel, W. R., 1953. Mesozoic and Late Tertiary Radiolaria of Rotti. J. Paleont., 27, 805-813. 1957. Radiolaria: A preliminary stratigraphy. Rept. Swedish Deep-Sea Exped., 6, 59-96. 1958. Radiolaria in Antarctic sediments. Rept. B.A.N.Z. Antarctic Rec. Exped., 6, Ser. B, 213-255. 1959. Oligocene and Lower Miocene Radiolaria in tropical Pacific sediments. Micropaleontology, 5, 285-302. 1967. Class Actinopoda. In Harland, W. B., et al. (Eds), The Fossil Record: London (Geological Society of London), pp. 291-298. 1971. Systematic classification of polycystine Radiolaria. In Funnell, B. M., and Riedel, W. R. (Eds.), The Micropaleontology of the Ocean: Cambridge (Cambridge University Press), pp. 649-661.

Riedel, W. R., and Foreman, H. P., 1961. Type specimens of North American Paleozoic Radiolaria. J. Paleont., 35, 628-635.

Riedel, W. R., and Sanfilippo, A., 1970. Radiolaria, Leg 4, Deep Sea Drilling Project. In Bader, R. G., Gerard, R. D., et al., Init. Repts. DSDP, 4: Washington (U.S. Govt. Printing Office), 503-575. 1971. Cenozoic Radiolaria from the western tropical Pacific, Leg 7. In Winterer, E. L., Riedel, W. R., et al., Init. Repts. DSDP, 7, Pt. 2: Washington (U.S. Govt. Printing Office), 1529-1672. 1978. Stratigraphy and evolution of tropical Cenozoic radiolarians. Micropaleontology, 24, 61-96.

Sanfilippo, A., Burckle, L. H., Martini, E., and Riedel, W. R., 1973. Radiolarians, diatoms, silicoflagellates and calcareous nannofossils in the Mediterranean Neogene. Micropaleontology, 19, 209-234.

Sanfilippo, A., and Riedel, W. R., 1970. Post Eocene "closed" theoperid radiolarians. Micropaleontology, 16, 446-462. 1973. Cenozoic Radiolaria (exclusive of theoperids, artostrobiids and amphipyndacids) from the Gulf of Mexico, DSDP Leg 10. In Worzel, J. L., Bryant, W., et al., Init. Repts. DSDP, 10: Washington (U.S. Govt. Printing Office), 475-611.

1974. Radiolaria from the west-central Indian Ocean and Gulf of Aden, DSDP Leg 24. In Fisher, R. L., Bunce, E. T., et al., Init. Repts. DSDP, 24: Washington (U.S. Govt. Printing Office), 997-1035.

Vinassa de Regny, P. E., 1900. Radiolari Miocenici. Reale Accad. Sci. Ist Bologna, Mem., 8, Ser. 5, 565-595.

Westberg, M. J., and Riedel, W. R., 1978. Accuracy of radiolarian correlations in the Pacific Miocene. Micropaleontology, 24, 1-23. 


\section{PLATE 1}

All figured specimens are from Sample 436-41-1, 31-32 cm. Unless otherwise indicated, magnifications are $\times 200$.

Figure 1 Spongosaturnalis hueyi (Pessagno) group. S1-1, $\mathrm{K} 33 / 0(\times 125)$.

Figures 2, 3 Dictyomitra pseudomacrocephala Squinabol.

2. Sl-3, 031/0.

3. Sl-1, D60/4.

Figures 4a, b Protunuma (?) sp. Sl-2, F31/0.

Figures 5a, b Holocryptocanium barbui Dumitricǎ. Sl-3, W59/4.

Figures 6a, b Cryptamphorella conara (Foreman). Sl-1, F52/2.

Figure $7 \quad$ Lithocampe elegantissima Cita. Sl-1, V52/4.

Figure $8 \quad$ Lithomelissa (?) petila Foreman. Sl-1, R44/0.

Figure 9 Dictyomitra sp. cf. D. tekschaensis Aliev. Sl-1, $\mathrm{H} 60 / 0$.

Figures 10a- Hemicryptocapsa prepolyhedra Dumitricǎ. Sl-1, 10c Q39/4 $(10 \mathrm{a}, 10 \mathrm{~b}: \times 125)$.

Figure $11 \quad$ Dictyomitra sp. Sl-3, R52/2. 


\section{PLATE 1}
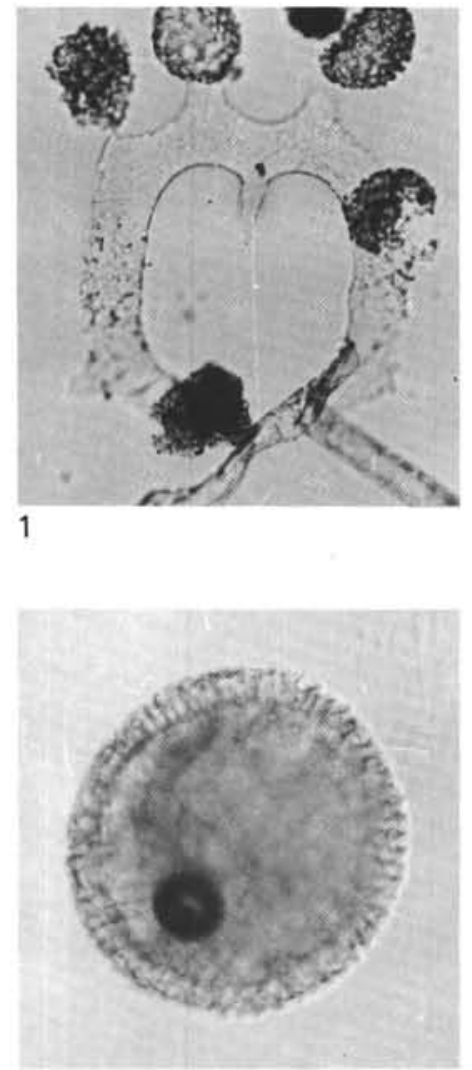

$5 a$

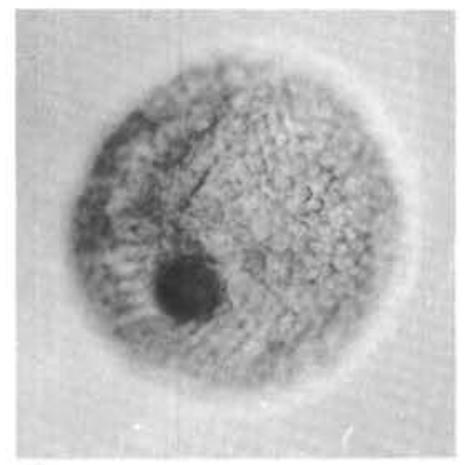

$5 b$

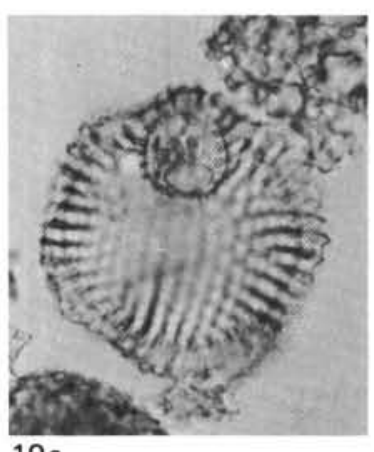

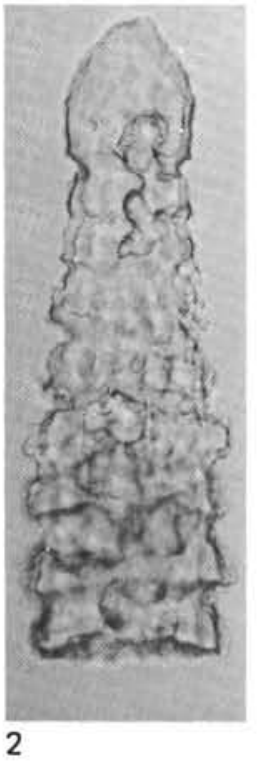

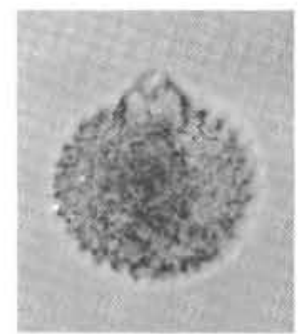

$6 a$

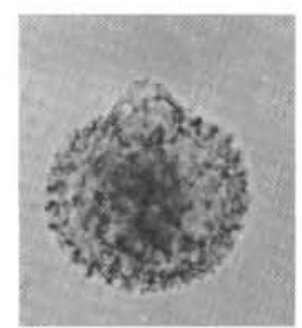

$6 b$

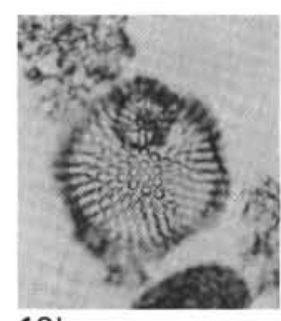

$10 \mathrm{~b}$
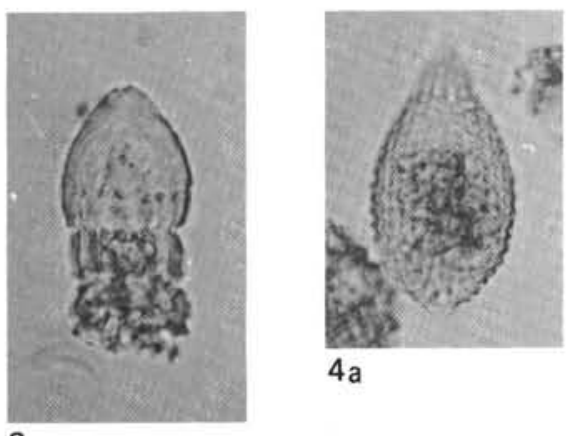

$4 \mathrm{a}$

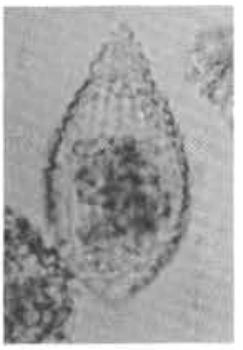

3

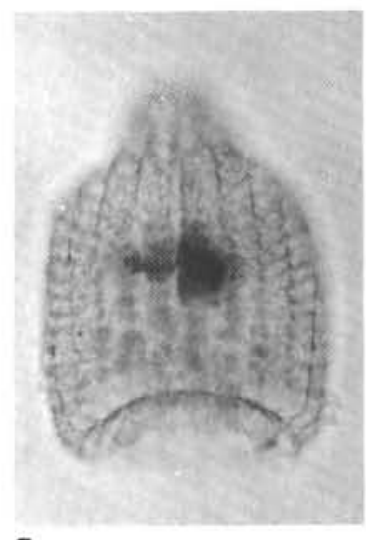

7

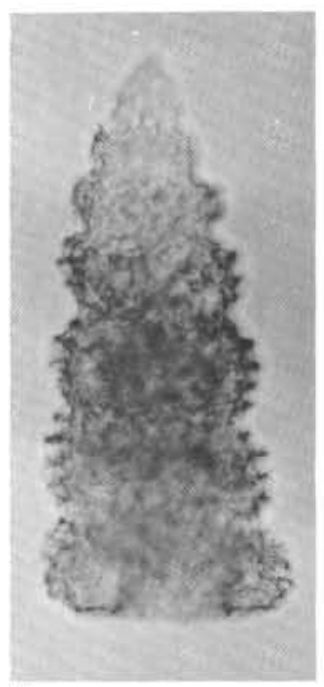

9

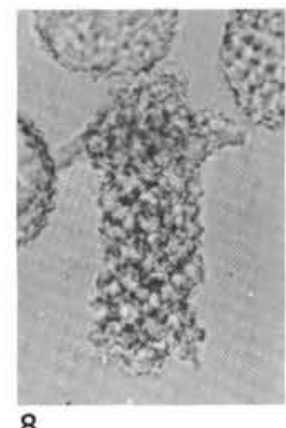

8

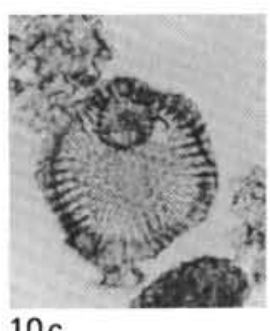

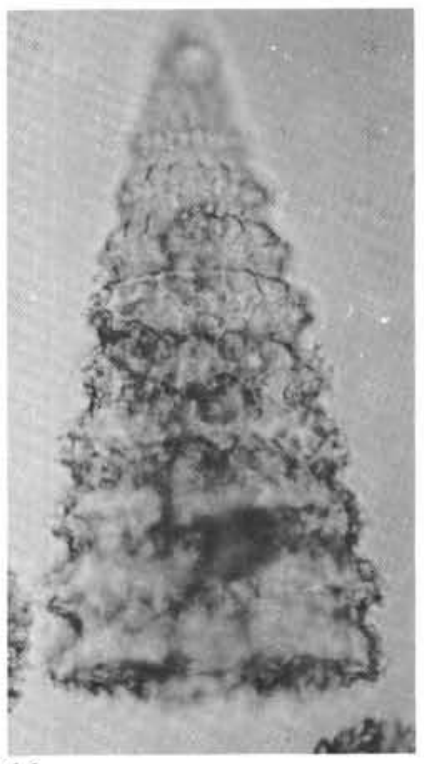

11 
PLATE 2

Unless otherwise indicated, magnifications are $\times 200$.

Figures 1a, b Axoprunum angelinum (Campbell and Clark). 434-2-1, 15-17 cm, Sl-1, K48/3.

Figures 2a, b Stylacontarium acquilonium (Hays). 436-3,CC, 0-2 cm, Sl-1, Q32/1.

Figures 3a-c Sphaeropyle langii Dreyer. 436-8,CC, 15-17 $\mathrm{cm}, \mathrm{Sl}-1, \mathrm{R} 23 / 3$.

Figures 4a-c Sphaeropyle robusta Kling. 436-38,CC, 10-12 cm, Sl-1, L60/0.

Figures 5a, b Thecosphaera japonica Nakaseko. 436-8,CC, 15-17 cm, S1-2, Y26/4.

Figures $6 \mathrm{a}, \mathrm{b}$ Thecosphaera akitaensis Nakaseko. 436-18-2, 57-59 cm, Sl-1, Q38/4.

Figures 7-8b Haeckeliella inconstans Dumitricǎ.

7. 436-13-5, 70-72 cm, Sl-2, J46/0 ( $\times 155)$.

8. 436-13-5, 70-72 cm, Sl-1, T26/1 ( $\times 155)$. 
PLATE 2
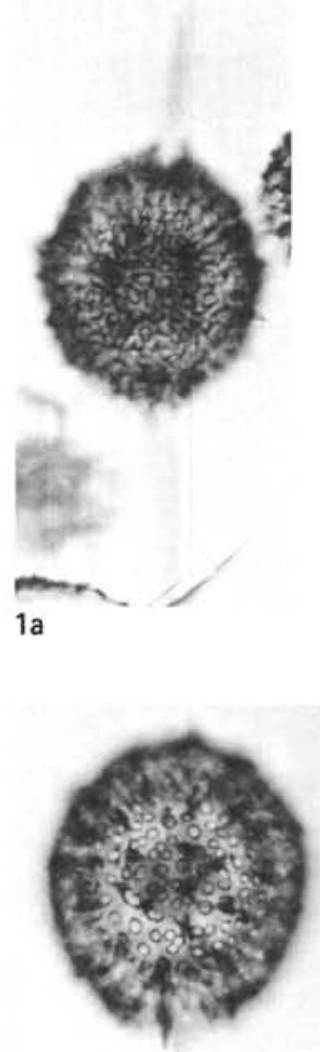

3a

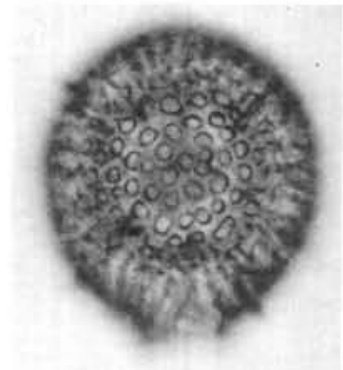

$4 a$

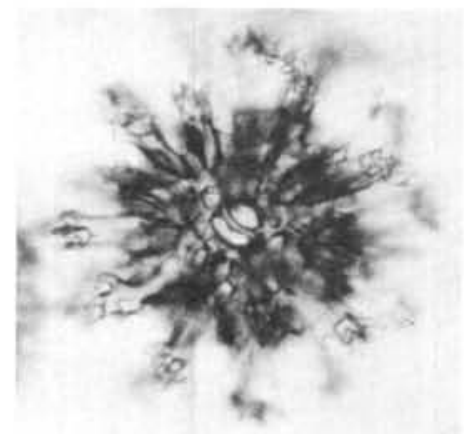

7
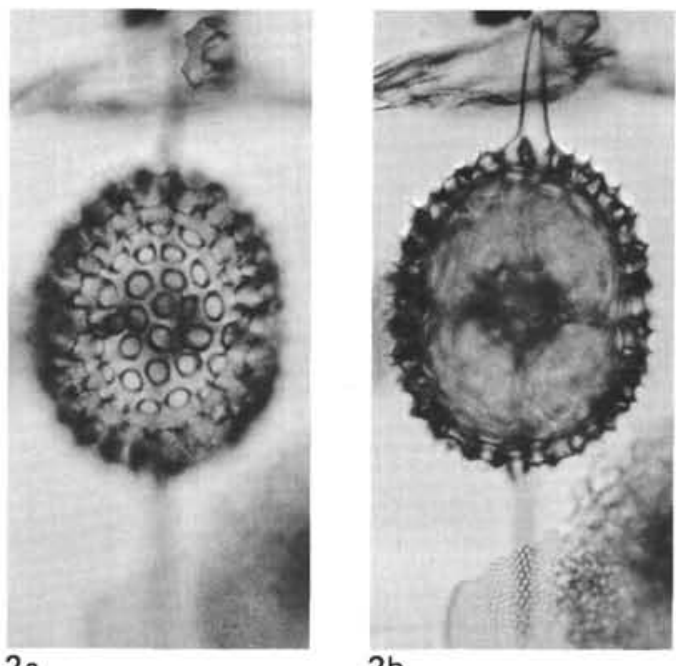

$2 b$

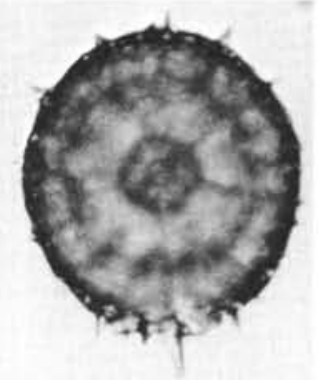

$3 c$

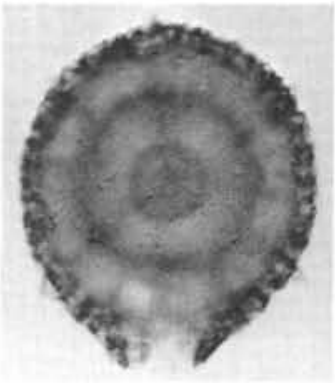

$4 c$

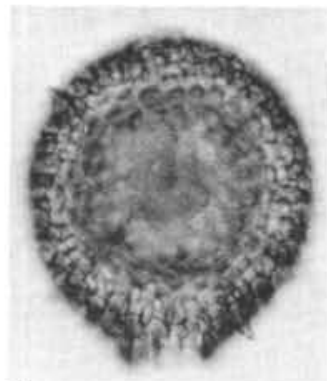

$4 b$

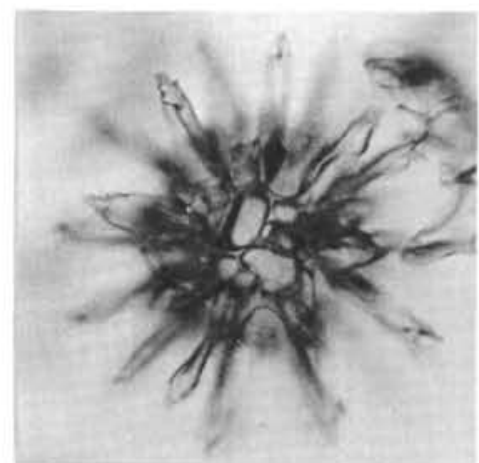

$8 a$

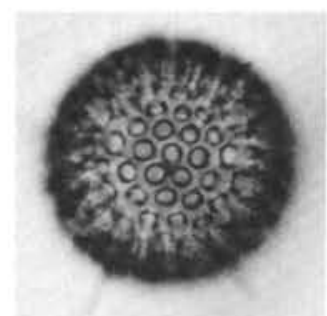

$5 a$

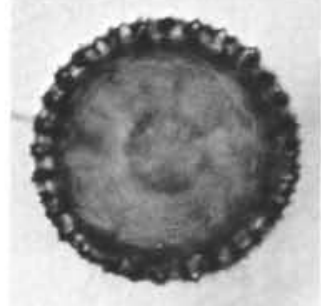

$5 b$

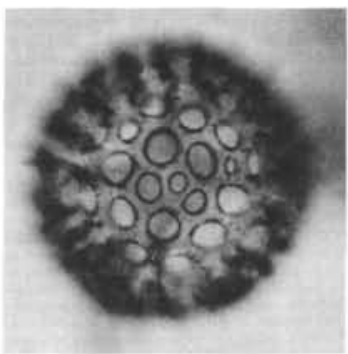

$6 a$

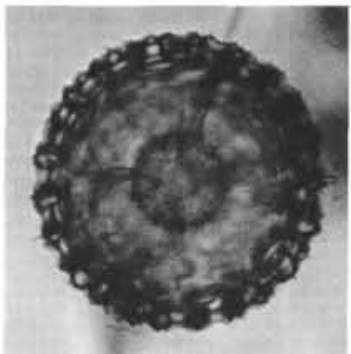

$6 b$

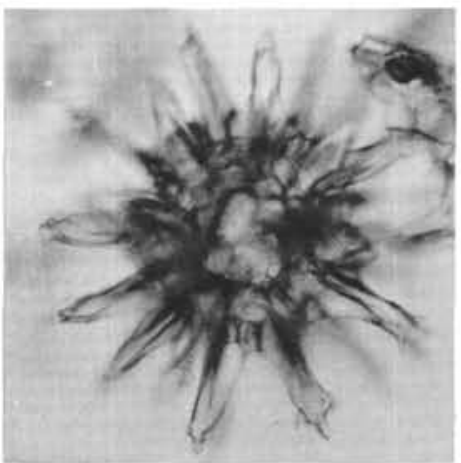

$8 b$ 


\section{PLATE 3}

All magnifications are $\times 200$.

Figures 1a, b Ommatartus tetrathalamus (Haeckel). 436-8, CC, 15-17 cm,Sl-1, N52/3.

Figures 2a, b Ommatartus avitus (Riedel).

436-22, CC, 5-7 cm, Sl-1, K45/4.

Figures 3a-5b Ommatartus penultimus (Riedel).

3. 436-30-2, 30-32 cm, Sl-1, D40/4.

4. $436-22, \mathrm{CC}, 5-7 \mathrm{~cm}, \mathrm{Sl}-1, \mathrm{M} 40 / 0$.

5. 436-31, CC, $10-12 \mathrm{~cm}, \mathrm{Sl}-1, \mathrm{H} 31 / 3$.

Figures 6a, b Ommatartus antepenultimus Riedel and Sanfilippo. 436-30-2, 30-32 cm, Sl-1, X54/2.

Figures $7 \mathrm{a}-8 \mathrm{~b}$ Cannartus laticonus Riedel.

7. 436-33, CC, 6-8 cm, Sl-1, T37/0.

8. 436-37-2, 55-57 cm, Sl-1, G43/4.

Figures 9a, b Cannartus sp. E.

436-34-2, 45-47 cm, Sl-1, T24/0.

Figures 10a, b Cannartus violina Haeckel.

436-37-4, 55-57 cm, Sl-1, V26/1.

Figures 11a, b Cannartus mammifer (Haeckel). 436-37, CC, 13-15 cm, Sl-1, Q31/1.

Figures 12a, b Cannartus sp. A.

436-36-4, 35-37 cm, Sl-1, K49/0. 
PLATE 3

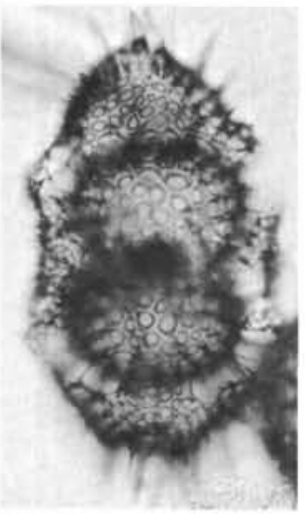

$1 a$

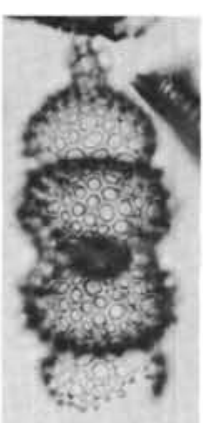

$4 a$

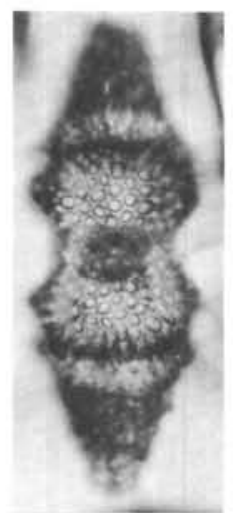

$7 a$

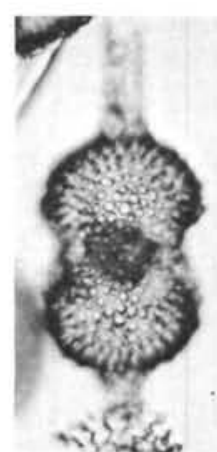

$10 \mathrm{a}$

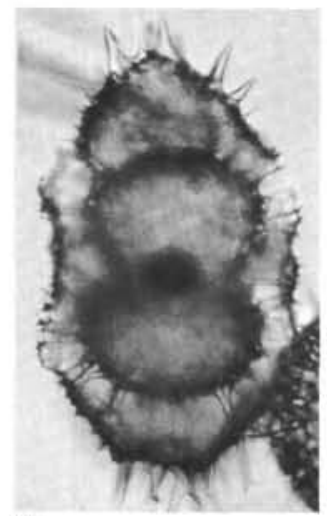

$1 \mathrm{~b}$

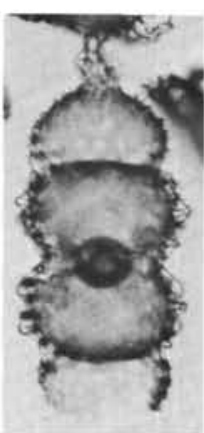

$4 b$

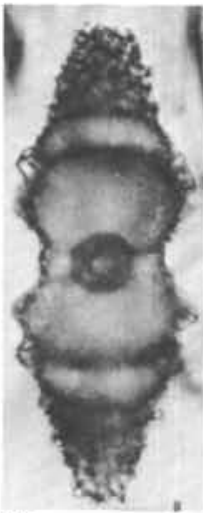

$7 b$

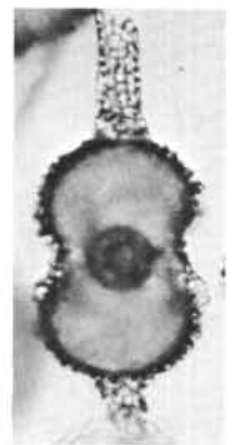

$10 \mathrm{~b}$

$8 a$

$5 a$
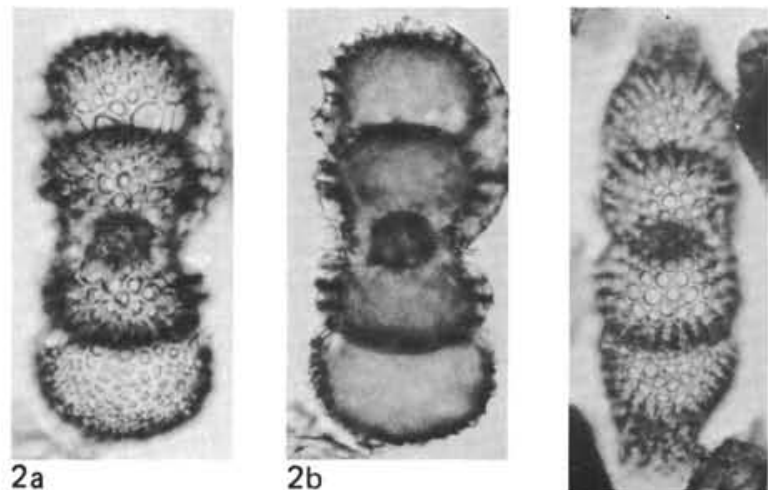

3a

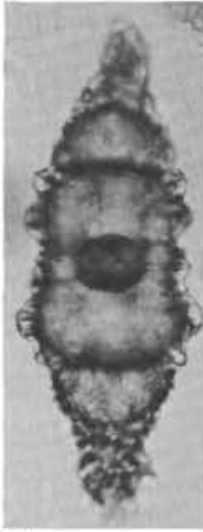

$5 b$
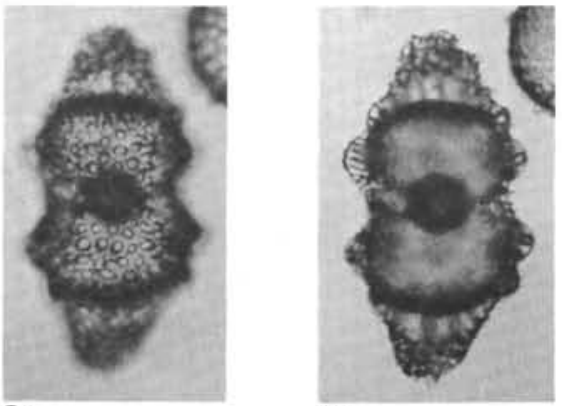

$8 b$
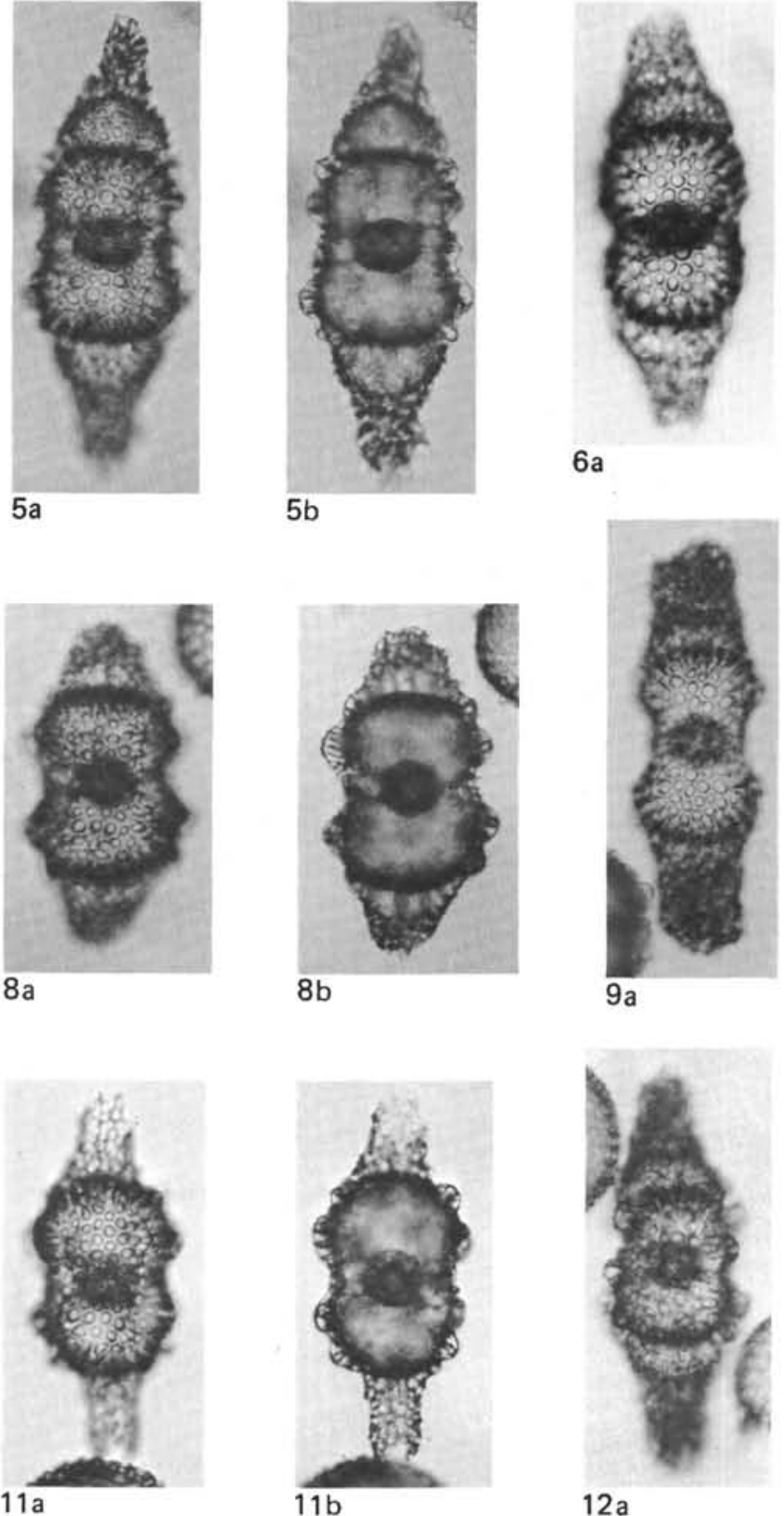

$9 a$

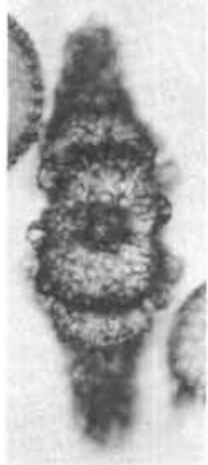

$12 a$
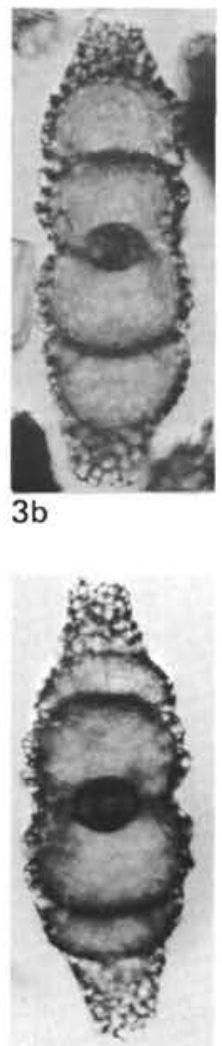

$6 b$

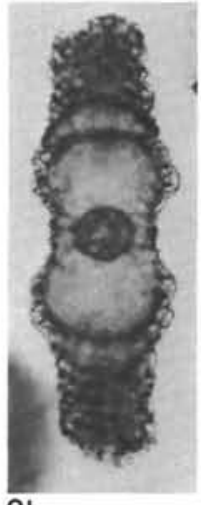

$9 b$

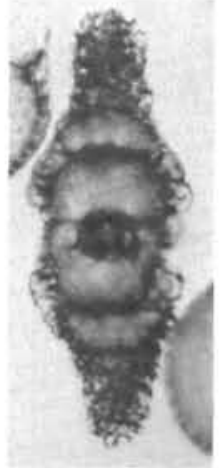

$12 b$ 
PLATE 4

All magnifications are $\times 200$.

Figure 1 Artiscinae gen. et sp. indet.

436-4-3, 27-29 cm, Sl-1, D30/3.

Figures 2a-c, Ommatartus didymus (Ehrenberg).

$5 \mathrm{a}, \mathrm{b} \quad$ 2. $436-31, \mathrm{CC}, 10-12 \mathrm{~cm}, \mathrm{Sl}-1, \mathrm{D} 36 / 1$.

Figures 3a, b Ommatartus highesi (Campbell and Clark).

436-31, CC, 10-12 cm, Sl-1, C33/2

Figures 4a, b, Cannartus petterssoni Riedel and Sanfilippo.

$6 \mathrm{a}, \mathrm{b}, 7 \mathrm{a}, \mathrm{b}$ 4. 436-33-4, 25-27 cm, Sl-1, A27/3.

6. 436-33-4, 25-27 cm, Sl-1, P59/1.

7. $436-33-4,25-27 \mathrm{~cm}, \mathrm{Sl}-1, \mathrm{U} 45 / 0$.

Figures $8 \mathrm{a}, \mathrm{b} \quad$ Ommatartus sp C.

436-33, CC, 6-8 cm, Sl-1, G41/4.

Figures 9a, b Cannartus sp. B.

436-34-3, 43-45 cm, Sl-1, T54/0.

Figures 10a- Cannartus sp. C.

11b 10. 436-34, CC, 6-8 cm, Sl-1, Q36/2.

11. $436-34, \mathrm{CC}, 6-8 \mathrm{~cm}, \mathrm{Sl}-1, \mathrm{U} 59 / 0$. 
PLATE 4

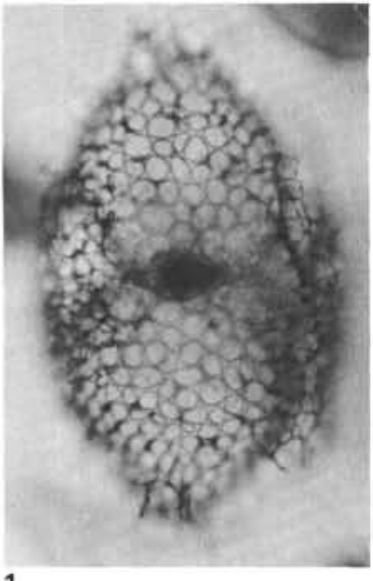

1

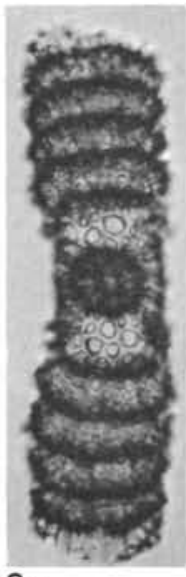

$3 a$

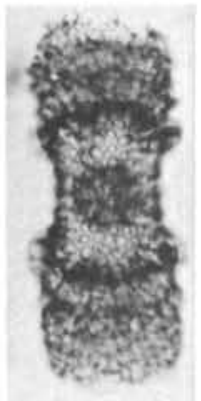

$6 a$

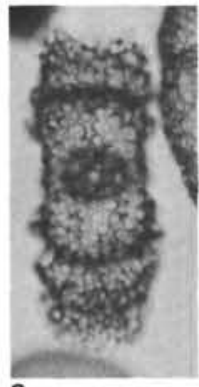

$9 a$
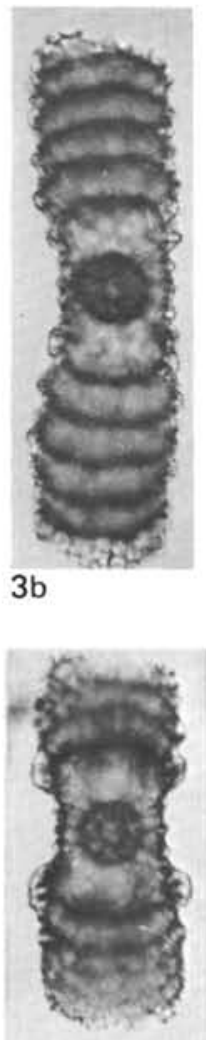

$6 b$

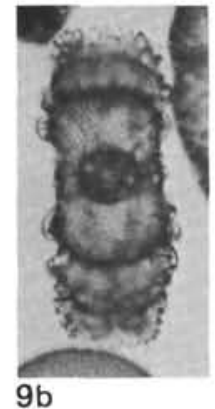

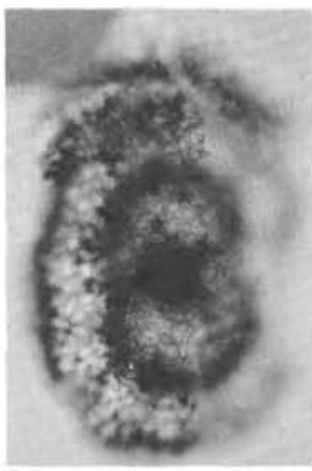

$2 a$

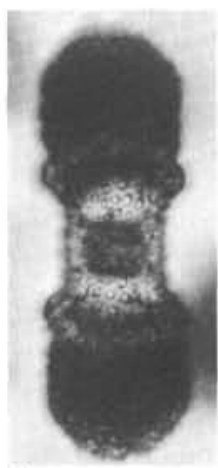

$4 a$
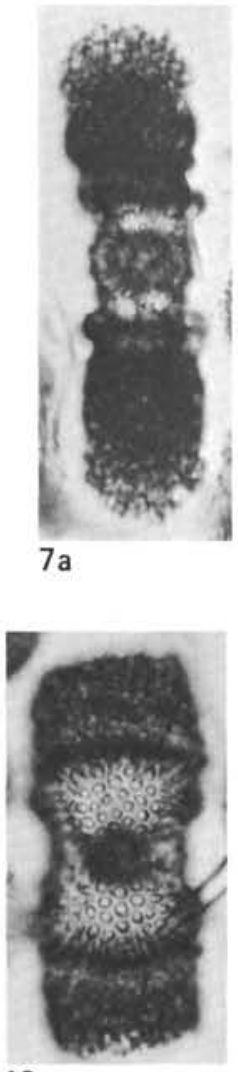

$10 \mathrm{a}$

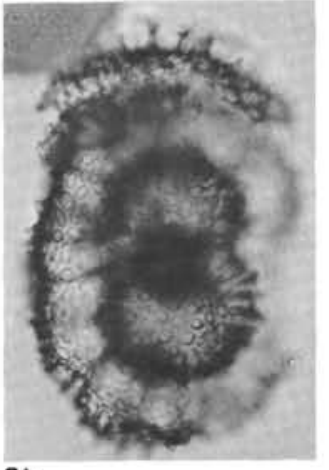

$2 b$

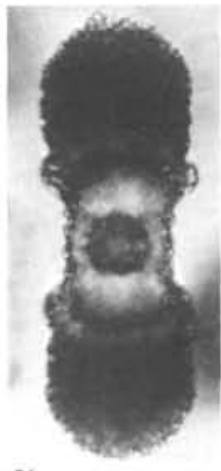

$4 b$

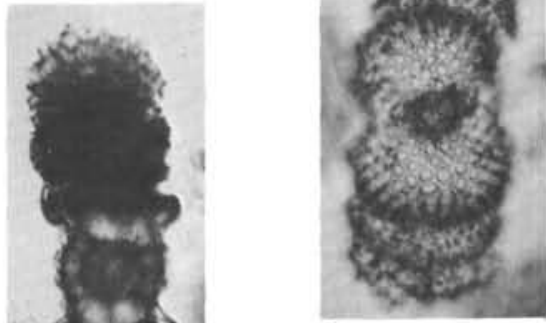

$8 a$

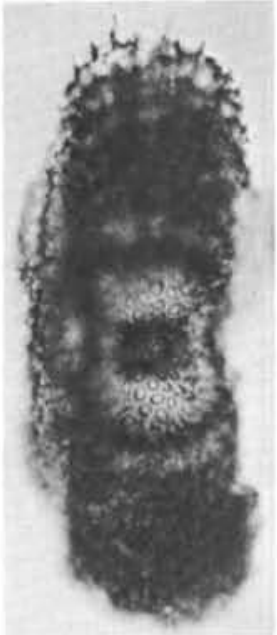

$11 \mathrm{a}$

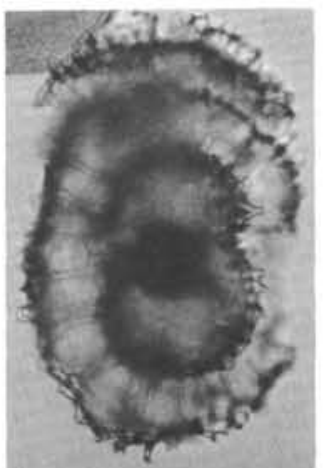

2c

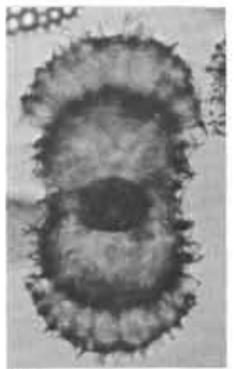

$5 b$

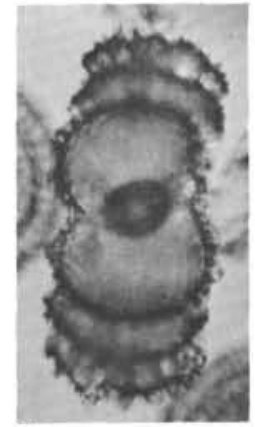

$8 b$

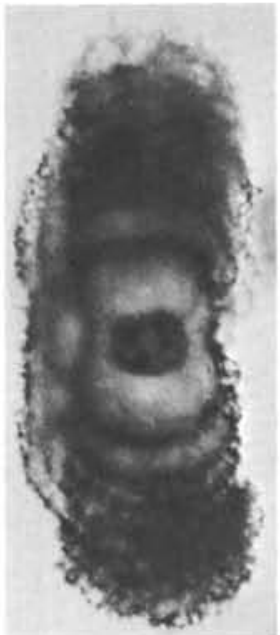

$11 \mathrm{~b}$ 
PLATE 5

All magnifications are $\times 200$.

Figures 1a, b, Ommatartus sp. D.

$4 a, b \quad$ 1. $436-26, C C, 8-10 \mathrm{~cm}, \mathrm{Sl}-1, \mathrm{~L} 46 / 1$.

4. 436-27-3, 46-48 cm, Sl-1, P55/0.

Figures 2a, b, Cannartus spp.

5a-6b 2. $436-30-2,30-32 \mathrm{~cm}, \mathrm{Sl}-1, \mathrm{C} 28 / 3$.

5. 436-35-3, 50-52 cm, Sl-1, Q $45 / 4$.

6. 436-30, CC, 9-11 cm, Sl-1, U30/0.

Figures 3a, b Ommatartus ? sp.

436-27-3, 46-48 cm, Sl-1, R36/3.

Figures 7a-10b Cannartus sp. D.

7. 436-34,CC, 6-8 cm, Sl-1, T44/4.

8. 436-35-1, 50-52 cm, Sl-1, L48/0.

9. 436-36-4, 35-37 cm, Sl-1, C35/0.

10. $436-36-7,25-27 \mathrm{~cm}, \mathrm{Sl}-1, \mathrm{~K} 64 / 0$.

Figures 11a, b Cannartus tubarius (Haeckel).

436-37, CC, 13-15 cm, Sl-1, H48/3. 
PLATE 5

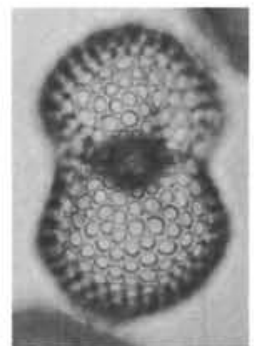

$1 a$

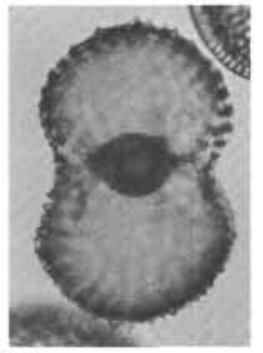

$1 b$

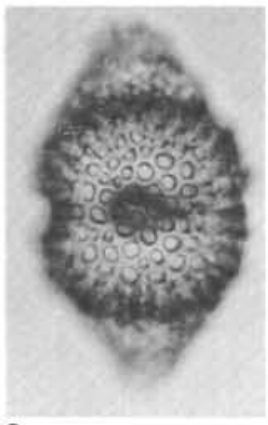

$2 a$

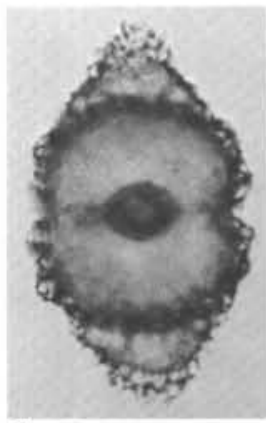

$2 b$

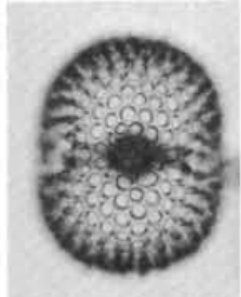

$3 a$
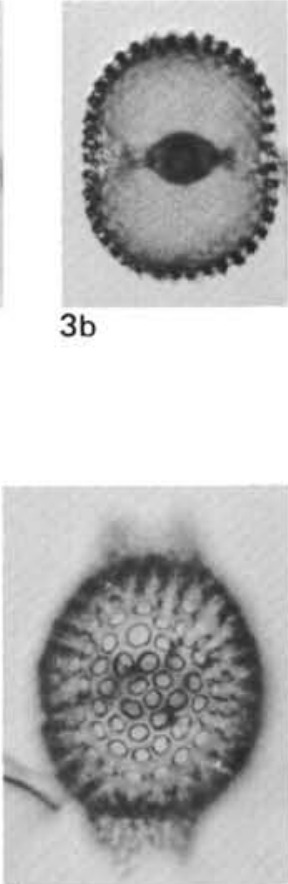

$6 a$

$4 a$

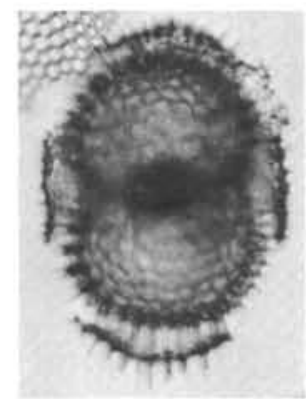

$4 b$

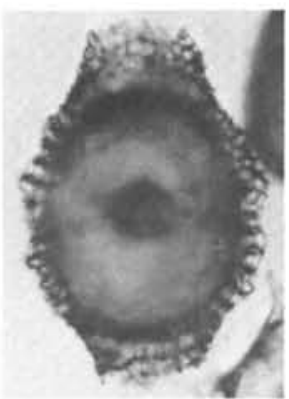

$5 a$

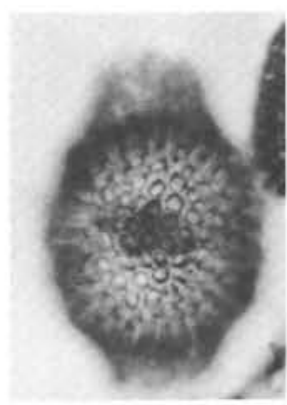

$5 b$

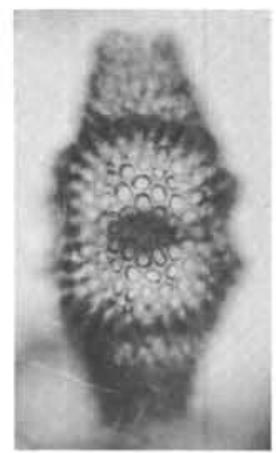

$7 a$

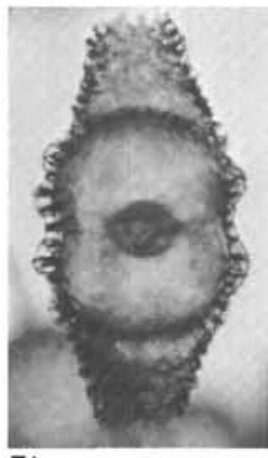

$7 b$
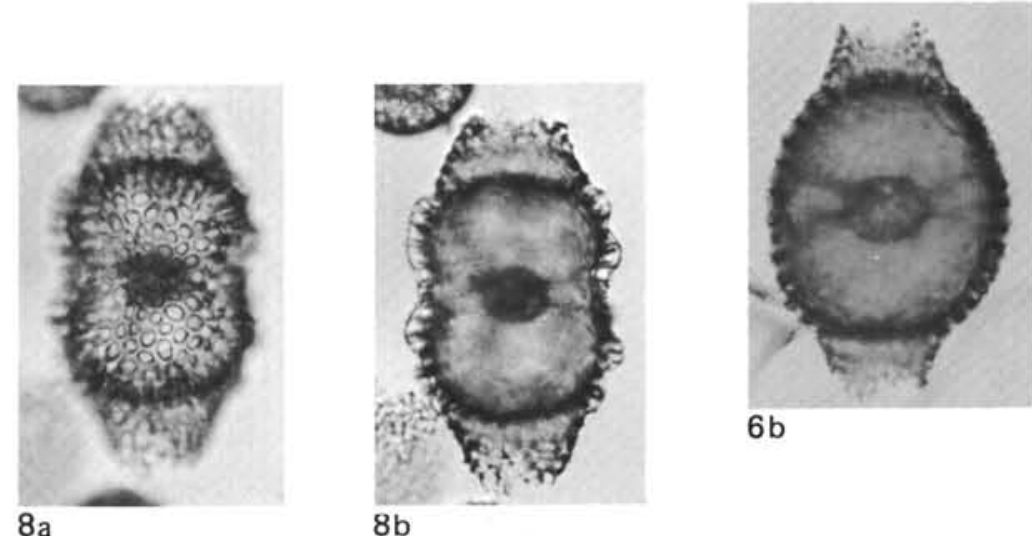

$6 b$

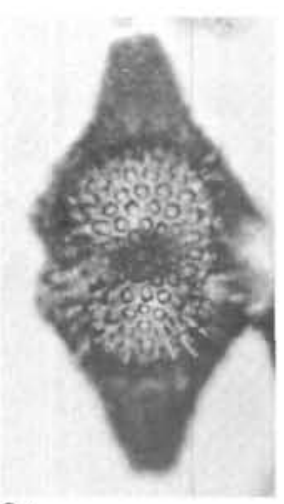

$9 a$
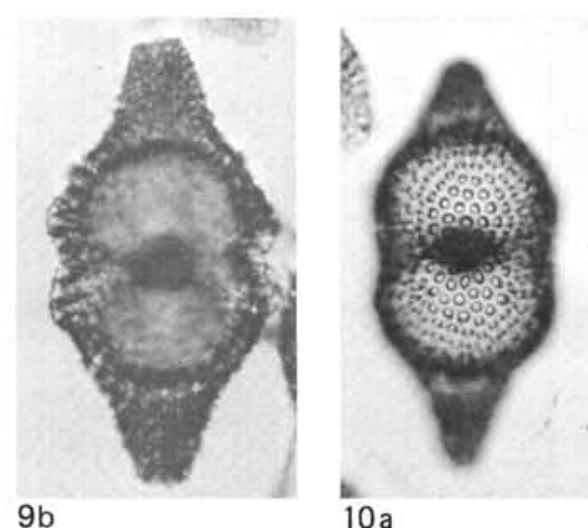

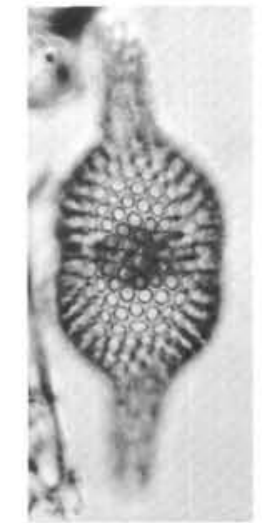

$10 \mathrm{~b}$

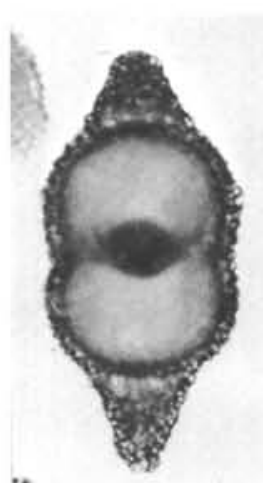

$11 \mathrm{a}$

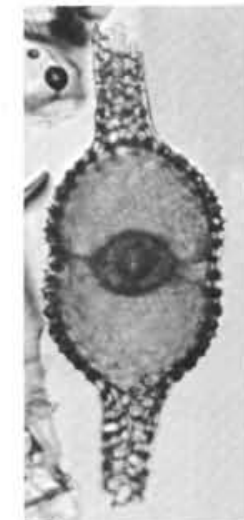

$11 \mathrm{~b}$ 


\section{PLATE 6}

Unless otherwise indicated, magnifications are $\times 200$.

Figure $1 \quad$ Spongaster tetras tetras Ehrenberg. 436-4-6, 25-27 cm, Sl-1, K50/0 ( ×155).

Figure 2 Spongaster tetras irregularis Nigrini. 435-2,CC, 12-14 cm, Sl-1, E32/0 ( $\times 155)$.

Figure 3 Spongaster berminghami (Campbell and Clark). 436-30-2, 30-32 cm, Sl-1, T59/3 ( $\times 155)$.

Figure $4 \quad$ Spongodiscus ambus Sanfilippo and Riedel. 436-22, CC, 5-7 cm, Sl-2, B35/3.

Figure $5 \quad$ Spongodiscus $\mathrm{sp}$.

436-8, CC, $15-17 \mathrm{~cm}, \mathrm{Sl}-2, \mathrm{Y} 64 / 3$ ( $\times 155)$.

Figures 6-8b Cycladophora davisiana Ehrenberg.

6. 436-1, CC, 2-3 cm, Sl-1, X43/0.

7. $436-8, \mathrm{CC}, 15-17 \mathrm{~cm}, \mathrm{Sl}-1, \mathrm{R} 59 / 1$.

8. 436-4-3, 27-29 cm, Sl-1, U30/2.

Figures 9a-11b Clathrocyclas bicornis Hays.

9. 436-4-3, 27-29 cm, Sl-1, T32/0.

10. 436-8, CC, $15-17 \mathrm{~cm}, \mathrm{Sl}-1, \mathrm{D} 55 / 1$.

11. $436-8, \mathrm{CC}, 15-17 \mathrm{~cm}, \mathrm{Sl}-1, \mathrm{~J} 54 / 0$.

Figures 12a- Clathrocyclas sp. H.

$13 \mathrm{~b} \quad 12.436-17-4,66-68 \mathrm{~cm}, \mathrm{Sl}-1, \mathrm{O} 23 / 4$.

13. 435-A-2,CC, 4-6 cm, Sl-1, T36/0.

Figure 14 Saturnalis circularis Haeckel.

436-8, CC, 15-17 cm, Sl-1, N54/4. 


\section{PLATE 6}
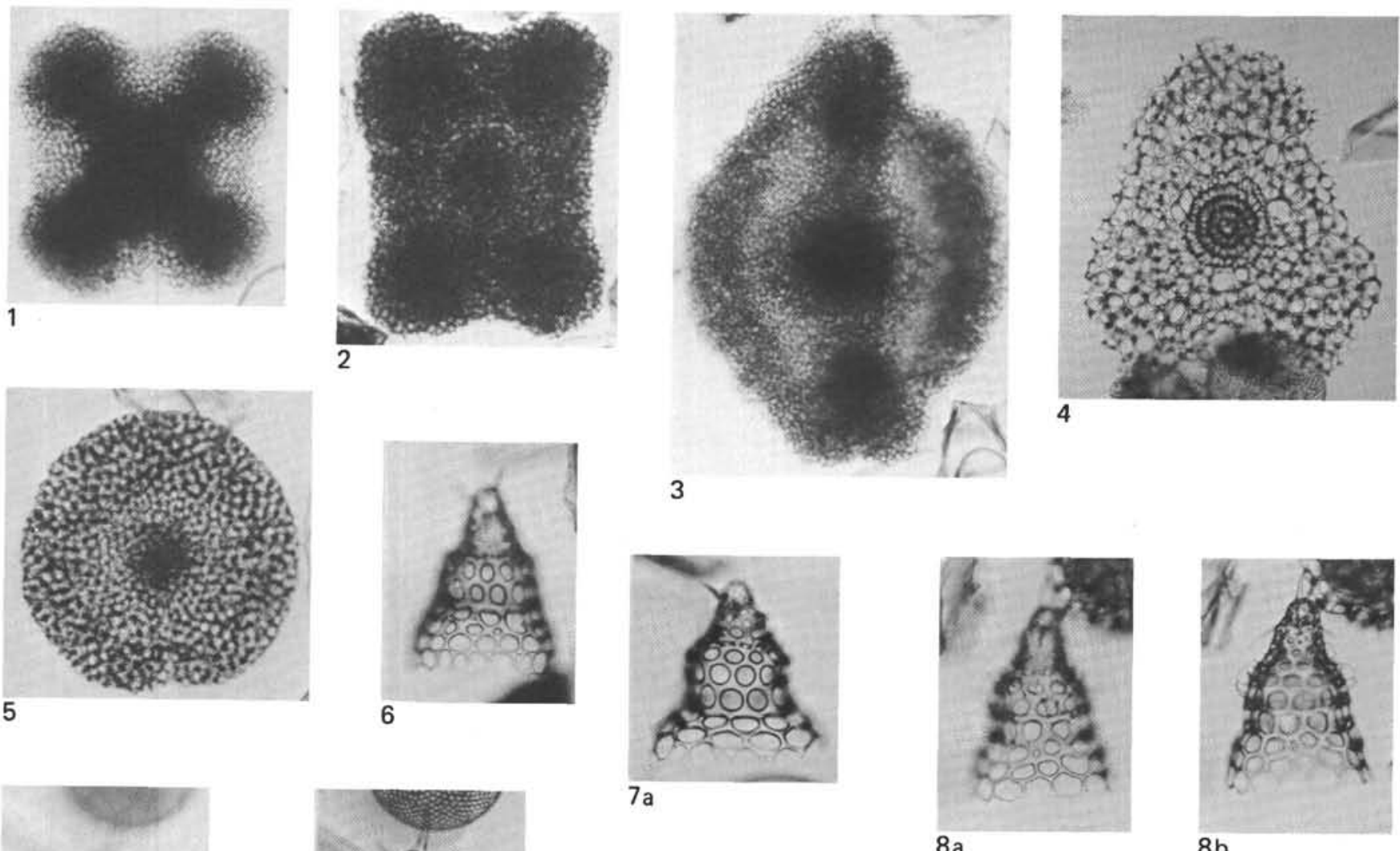

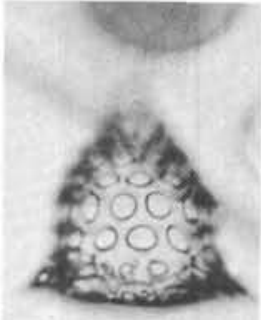

9a

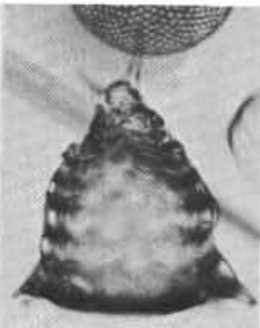

$9 b$

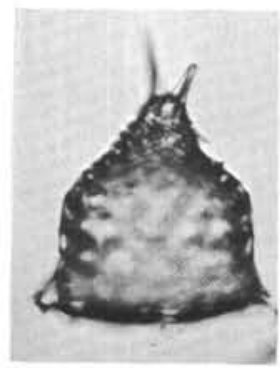

$11 \mathrm{~b}$

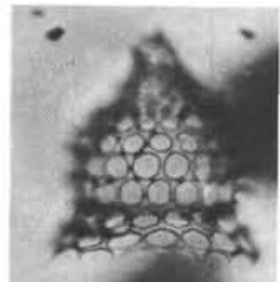

$12 \mathrm{a}$

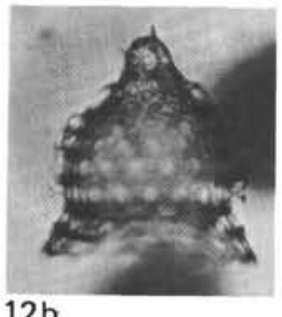

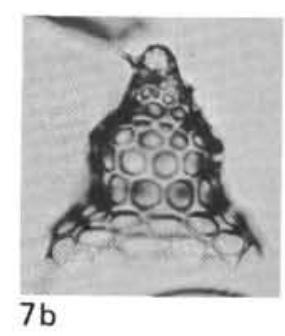
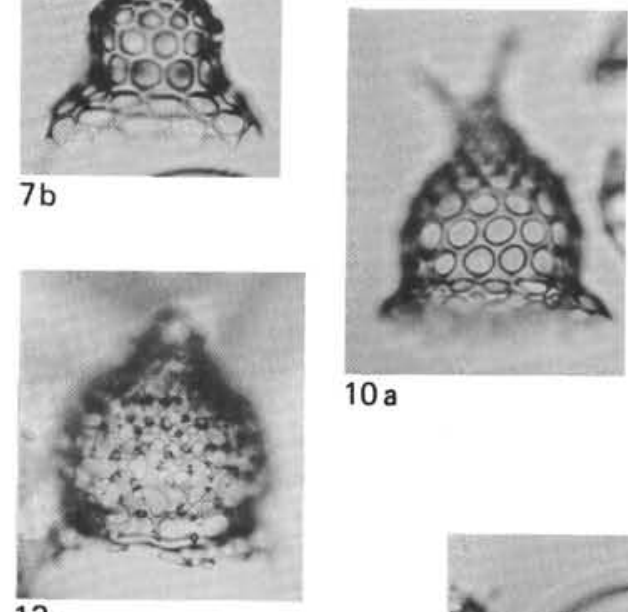

$10 \mathrm{a}$

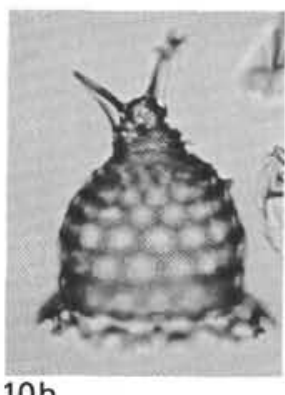

$13 a$

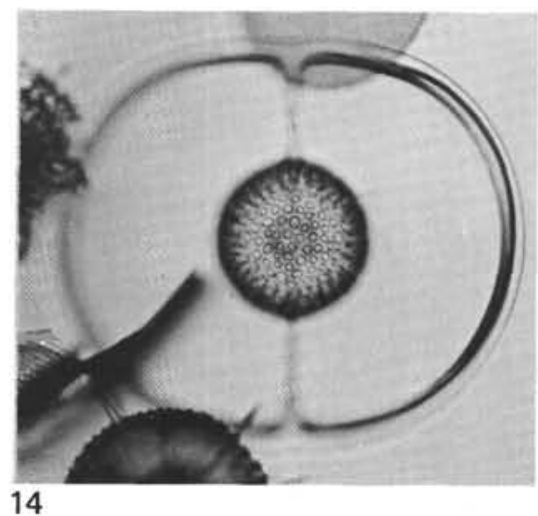




\section{PLATE 7}

All magnifications are $\times 200$.

Figures 1a, b Eucyrtidium matuyamai Hays. 436-8, CC, 15-17 cm, Sl-1, A61/4.

Figures 2a, b, Eucyrtidium calvertense Martin group. 4a-6b 2. $436-8, C C, 15-17 \mathrm{~cm}, \mathrm{Sl}-1, \mathrm{~J} 28 / 4$.

4. 436-20, CC, $10-12 \mathrm{~cm}, \mathrm{Sl}-1, \mathrm{~T} 39 / 4$.

5. 436-32, CC, $13-15 \mathrm{~cm}, \mathrm{Sl}-1, \mathrm{X} 30 / 0$.

6. $436-35, \mathrm{CC}, 8-10 \mathrm{~cm}, \mathrm{Sl}-1, \mathrm{U} 29 / 0$.

Figures 3a, b Eucyrtidium yatsuoense Nakaseko. 436-38, CC, 10-12 cm, Sl-1, D40/3.

Figures 7a, b Eucyrtidium sp. B. 436-38, CC, 10-12 cm, Sl-1, K49/1.

Figures 8a-10 Eucyrtidium cienkowskii Haeckel group.

8. 436-34,CC, 6-8 cm, Sl-1, X63/0.

9. 436-38, CC, $10-12 \mathrm{~cm}, \mathrm{Sl}-1, \mathrm{P} 65 / 0$.

10. 436-36-7, 25-27 cm, Sl-1, V55/2.

Figures 11a, b Eucyrtidium inflatum Kling. 436-36-7, 25-27 cm, Sl-1, B65/2.

Figures 12a- Eucyrtidium asanoi Sakai, n. sp. $14 \mathrm{~b} \quad 12.436-36-4,35-37 \mathrm{~cm}, \mathrm{Sl}-1, \mathrm{~J} 49 / 1$.

13. (Holotype). 436-36-4, 35-37 cm, Sl-1, J30/4. 14. $436-36-4,35-37 \mathrm{~cm}, \mathrm{Sl}-1, \mathrm{~V} 33 / 0$.

Figure $15 \quad$ Eucyrtidium sp. cf. E. cienkowskii Haeckel. 436-36-7, 25-27 cm, Sl-1, G56/2. 
PLATE 7

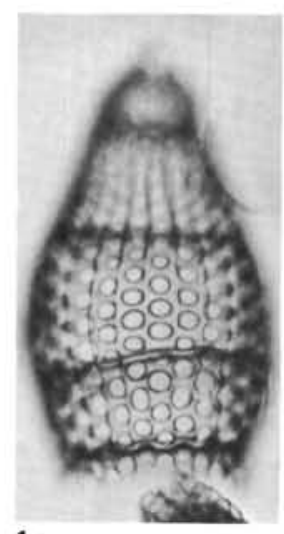

$1 \mathrm{a}$

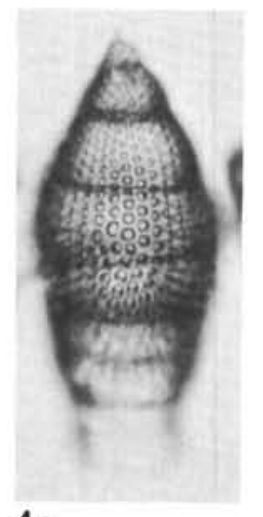

4 a

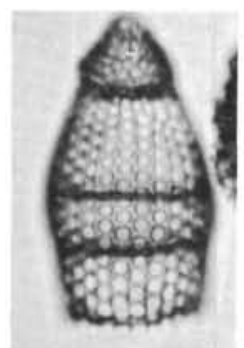

$11 \mathrm{a}$

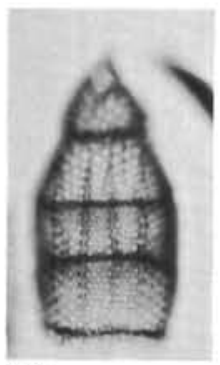

$13 \mathrm{a}$
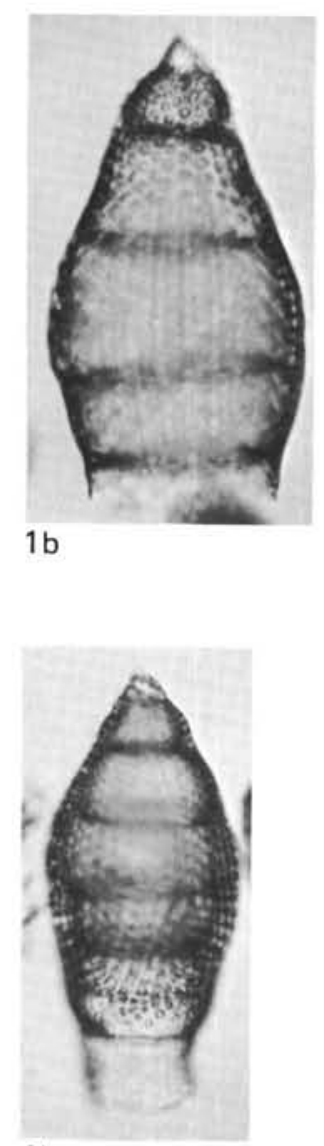

$4 b$

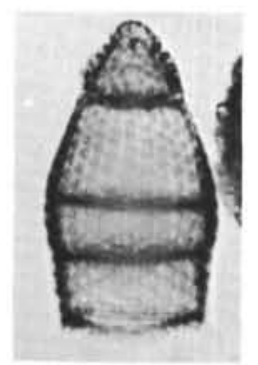

$11 \mathrm{~b}$

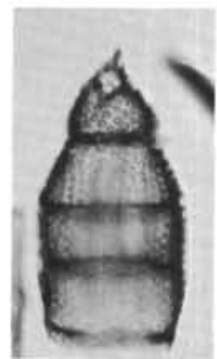

$13 b$

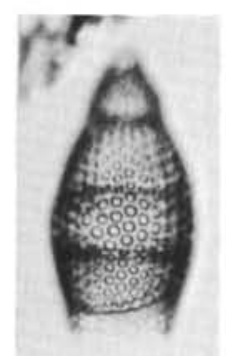

$2 a$
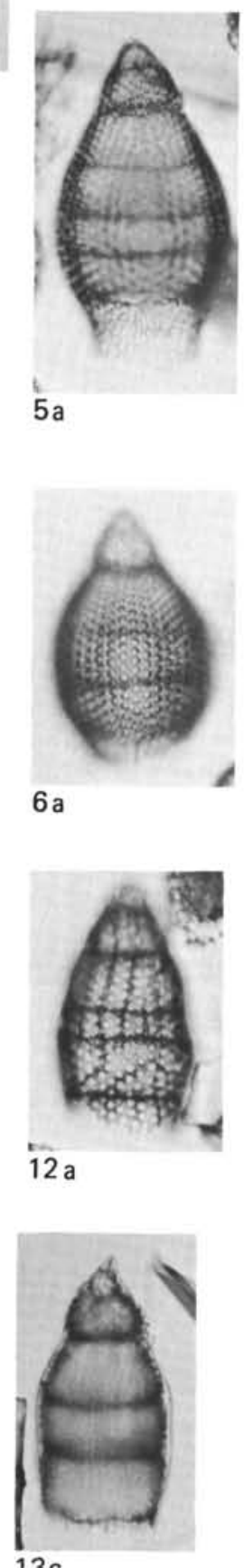

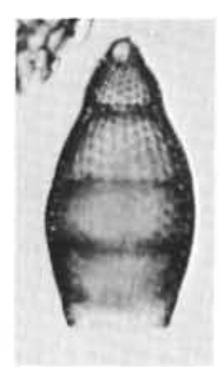

$2 b$

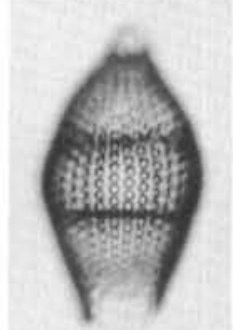

3a
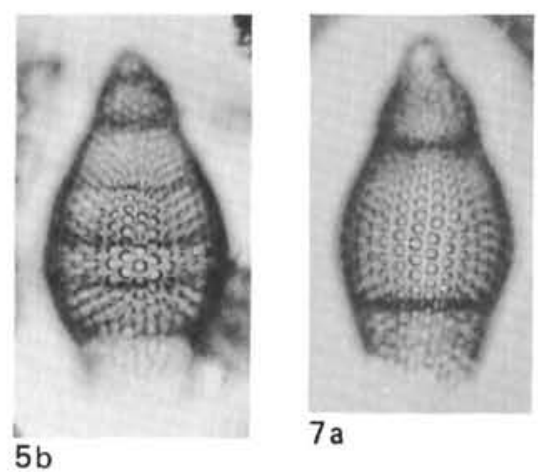

$7 a$

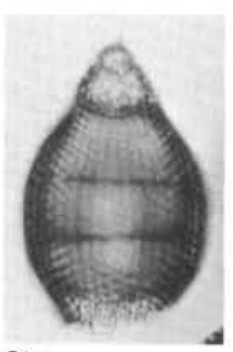

$6 b$
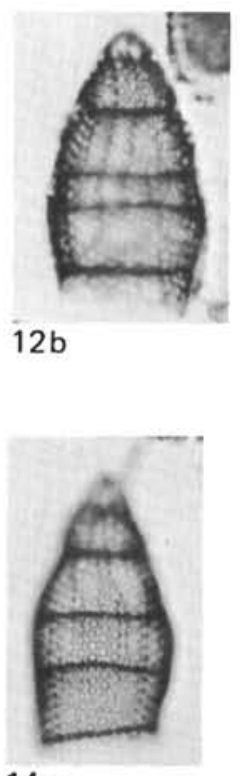

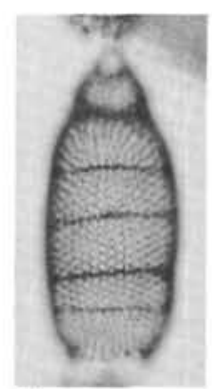

8 a

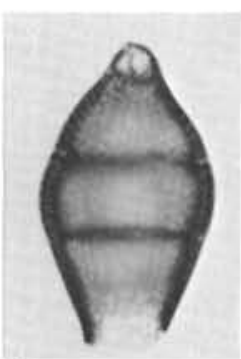

$3 b$

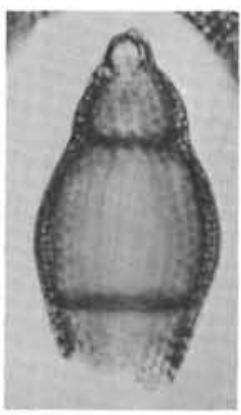

$7 b$

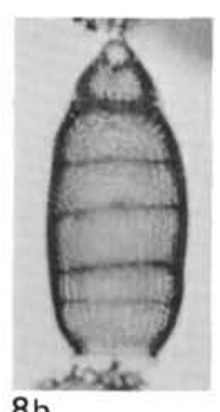

$8 b$

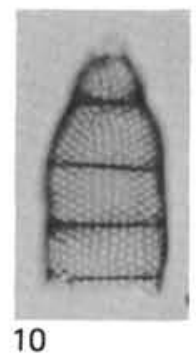

9
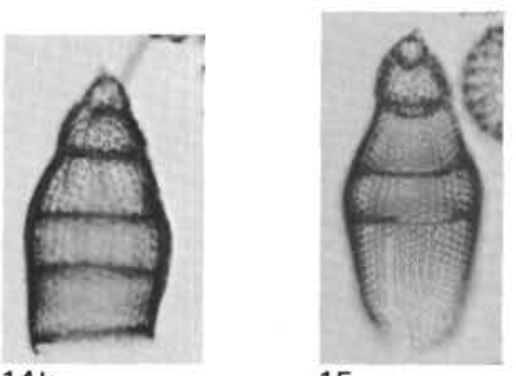
PLATE 8

All magnifications are $\times 200$.

Figures 1, 2 Stichocorys peregrina (Riedel).

1. $436-26, \mathrm{CC}, 8-10 \mathrm{~cm}, \mathrm{Sl}-1, \mathrm{~T} 33 / 4$.

2. $436-26, \mathrm{CC}, 8-10 \mathrm{~cm}, \mathrm{Sl}-1, \mathrm{Q} 28 / 0$.

Figure 3 Stichocorys delmontensis (Campbell and Clark). 436-37, CC, 13-15 cm, Sl-1, T35/4.

Figures $4 \mathrm{a}, \mathrm{b}$ Stichocorys almata (Haeckel). 436-37-2, 55-57 cm, Sl-1, E31/3.

Figures 5, 6 Cyrtocapsella tetrapera (Haeckel).

5. 436-38, CC, $10-12 \mathrm{~cm}, \mathrm{Sl}-1, \mathrm{~S} 31 / 0$.

6. 436-38, CC, 10-12 cm, Sl-1, B49/4.

Figures 7a, b Crytocapsella japonica (Nakaseko). 436-39-1, 15-17 cm, Sl-1, S42/0.

Figures 8a, b Crytocapsella cornuta (Haeckel). 436-38, CC, 10-12 cm, Sl-1, M51/4.

Figure 9 Lithopera bacca Ehrenberg. 436-10, CC, 3-5 cm, Sl-1, D27/0.

Figures 10, 11 Lithopera neotera Sanfilippo and Riedel. 10. 436-33, CC, 6-8 cm, Sl-1, G35/1. 11. $436-34-3,43-45 \mathrm{~cm}, \mathrm{Sl}-1, \mathrm{Z} 51 / 1$.

Figure 12 Lithopera renzae Sanfilippo and Riedel. 436-36-4, 35-37 cm, Sl-1, J48/0.

Figure 13 Theocorys redondoensis (Campbell and Clark). 436-31, CC, 10-12 cm, Sl-1, K49/4.

Figure 14 Theocorys spongoconum Kling. 436-38-6, 50-52 cm, Sl-1, F42/3.

Figures 15a, b Pterocanium prismatium Riedel. 436-13-5, 70-72 cm, Sl-1, F41/0.

Figures 16a, b Lychnodictum audax Riedel. 436-37-4, 55-57 cm, Sl-1, H50/1. 
PLATE 8

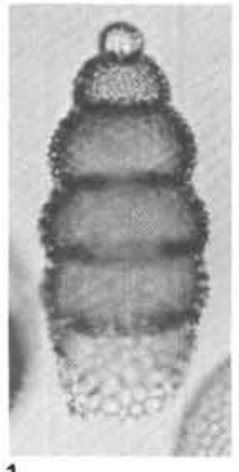

1

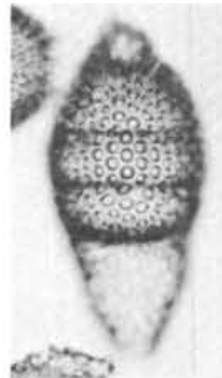

5

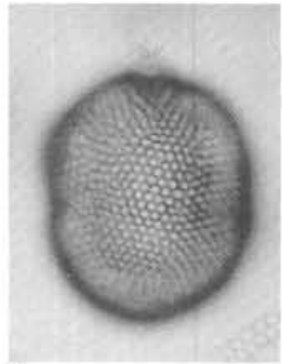

9

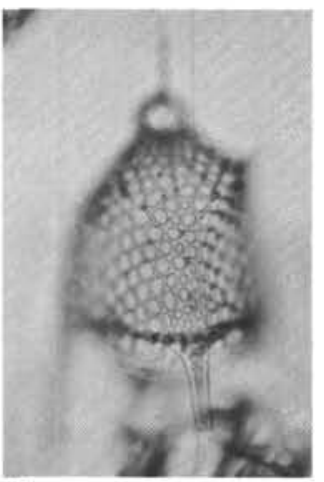

$15 a$
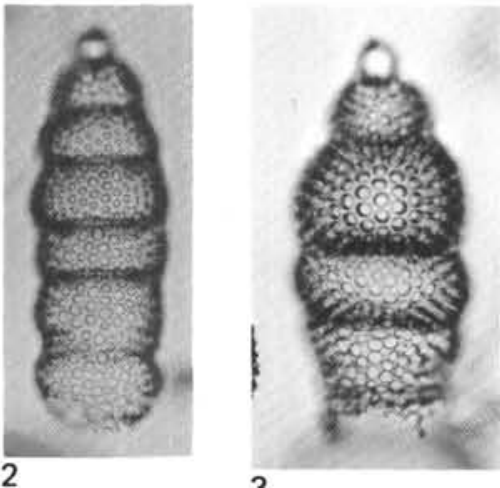

3
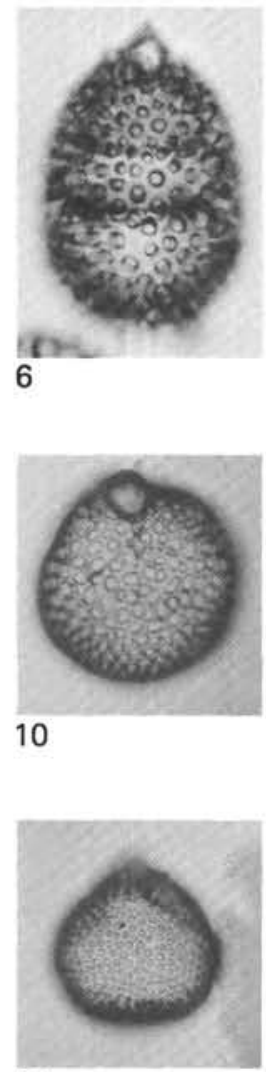

11

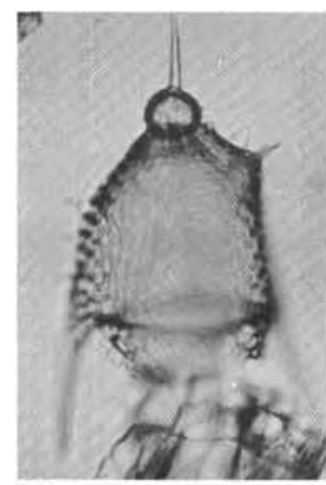

$15 b$

12
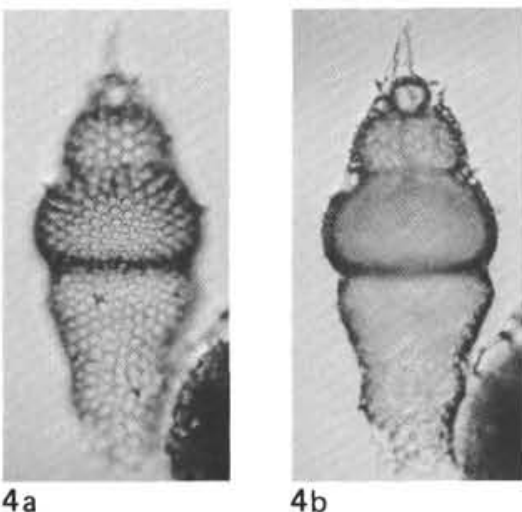

$4 b$
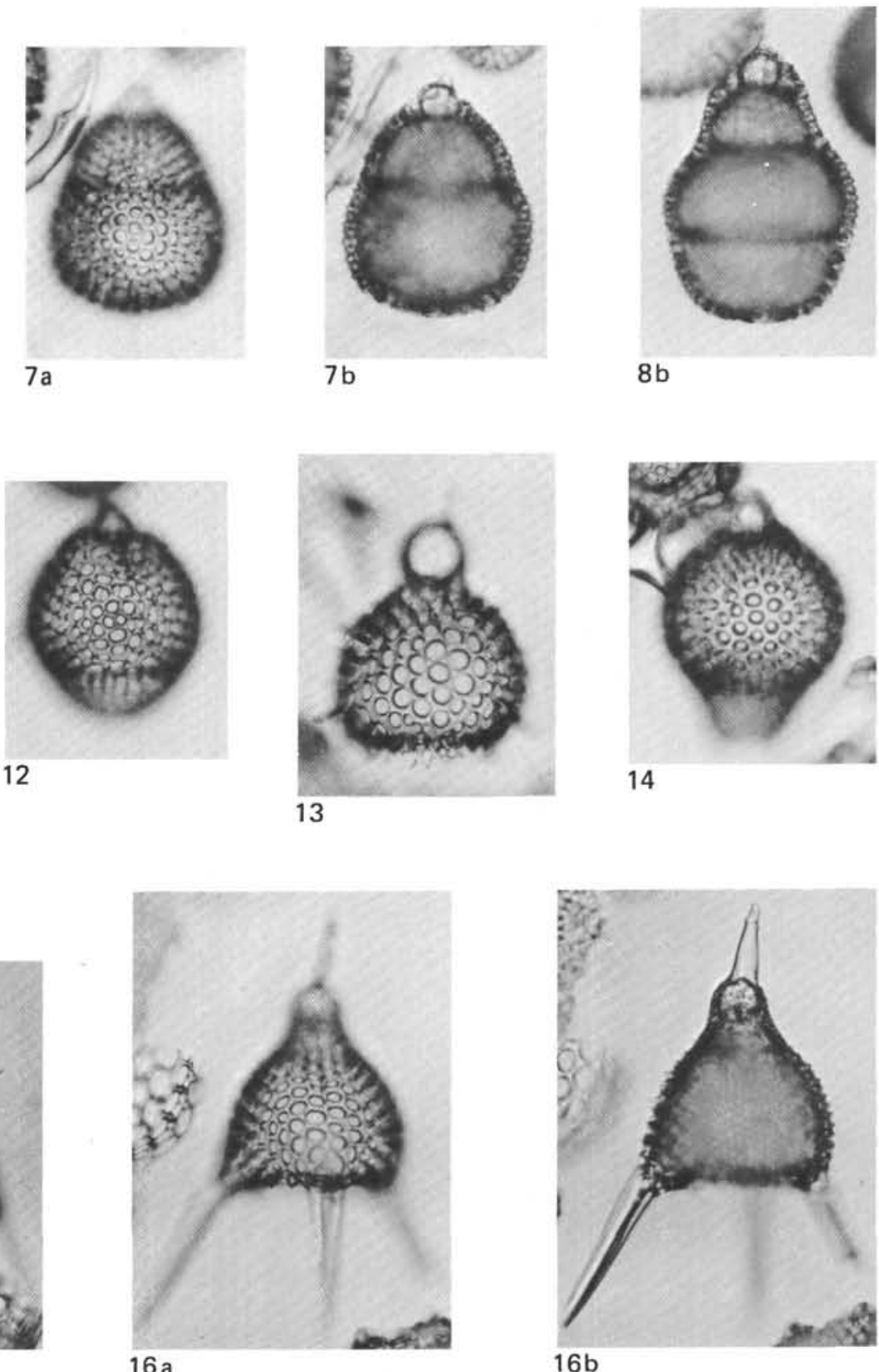
PLATE 9

Unless otherwise indicated, magnifications are $\times 200$.

Figures 1a, b Lychnocanoma sp.

436-1, CC, 2-3 cm, Sl-1, W27/0.

Figures 2a, b Lychnocanoma nipponica nipponica Nakaseko. 436-33, CC, 6-8 cm, Sl-1, P36/0.

Figures 3a, b Lychnocanoma nipponica magnacornuta Sakai, n. subsp.

436-34-2, 45-47 cm, Sl-1, H46/0.

Figure $4 \quad$ Lychnocanoma elongata (Vinassa). 436-37-4, 55-57 cm, Sl-1, E61/1 ( × 155).

Figures 5-6b Pterocorys clausus (Popofsky) group.

5. 436-4-6, 25-27 cm, Sl-1, D57/3.

6. 436-8-CC, 15-17 cm, Sl-1, F48/0.

Figure $7 \quad$ Anthocyrtidium angulare Nigrini. 436-7, CC, 7-9 cm, Sl-1, M38/2.

Figures 8a, b Lamprocyrtis haysi Kling. 435-1,CC, 5-7 cm, Sl-1, E56/1.

Figures 9a, b Lamprocyrtis neoheteroporos Kling. 434-2-1, 15-17 cm, Sl-1, Q43/0.

Figures 10-11b Lamprocyrtis heteroporos (Hays).

10. 436-13-5, 70-72 cm, Sl-2, E46/0.

11. 436-13-5, 70-72 cm, Sl-2, S63/0. 
PLATE 9

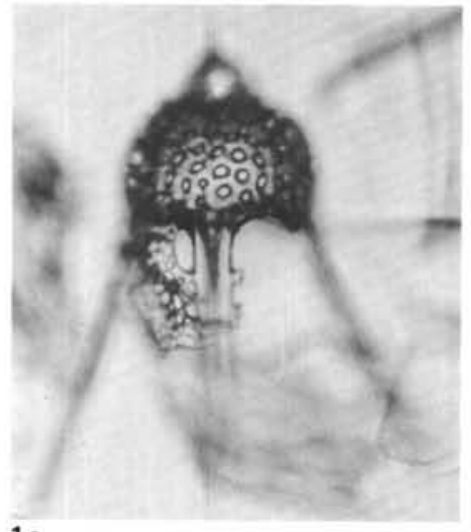

$1 \mathrm{a}$

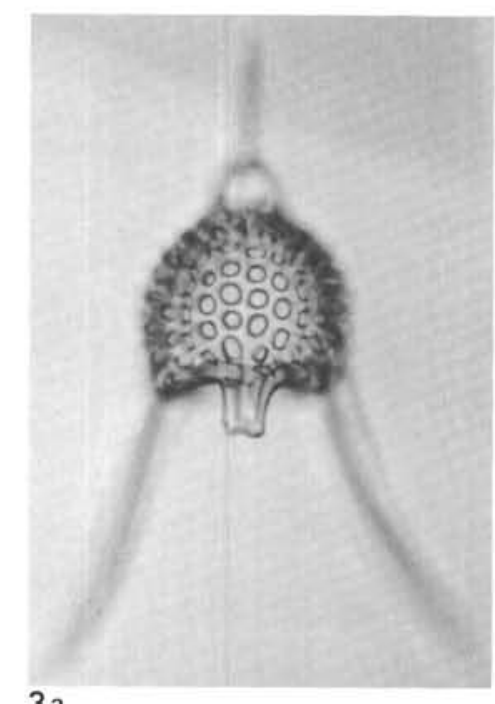

$3 a$

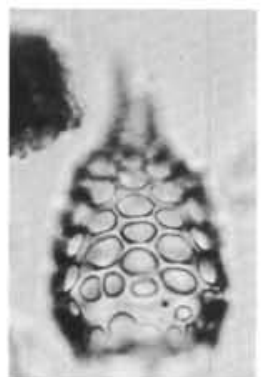

$8 a$

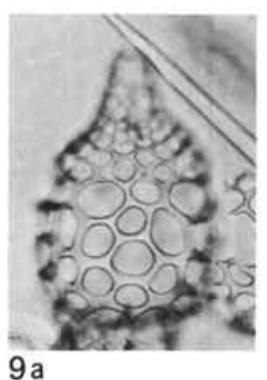

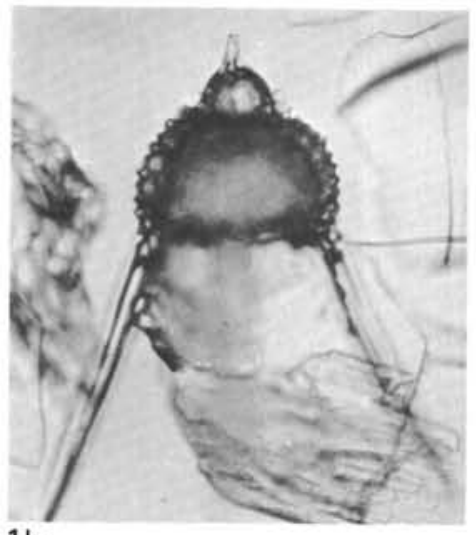

$1 \mathrm{~b}$
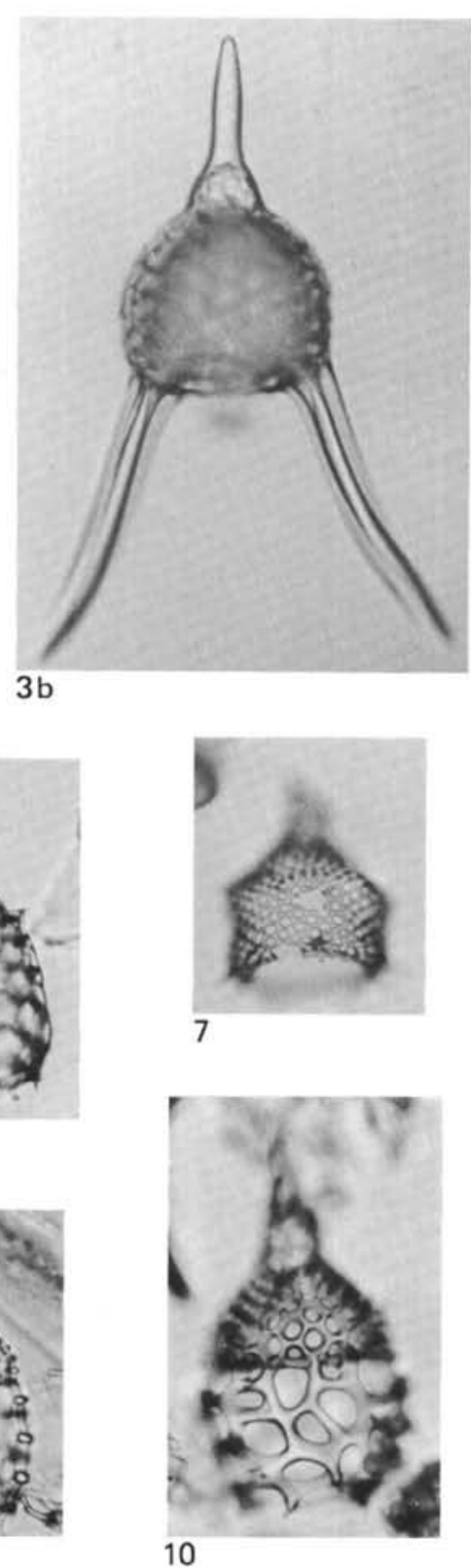
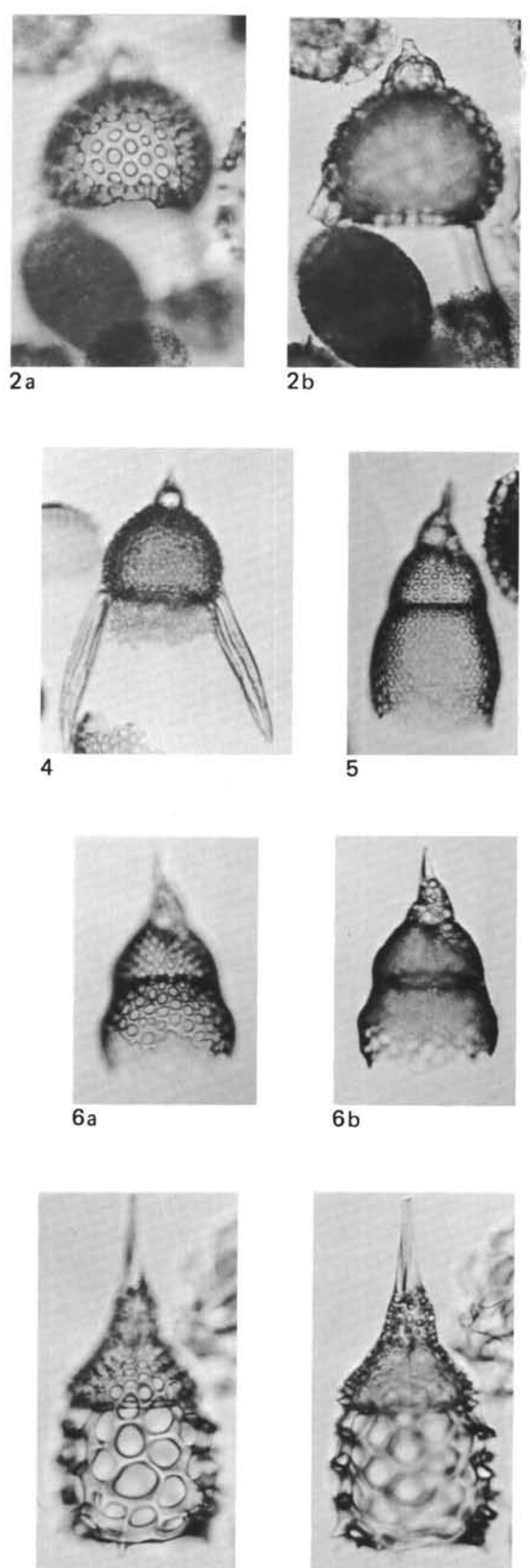

$11 \mathrm{a}$

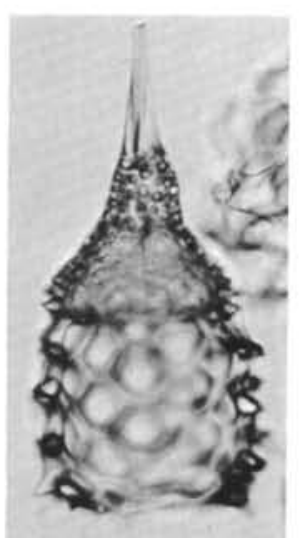

$11 \mathrm{~b}$ 
PLATE 10

Unless otherwise indicated, magnifications are $\times 200$.

Figures 1a, b Theocorythium trachelium dianae (Haeckel). 436-2-3, 51-53 cm, Sl-1, Y39/0.

Figures 2a, b Theocorythium trachelium trachelium (Ehrenberg). 436-16-7, 36-38 cm, Sl-1, S53/0.

Figures 3a, b Theocorythium vetulum Nigrini. 436-16-7, 36-38 cm, Sl-2, K25/3.

Figures $4 \mathrm{a}, \mathrm{b}$ Theocorythium sp.

436-16-7, 36-38 cm, Sl-2, B50/2.

Figures 5a, b Calocycletta virginis Haeckel. 436-38-6, 50-52 cm, Sl-1, O29/0.

Figures 6a, b Calocycletta costata (Riedel). 436-38-6, 50-52 cm, Sl-1, G38/2.

Figure $7 \quad$ Dorcadospyris alata (Riedel). 436-37, CC, 13-15 cm, Sl-1, L30/0 ( ×125).

Figure $8 \quad$ Dorcadospyris dentata Haeckel. 436-39-1, 15-17 cm, Sl-1, G51/3 (×155).

Figures 9, 10 Dorcadospyris sp. cf. D. dentata Haeckel.

9. 436-39-1, 15-17 cm, Sl-1, J60/2 ( $\times 125)$.

10. $436-39-1,15-17 \mathrm{~cm}, \mathrm{Sl}-1, \mathrm{D} 60 / 0(\times 125)$. 


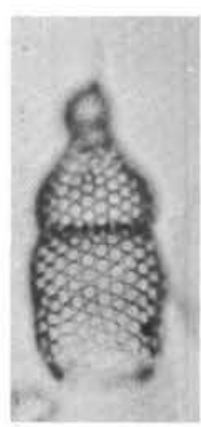

$1 \mathrm{a}$
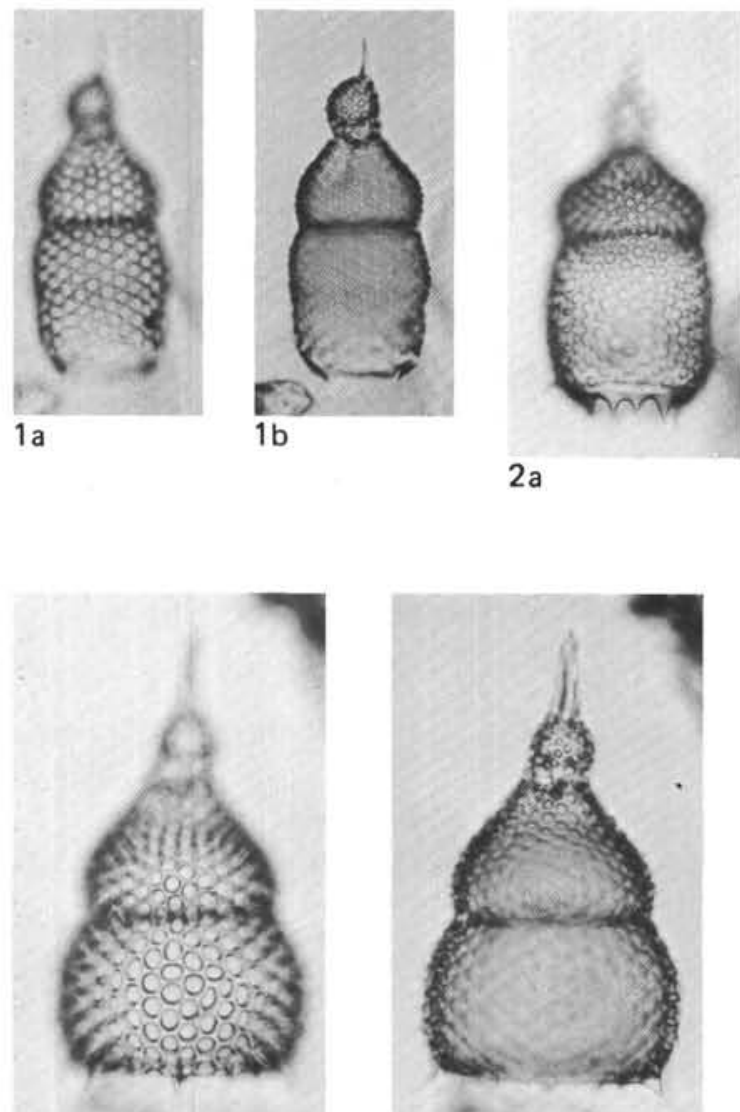

$4 \mathrm{a}$

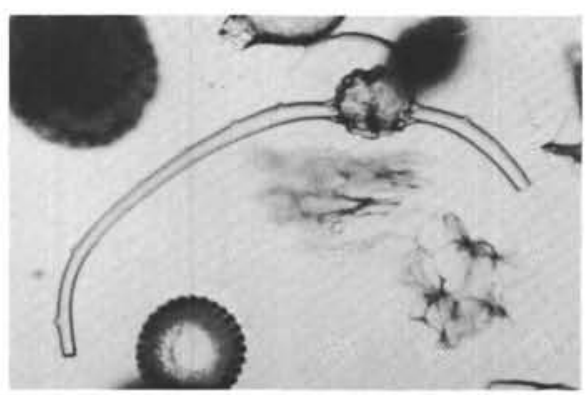

7

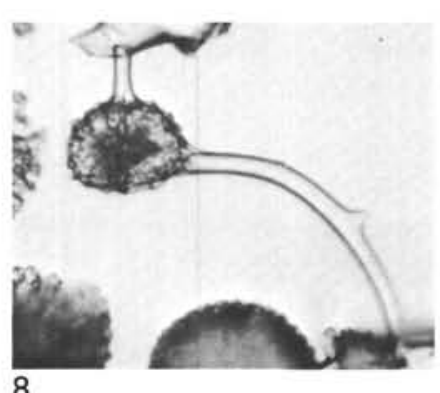

PLATE 10
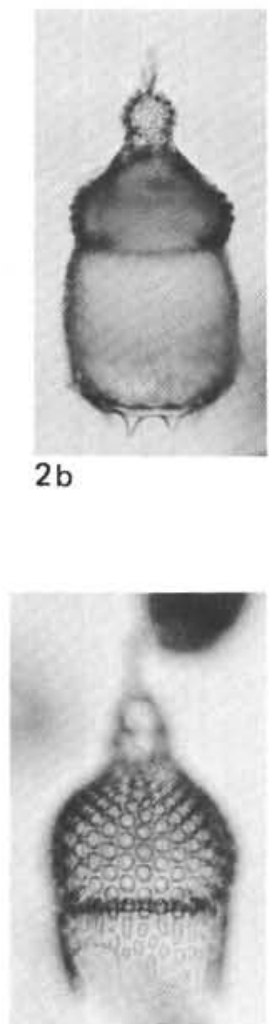

$5 a$

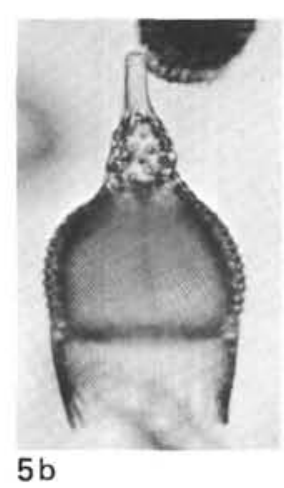

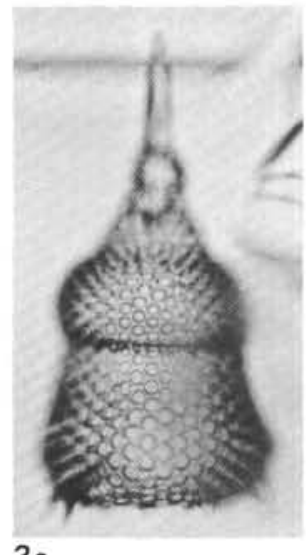

3a
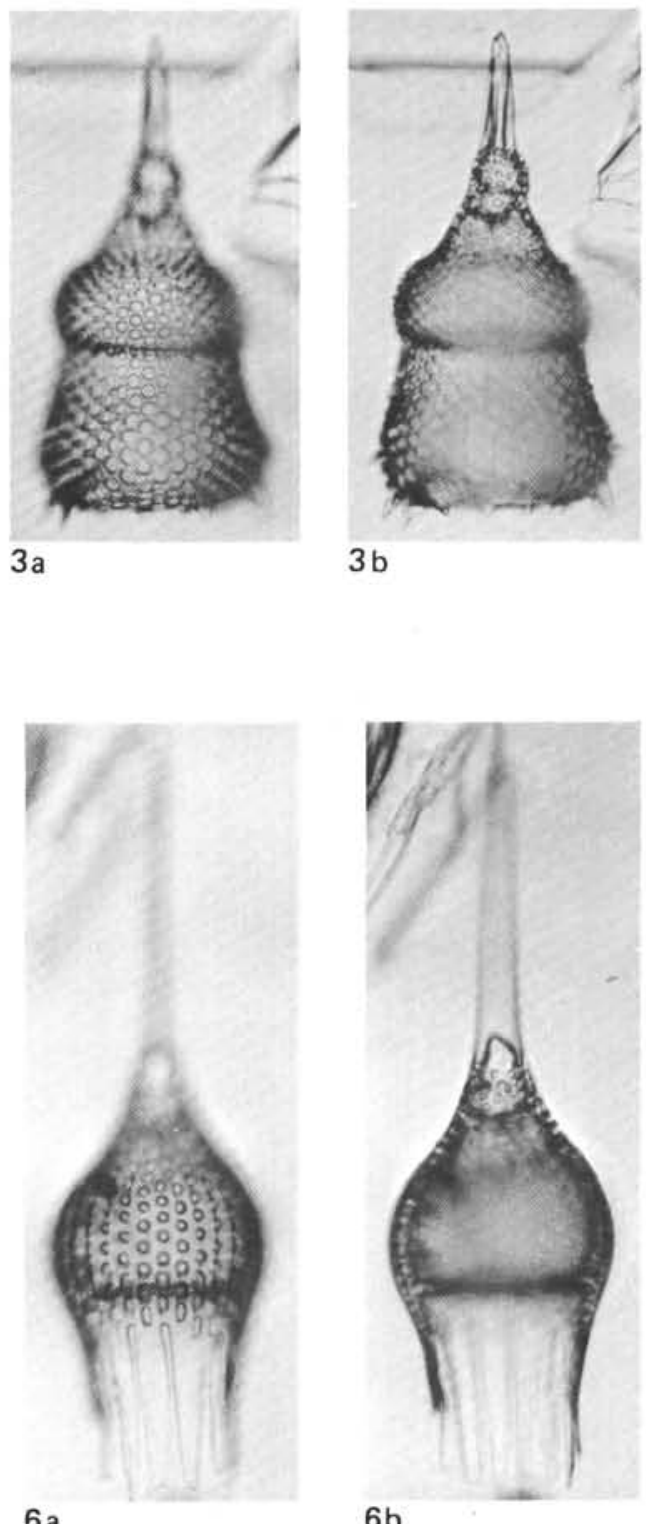

$6 b$

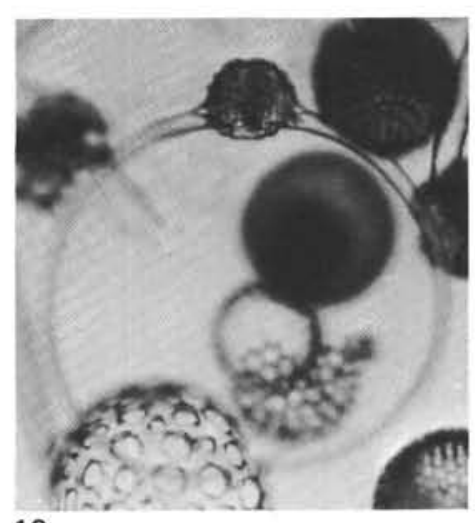

